\title{
A stratification of a moduli space of abelian varieties
}

\author{
Frans Oort
}

\section{Contents}

0 Introduction

1 Results

2 Filtrations on finite group schemes

3 Strata: the canonical stratification of the moduli space of abelian varieties

4 The Raynaud trick on $\mathcal{A}_{g} \otimes \mathbb{F}_{p}$

$5 \quad$ Filtrations on symmetric finite group schemes

6 Strata at the boundary, strata in $\mathcal{A}_{g, 1}^{*} \otimes \mathbb{F}_{p}$

7 Connecting the superspecial locus inside the supersingular locus

8 Abelian varieties with $a=g-1$

$9 \quad$ Standard types

10 Moving in a stratum

11 Moving out of a stratum

12 Transport of structure along the boundary of a stratum

13 Proof of the results

14 Some questions

15 Appendix. Some notations

References

\section{Introduction}

In this paper we study the moduli space $\mathcal{A}_{g} \otimes \mathbb{F}_{p}$ of polarized abelian varieties of dimension $g$ in positive characteristic. We construct a stratification of this space. The strata are indexed by isomorphism classes of group schemes killed by $p$; a polarized abelian variety $(X, \lambda)$ has its moduli point in a certain stratum if $X[p]$ belongs to the isomorphism class given by a certain discrete invariant. We define these invariants by a numerical property of a filtration of $N=X[p]$.

Passing from one stratum to a stratum in its boundary feels like "degenerating the $p$-structure". The fact that these strata are all quasi-affine allows us to keep going in this process until we arrive at the unique zero-dimensional stratum, the superspecial locus. One can formulate this idea by saying that the ordinary 
locus has several "boundaries", one where the abelian variety degenerates, one where the $p$-structure "becomes more special" (and an analogous idea for all non-zero-dimensional strata). This phenomenon, non-present in this form in characteristic zero, but available and powerful in positive characteristic, is expected to have many applications.

We feel that the strata $S_{\varphi}$ and the closed subsets they define inside $\mathcal{A}_{g, 1} \otimes$ $\mathbb{E}_{p}$ merit further study, see [11], where another description for our strata is given and where the cycle classes of the closures of the strata are computed. This is a natural way of producing Chow classes, or cohomology classes on $\mathcal{A}_{g, 1}$ which have a geometric interpretation when going over to characteristic $p$. It is clear that these classes depend (on $\varphi$ but also) on the characteristic $p$ chosen. Further study might show how useful they are.

Remark. A polarization $\lambda: X \rightarrow X^{t}$ on an abelian variety $X$ induces a homomorphism $\zeta: N \rightarrow N^{D}$ on $N:=X[p]$. The morphism $\lambda$ is symmetric with respect to the duality $X \mapsto X^{t}$, and $\zeta$ is anti-symmetric with respect to the duality $N \mapsto N^{D}$. Going back from $\zeta$ to some $\lambda$, and following these and their relation under deformation theory is possible, and in fact easy, if the characteristic of the base field is $\neq 2$. However, in case of $p=2$, then $+\zeta=-\zeta$ on $N=X[2]$, and in general, there are (anti-)symmetric morphisms $N \rightarrow N^{D}$ which do not come from a polarization. This causes technical complications. Sections 9 and 12 can be simplified considerably under the extra assumption $p>2$; we have chosen to treat all cases uniformly. For a proof of (9.4) in case $p>2$, see Moonen, [33]. For a proof of (12.5) in case $p>2$, see Wedhorn, [61].

Sections 2 and 4 follow closely the classification in [27]; as we need also a description of the form on $X[p]$ obtained from a principal polarization on $X$ we describe "standard types" in Section 9; this gives a classification of group schemes annihilated by $p$ with an alternating form on their Dieudonné modules over an algebraically closed field.

A short survey of the paper. The basic idea of the paper is: over an algebraically closed field there are only a finite number of finite group schemes annihilated by $p$ of a given rank. Clearly this gives subsets of $\mathcal{A}$ by considering for every abelian variety $X$ the group scheme $X[p]$. We prove, using an adaptation of an idea by Raynaud, that these strata are quasi-affine: Sections $1-4$.

Using a principal polarization on $X$ we can make this more precise, and study these strata also at the boundary, Sections $5-6$.

There is exactly one stratum of dimension one. This is contained in the supersingular locus; purely algebraic arguments show that the closure of this stratum is connected: Sections $7-8$.

Then we study in which way strata fit together. To this end we use deformation theory, in the disguise of "displays", see (15.6); we study how we can move inside a stratum, how we can move transversally out of a stratum, and 
we show that strata fit together as is required in a stratification: Sections 10 - 12. In order to be able to describe these deformations we show that every "polarized $\mathrm{BT}_{1}$ " over an algebraically closed field can be put in standard form, see Section 9; this proves the number of strata is finite.

Logical order in some arguments in this paper:

- an idea by Raynaud, generalized to our strata, proves that these strata in $\mathcal{A}^{*}$ are quasi-affine, see (6.5); using boundary behavior of strata, see (6.3), this shows that positive dimensional strata in $\mathcal{A}$ contain in their closure points of another stratum;

- using standard types we show that the boundary of a stratum is a union of lower dimensional strata, see (9.4) and (12.5);

- in Section 8 we describe the unique one-dimensional stratum and in Section 7 we show it is connected; hence all positive dimensional strata are connected.

Remark. These methods use techniques in positive characteristic. One of the corollaries of the main result of this paper is: Let $k$ be a field, fix a positive integer $n$ not divisible by char $(k)$ and consider the moduli space $\mathcal{A}_{g, 1, n} \otimes k$ of principally polarized abelian varieties of dimension $g$ with a symplectic level- $n$ structure defined over a field containing $k$; this space is irreducible.

This result is well-known: for fields of characteristic zero this is classical. For fields of positive characteristic this was proved by C.-L. Chai and by G. Faltings around 1985. Their proof followed the same line as set out by ZariskiGrothendieck-Deligne-Mumford for the moduli space of algebraic curves: first prove the result in characteristic zero, then construct "good" compactifications of moduli spaces in all characteristics, i.e. "over" Spec( $\mathbb{Z})$; then conclude by Zariski's connectedness theorem that the compactified moduli scheme in positive characteristic is connected, and derive the result. In this paper we prove the irreducibility of $\mathcal{A}_{g, 1, n} \otimes k$ for fields of positive characteristic, and hence it also shows the irreducibility in characteristic zero; as all constructions are algebraic (also the construction of the minimal compactification $\mathcal{A}^{*}$ is), this gives an algebraic proof of this fact.

The research for this paper started as joint work with Torsten Ekedahl. In an early stage we had an idea how to apply the "Raynaud trick" to non-ordinary strata; this is described in Section 4. The construction of $L$, as in Section 7 , and the proof it is connected is due to Ekedahl. Several technical details still had to be supplied; in particular the definition of the stratification, and methods like "standard types" became clear much later. Finally we decided that this author would publish the results. - I thank Torsten Ekedahl for sharing his ideas with me; the first idea of the paper is joint work, but I take full responsibility for correctness 
of the final result. - I apologize to several authors, who used already this structure, e.g. see [2], see [61], and [13], for the delay in publication of this paper.

Acknowledgments: I thank Johan de Jong, who made a suggestion which simplified the original definition of the stratification. I am grateful to Ben Moonen who spotted a mistake in an earlier version, who had an essential suggestion in one of our many stimulating discussions, and who contributed in writing down results in Section 7 . I thank Fabrizio Andreatta for useful suggestions. I thank Ching-Li Chai, Eyal Goren, Ke-Zheng Li, and the referee for helpful critical remarks.

Notations. We fix a prime number $p$. All base fields, all base schemes will be in characteristic $p$. All group schemes considered will be commutative. Polarizations on abelian varieties are supposed to be principal polarizations starting from Section 5.

For $n \in \mathbb{Z}_{>0}$ we consider the ring $\mathbb{Z}\left[\zeta_{n}, 1 / n\right]$, where $\zeta_{n}$ is a choice of a primitive $n$-th root of unity. If $p$ is a prime number and $n \in \mathbb{Z}_{>0}$ not divisible by $p$, we write $\mathbb{F}_{p, n}$ for the smallest field containing $\mathbb{F}_{p}$ and containing (a fixed choice of) a primitive $n$-th root of unity. We write $\mathcal{A}_{g, d, n} \rightarrow \operatorname{Spec}\left(\mathbb{Z}\left[\zeta_{n}, 1 / n\right]\right)$ for the moduli scheme of abelian varieties with a polarization of degree $d^{2}$ and a symplectic level- $n$-structure. When $p$ and $n$ are chosen, typically, we write $\mathcal{A}=\mathcal{A}_{g, 1, n} \otimes \mathbb{F}_{p, n}$.

\section{Results}

For every elementary sequence $\varphi$, see (5.6), there is a locally closed subset $S_{\varphi} \subset \mathcal{A}=\mathcal{A}_{g, 1, n} \otimes \mathbb{F}_{p, n}$. The unique zero-dimensional stratum $\Sigma:=S_{\{0, \ldots, 0\}}$ is closed. A point $[(X, \lambda)]$ is in $\Sigma$ iff it is superspecial, i.e. for an algebraically closed field $k$, we have $X \otimes k \cong E^{g}$, where $E$ is a supersingular elliptic curve. The set $\Phi_{g}$ of all elementary sequences of length $g$ has cardinality $\#\left(\Phi_{g}\right)=2^{g}$.

(1.1) Theorem. The unique one-dimensional stratum $S_{\{0, \cdots, 0,1\}}$ equals $L^{0}$, and its Zariski closure is

$$
L=\left(S_{\{0, \cdots, 0,1\}}\right)^{c},
$$

where $L^{0} \subset L$ are as constructed in (7.1). The closed curve $L \subset \mathcal{A}$ is connected.

(1.2) Theorem. Every stratum $S_{\varphi} \subset \mathcal{A}$ is non-empty and is quasi-affine (i.e. dense-open in an affine scheme). For every elementary sequence $\varphi$ all components of $S_{\varphi}$ have the same dimension and we have:

$$
\operatorname{dim}\left(S_{\varphi}\right)=|\varphi|:=\sum_{i=1}^{i=g} \varphi(i) .
$$




$$
\mathcal{A}=\bigsqcup_{\varphi} S_{\varphi}
$$

is a finite stratification: the boundary $\partial_{\mathcal{A}}\left(S_{\varphi}\right):=\left(S_{\varphi}\right)^{c}-S_{\varphi}$ is the union of all strata meeting that boundary,

$$
\partial_{\mathcal{A}}\left(S_{\varphi}\right)=\bigsqcup_{\varphi^{\prime}} S_{\varphi^{\prime}} \text {, the union taken over } \forall\left(S_{\varphi}\right)^{c} \cap S_{\varphi^{\prime}} \neq \emptyset ;
$$

i.e. either $S_{\varphi^{\prime}}$ does not meet $\left(S_{\varphi}\right)^{c}$, or it is contained in $\left(S_{\varphi}\right)^{c}$.

For every $\varphi \in \Phi$, with $\varphi$ not superspecial, and every irreducible component $S \subset S_{\varphi}$, there exists an irreducible component $L^{\prime} \subset L$ contained in $S^{c}$. Hence, every stratum $S_{\varphi}$ with $\varphi \neq\{0, \cdots, 0\}$, i.e. which is not the superspecial locus, has the property that $\left(S_{\varphi}\right)^{c}$ is connected.

(1.4) Corollary (Faltings, Chai). Let $k$ be an algebraically closed field, and let $n \in \mathbb{Z}_{>0}$, such that $\operatorname{char}(k)$ does not divide $n$. The moduli space $\mathcal{A}_{g, 1, n} \otimes k$ is irreducible.

(1.5) Corollary. Consider $V_{g-1} \subset \mathcal{A}_{g, 1, n} \otimes \mathbb{F}_{p, n}$, the locus of the nonordinary principally polarized abelian varieties. Suppose $g>1$. This locus is geometrically irreducible.

(1.6) "Polarized $\mathrm{BT}_{1}$ truncated group schemes". For a complete classification of all $(N,<,>)=(X, \lambda)[p]$, where $N=X[p]$ over an algebraically closed field $k$, see Section 9.

(1.7) For a complete classification of all principally polarized abelian varieties $(X, \lambda)$ in characteristic $p$ with $a(X)=g-1$, see Section 8 .

\section{Filtrations on finite group schemes}

In this section we define the canonical filtration on certain finite group schemes. We derive some elementary properties.

(2.1) All base schemes in this paper will be in characteristic $p$. All finite group schemes considered will be commutative. If we consider $N \rightarrow S$, a group scheme over a base, it is supposed to be finite and flat over $S$.

We write $F: N \rightarrow N^{(p)}$ for the Frobenius homomorphism and $V: N^{(p)} \rightarrow$ $N$ for the Verschiebung homomorphism. All finite group schemes considered in this section will be annihilated by $p$. 
For a group scheme $N \rightarrow S$, commutative and finite and flat over $S$, we will write $N[F]:=\operatorname{Ker}\left(F: N \rightarrow N^{(p)}\right)$, we write $V(N)=\operatorname{Im}\left(V: N^{(p)} \rightarrow N\right)$, if these exist as finite flat group schemes over $S$; if this is the case we say that $N / S$ is a Barsotti-Tate truncated level one group scheme if it is annihilated by $p$, i.e. $[p]_{N}=0$, and

$\operatorname{Im}\left(V: N^{(p)} \rightarrow N\right)=\operatorname{Ker}\left(F: N \rightarrow N^{(p)}\right), \operatorname{Im}\left(F: N \rightarrow N^{(p)}\right)=\operatorname{Ker}\left(V: N^{(p)} \rightarrow N\right)$.

Such a group scheme will be called a $\mathrm{BT}_{1}$.

Suppose $T \subset N$ a subgroup scheme. We will write $F^{-1}(T) \subset N$ for the finite flat subgroup scheme (if it exists as a finite flat group scheme over $S$ ) which is the pull back by $F: N \rightarrow N^{(p)}$ of $T^{(p)} \subset N^{(p)}$

(2.2) Construction: the canonical filtration. We suppose $N$ is a $\mathrm{BT}_{1}$ over a field $K \supset \mathbb{E}_{p}$. We construct a filtration:

$$
0=N_{0} \subset \cdots \subset N_{r}=V(N) \subset \cdots \subset N_{s}=N \text {; }
$$

here

$$
N_{r}=H:=V(N)=N[F],
$$

and $0 \leq r \leq s$ are integers. The construction is as follows: in the first step on considers all images $V^{i}(N)$; this gives a filtration of $N$; in the second step we take all $F^{-j}\left(N^{\prime}\right), \quad j \in \mathbb{Z}_{>0}$, for every $N^{\prime}$ in the previous filtration; then we go on by induction: in all odd numbered steps we take all $V^{i}\left(N^{\prime}\right)$, for all $N^{\prime}$ in the previous filtration; in all even numbered steps we take all $F^{-j}\left(N^{\prime}\right)$. Each step gives a filtration which is a refinement of the previous one. Note that odd numbered steps add at most new steps in the filtration of $0 \subset H$, and even numbered steps add at most new steps in the filtration of $H \subset N$. After a finite number of steps the process stabilizes, in fact after at most $2(q-1)$ steps if $\operatorname{rk}(N)=p^{q}$; the filtration reached will be called the canonical filtration of $N$.

Let $E$ be the set of all finite words in the symbols $V$ and $F^{-1}$. The set of subgroup schemes $\{W(N) \mid W \in E\}$ is the canonical filtration of $N$.

Note that we performed construction of the canonical filtration for a $\mathrm{BT}_{1}$ over a field. For a group scheme over an arbitrary base the canonical filtration need not exist (steps might produce non-flat group schemes). We will come back to this.

(2.3) Notation. A $\mathrm{BT}_{1}$ over a field $K$ has a canonical filtration, and we derive a triple $\tau(N)=\tau=\{v, f, \rho\}$, where:

$\rho:\{0, \cdots, s\} \rightarrow \mathbb{Z}_{\geq 0}, \quad v:\{0, \cdots, s\} \rightarrow\{0, \cdots, r\}, \quad f:\{0, \cdots, s\} \rightarrow\{r, \cdots, s\}$, are defined by:

$$
\operatorname{rk}\left(N_{i}\right)=p^{\rho(i)}, \quad V\left(N_{i}\right)=N_{v(i)}, \quad F^{-1}\left(N_{i}\right)=N_{f(i)} .
$$


The triple $\{v, f, \rho\}$ will be called the canonical type of $N$; it will be denoted by $\tau(N)$. We describe some properties. We write $\Gamma=\Gamma_{s}=\{0, \cdots, s-1\}$. We define $\pi_{\tau}=\pi: \Gamma \rightarrow \Gamma$ by:

$$
v(i+1)>v(i) \Rightarrow \pi(i):=v(i) ; \quad v(i+1)=v(i) \Rightarrow \pi(i):=f(i) .
$$

We write $B_{i}=N_{i+1} / N_{i}$ for every $i \in \Gamma$. Note that the maps $v$ and $f$ are monotone, i. e. $v(i+1) \geq v(i)$ and $f(i+1) \geq f(i)$ for all $i$.

(2.4) Lemma. Let $N$ be a $B T_{1}$ over a field $K$, and let $\{v, f, \rho\}$ be its canonical type. Then:

(i) the maps $v:\{0, \cdots, s\} \rightarrow\{0, \cdots, r\}$ and $f:\{0, \cdots, s\} \rightarrow\{r, \cdots, s\}$ are surjective; the map $\pi: \Gamma \rightarrow \Gamma$ is bijective.

(ii)

$$
v(i+1)>v(i) \Longleftrightarrow f(i+1)=f(i) ; \quad \text { in this case } \quad V: B_{i}^{(p)} \stackrel{\sim}{\longrightarrow} B_{\pi(i)}
$$

is an isomorphism;

(iii)

$$
v(i+1)=v(i) \Longleftrightarrow f(i+1)>f(i) ; \quad \text { in this case } \quad F: B_{\pi(i)} \stackrel{\sim}{\longrightarrow} B_{i}^{(p)}
$$

is an isomorphism;

(iv)

$$
f(i)+v(i)=s+i
$$

Remark. Consider the set $E$ of all finite words in the letter $V$ and $F^{-1}$. For $w \in E$ and a $\mathrm{BT}_{1}$ over a field as above we consider the rank of $w(N)$. The group scheme $N$ determines a function $E \rightarrow \mathbb{Z}$ by $w \mapsto \operatorname{rk}(w(N))$; we write this function as indexed set $\{\operatorname{rk}(w(N)) \mid w \in E\}$; if these functions are equal for $N_{1}$ and $N_{2}$, then $\tau\left(N_{1}\right)=\tau\left(N_{2}\right)$.

(2.5) Notation: Cycles. The bijection $\pi$ splits up $\Gamma$ into cycles, orbits under the group generated by $\pi$ :

$$
\Gamma=\Gamma_{1} \sqcup \cdots \sqcup \Gamma_{m} .
$$

For an element $i \in \Gamma$ we say $n \in \mathbb{Z}_{>0}$ is the order if $n$ is the smallest positive number such that $\pi^{n}(i)=i$, i.e. if $i \in \Gamma_{b}$ then $n=\#\left(\Gamma_{b}\right)$.

Proof of (2.4). In the canonical filtration every $0 \subset N_{i} \subset H=V(N)$ is the image under $V$ of a group scheme in the filtration (this is the way these 
$N_{i}$ are constructed; hence $v:\{0, \cdots, s\} \rightarrow\{0, \cdots, r\}$ is surjective. The same arguments for $H \subset N_{i} \subset N$ show that $f$ is surjective. We show that $\pi: \Gamma \rightarrow \Gamma$ is injective; indeed, suppose $\pi\left(i_{1}\right)=\pi\left(i_{2}\right)<r$; then $\pi\left(i_{1}\right)=v\left(i_{1}\right)=b=v\left(i_{2}\right)=$ $\pi\left(i_{2}\right)$; as $v$ is monotone, the fact $v\left(i_{1}+1\right)>v\left(i_{1}\right)=b=v\left(i_{2}\right)<v\left(i_{2}+1\right)$ implies $i_{1}=i_{2}$ (there is precisely one $i$ at which $v(i)$ jumps from $b$ to $b+1$ ); note that $\pi(i) \geq r$ implies $v(i+1)=v(i)$; if $\pi\left(i_{1}\right)=\pi\left(i_{2}\right) \geq r$ the same arguments involving $f$ show that $i_{1}=i_{2}$; this proves that $\pi: \Gamma \rightarrow \Gamma$ is bijective.

As $\pi$ is bijective, we obtain the cycle structure on $\Gamma$ as indicated above.

Claim. For every $i \in \Gamma$ we have $\operatorname{rk}\left(B_{i}\right) \geq \operatorname{rk}\left(B_{\pi(i)}\right)$. For every $i \in \Gamma$ the homomorphism $V: B_{i}^{(p)} \rightarrow N_{v(i+1)} / N_{v(i)}$ is surjective, and $F: N_{f(i+1)} / N_{f(i)} \rightarrow$ $\left(N_{i+1} / N_{i}\right)^{(p)}$ is injective. this proves the claim.

Following the elements in a cycle under $\pi$, we see that

$$
\operatorname{rk}\left(B_{i}\right) \geq \operatorname{rk}\left(B_{\pi(i)}\right) \geq \cdots \geq \operatorname{rk}\left(B_{\pi^{n}(i)}\right)=\operatorname{rk}\left(B_{i}\right) .
$$

We conclude that $v(i+1)>v(i)$ implies that

$$
V: B_{i}^{(p)} \stackrel{\sim}{\longrightarrow} N_{v(i+1)} / N_{v(i)}=B_{v(i)}
$$

is an isomorphism. This shows that $\left(\operatorname{Ker}(V) \cap N_{i+1}\right) \subset N_{i}$; hence we conclude that $v(i+1)>v(i) \Rightarrow f(i+1)=f(i)$.

In the same way we show that $f(i+1)>f(i)$ implies that

$$
F: N_{f(i+1)} / N_{f(i)}=B_{f(i)} \stackrel{\sim}{\longrightarrow} B_{i}^{(p)}
$$

is an isomorphism and that $v(i+1)=v(i)$.

Clearly $f(0)+v(0)=r+0$; by (ii) and (iii) the last claim follows. This concludes the proof of Lemma (2.4).

(2.6) Corollary. Let $i \in \Gamma$, and let $n \in \mathbb{Z}_{>0}$ be the order of $i$ under $\pi$. The maps in (ii) and the inverses of the maps in (ii) above give a (canonical) isomorphism

$$
B_{i}^{\left(p^{n}\right)} \stackrel{\sim}{\longrightarrow} B_{i}
$$

(2.7) Definition. A triple $\tau=\{v, f, \rho\}$ is a canonical type if $0 \leq r \leq s$ are integers, $v, f$ and $\rho$ are maps as in (2.3), such that:

- $\rho:\{0, \cdots, s\} \rightarrow \mathbb{Z}_{\geq 0}$ is strict monotone with $\rho(0)=0$,

- the maps $v$ and $f$ are monotone and surjective, with

$v(i+1)>v(i) \Longleftrightarrow f(i+1)=f(i), \quad v(i+1)=v(i) \Longleftrightarrow f(i+1)>f(i) ;$

this defines a permutation $\pi$ of $\Gamma:=\{0, \cdots, s-1\}$; 
- $\rho(i+1)-\rho(i)=\rho(\pi(i+1))-\rho(\pi(i))$ for every $i \in \Gamma$.

We have seen that a $\mathrm{BT}_{1}$ over a field defines a canonical type. Conversely:

(2.8) Remark. For every canonical type, there exists a $\mathrm{BT}_{1}$ over a field (even over $\mathbb{F}_{p}$ ) having this canonical type. We will not use this; this simple observation can be easily proved with methods indicated later.

Methods of this section can be easily derived from [27]. In Section 5 we will give more details on filtrations, and we give a classification of symmetric $\mathrm{BT}_{1}$ group schemes. All these methods will be easy, and in fact almost equivalent to [27]. Then, in Section 9 we will classify "polarized $\mathrm{BT}_{1}$ truncated group schemes"; this will be the backbone of the deformation theory in later sections.

(2.9) Remark. In the case of symmetric $\mathrm{BT}_{1}$ 's we will study the possible canonical types more in detail in Section 5 .

Cycles studied in this section in $\Gamma$ will be called $\left(V, F^{-1}\right)$-cycles, in contrast with " $(V, \perp)$-cycles" studied later.

\section{Strata: the canonical stratification of the moduli space of abelian varieties}

(3.1) Lemma. Let $h: M_{1} \rightarrow M_{2}$ be a homomorphism of finite flat group schemes over a noetherian scheme $S$; let $R$ be a positive integer. Consider all points $s \in S$ such that $\operatorname{Im}\left(h_{s}: M_{1, s} \rightarrow M_{2, s}\right)$ has rank equal to $R$. These points give a locally closed set $S(R) \subset S$; this is a locally closed, reduced subscheme, and $\left.\left.\operatorname{Im}\left(h: M_{1} \rightarrow M_{2}\right)\right|_{S(R)} \subset M_{2}\right|_{S(R)}$ exists as a finite flat group scheme.

Let $h: M_{1} \rightarrow M_{2}$ be a flat surjective homomorphism of finite flat group schemes over a noetherian scheme $S$, and let $N_{2} \subset M_{2}$ be a flat subgroup scheme; let $R$ be a positive integer. Consider all points $s \in S$ such that $h^{-1}\left(\left(N_{2}\right)_{s}\right)$ has rank equal to $R$. These points give a locally closed set $T(R) \subset S$; this is a locally closed, reduced subscheme, and $\left.\left.h^{-1}\left(N_{2}\right)\right|_{T(R)} \subset M_{1}\right|_{T(R)}$ exists as a finite flat group scheme.

Proof. The rank of the fibers of the finite group scheme $\operatorname{Ker}\left(h: M_{1} \rightarrow M_{2}\right)$ is upper-semicontinuous. Hence $S(R) \subset S$ is locally closed. Over this scheme $\left.\operatorname{Ker}(h)\right|_{S(R)}$ is finite with geometric fibers of constant rank; hence it is flat by [4], Lemma (1.13) on page 240. Hence the quotient $\left.M_{1}\right|_{S(R)} /\left.\operatorname{Ker}(h)\right|_{S(R)}$ exists as finite, flat group scheme, see [14], page 212-17, Th. 7.2; this is a closed subscheme of $\left.M_{2}\right|_{S(R)}$, the image of $\left.h\right|_{S(R)}$. The same arguments show the second part of the claim.

Notation. Let $\tau$ be a canonical type, and let $N \rightarrow S$ be a BT $_{1}$ over $S$. We 
denote by $\mathcal{D}_{\tau}(S)$ the set, considered as reduced subscheme, of points $s \in S$ such that the fiber $N_{s}$ has canonical type $\tau\left(N_{s}\right)=\tau$.

(3.2) Proposition. Let $\tau$ be given, and let $N \rightarrow S$ be a $\mathrm{BT}_{1}$. Then

$$
\mathcal{D}_{\tau}(S) \subset S
$$

is locally closed.

Let a canonical type $\tau$ be given, and let $N \rightarrow T$ be a $\mathrm{BT}_{1}$, with $T$ reduced, such that for every $s \in T$ we have $\tau\left(N_{s}\right)=\tau$, i.e. "the type in the fibers of $N \rightarrow T$ is constant". Then the canonical filtration of $N / T$ exists, i.e. there exist finite flat subgroup schemes $N_{i} \subset N \rightarrow S$, which are obtained by the construction of the canonical filtration, and which in every fiber $N_{s}$ give the canonical flltration.

Proof. As we have seen, $\tau\left(N_{s}\right)$ is determined by the set $\{\operatorname{rk}(N) \mid w \in \mathcal{W}\}$. It suffices to consider only words of length at most $2 q(q-1)$, where $q=\operatorname{rk}(N)$. Consider the collection of all such words; for a given type $\tau$ fix the ranks of the images of each word belonging to the given type $\tau$. Start with $S=S_{0}$. Suppose $b \in \mathbb{Z}_{>0}$, suppose we work over a base $S_{b-1}$, and suppose that all words of length at most $b-1$ have "images" which are flat group schemes over $S_{b-1}$ of the given ranks. The set $S_{b} \subset S_{b-1}$ is the reduced locally closed subscheme where the image of every word of length $b$ has a given rank (determined by $\tau)$. This exists: use the previous lemma. Repeating this argument for all words and all ranks we have obtained from $\tau$ we obtain a locally closed set $S_{2 q(q-1)}$ in the base where all "images" have constant ranks, and give flat group schemes. This is the set we are looking for.

If $\tau(-)$ is constant on $T$, in each step of the proof of the previous lemma we obtain subgroup scheme of constant rank in $N$, hence flat subgroup schemes by [4], Lemma (1.13) on page 240. This shows that the construction of the canonical filtration under the condition $\tau(-)$ is constant on $T$ produces a filtration by flat subgroup schemes, and the proposition is proved.

Obviously the canonical filtration on a group scheme over an arbitrary base does not exist if the type changes.

(3.3) Notation. Let $\tau$ be a canonical type. For a fixed $n \in \mathbb{Z}_{>0}$ consider the moduli space

$$
\mathcal{A}^{\prime}:=\mathcal{A}_{g, *, n} \otimes \mathbb{F}_{p, n}=\cup_{d} \quad \mathcal{A}_{g, d, n} \otimes \mathbb{F}_{p, n}
$$

We write

$$
D_{\tau}:=\mathcal{D}_{\tau}\left(\mathcal{A}^{\prime}\right) \subset \mathcal{A}^{\prime}
$$

for the locally closed reduced subscheme consisting of moduli points $s \in \mathcal{A}^{\prime}$, where $s$ comes over some field from an abelian variety $X_{s}$, such that $\tau\left(X_{s}[p]\right)=$ $\tau$. This is a locally closed closed subset. Note that the rank of $X_{s}[p]$ equals $2 g$. Note that only a finite number of canonical types exist for a given rank. 
(3.4) Definition. We have obtained a finite disjoint union into locally closed subsets:

$$
\mathcal{A}^{\prime}=\bigsqcup_{\tau} D_{\tau} .
$$

This is called the canonical stratification of $\mathcal{A}^{\prime}$. In some papers this is called the EO-stratification, or the Oort stratification.

(3.5) Remark. We study some properties of this stratification on $\mathcal{A}^{\prime}$. Then, in order to describe more precise results we will restrict later to the case of

$$
\mathcal{A}=\mathcal{A}_{g, 1, n} \otimes \mathbb{F}_{p, n},
$$

the moduli space of principally polarized abelian varieties in characteristic $p$.

\section{The Raynaud trick on $\mathcal{A}_{g} \otimes \mathbb{F}_{p}$}

(4.1) Theorem. For every canonical type $\tau$ every irreducible component of the stratum $D_{\tau} \subset \mathcal{A}^{\prime}:=\cup_{d} \mathcal{A}_{g, d, n} \otimes \mathbb{F}_{p, n}$ is a quasi-affine subscheme.

Proof. It suffices to show this in case of arbitrary high level structure. Hence we suppose $\mathcal{A}^{\prime}=\cup_{d} \mathcal{A}_{g, d, n} \otimes \mathbb{F}_{p, n}$, with $n \in \mathbb{Z}_{\geq 3}$ not divisible by $p$. We choose a component $T \subset D_{\tau} \subset \mathcal{A}^{\prime}$.

We are going to show that the theorem follows from ampleness of the determinant line bundle associated with the sheaf of invariant differential forms on the "universal semi-abelian scheme", as proved by Moret-Bailly, see [35], also see [10], combined with the properties we proved on cycles in the $p$-kernel constructed by the canonical filtration, see (2.4).

Let $\mathcal{X} \rightarrow \mathcal{A}^{\prime}$ be the universal abelian scheme. We write $\mathfrak{t}_{\mathcal{X} / \mathcal{A}}$ for the tangential sheaf of $\mathcal{X} \rightarrow \mathcal{A}^{\prime}$ along the zero-section, and we write $\omega_{\mathcal{X}} / \mathcal{A}^{\prime}$ for its determinant line bundle of its dual:

$$
\omega_{\mathcal{X} / \mathcal{A}^{\prime}}=\operatorname{Det}\left(\mathfrak{t}_{\mathcal{X}^{\prime} / \mathcal{A}^{\prime}}^{\text {unal }}\right)=\operatorname{Det}\left(\Omega_{\mathcal{X}^{\prime} / \mathcal{A}^{\prime}}\right),
$$

see [10], page 24 and page 137.

Claims. 1) Under these conditions, the line bundle $\omega_{\mathcal{X} / T}$ on $T$ is a torsion line bundle.

2) From this it follows that $T$ is quasi-affine.

By [35], Chap. IX, and [10], I.5 and V.2, Th. 2.3, we conclude that $\left.\omega\right|_{T}$ is ample; if we assume (1) it is also torsion; hence $T$ is quasi-affine. This proves: (1) $\Rightarrow$ (2).

We show that (1) is true. We write $N:=\left.\mathcal{X}[p]\right|_{T}$. By (3.2) we see that the canonical filtration $\left\{N_{i} \mid 0 \leq i \leq s\right\}$ by finite flat subgroup schemes of $N$ 
exists. We have:

$$
0=N_{0} \subset N_{1} \subset \cdots \subset N_{r}=N[F]=V(N)
$$

we see that all tangential sheaves

$$
\mathfrak{t}_{N_{1} / T} \subset \cdots \subset \mathfrak{t}_{N_{r} / T}=\mathfrak{t}_{\mathcal{X}^{\prime} / T}
$$

are well-defined, of constant rank. We write $\mathcal{L}_{i}:=\operatorname{Det}\left(\mathfrak{t}_{N_{i+1} / T} / \mathfrak{t}_{N_{i} / T}\right)$, for $0 \leq i<r$, and we are going to show that these are torsion line bundles. By (2.4) we know that if $i \in \Gamma$ has order $n(i)=n>0$ under $\pi: \Gamma \rightarrow \Gamma$, then for $B_{i}:=N_{i+1} / N_{i}$ we have

$$
\left(B_{i}\right)^{\left(p^{n}\right)} \cong B_{i} ; \text { hence }\left(\mathcal{L}_{i}\right)^{p^{n}} \cong \mathcal{L}_{i} ;
$$

this shows that $\operatorname{Det}\left(\mathcal{L}_{i}\right)^{p^{n}-1}=1$. We conclude that $\operatorname{Det}\left(\mathfrak{t}_{\mathcal{X}} / T\right)=\operatorname{Det}\left(\mathcal{L}_{1}\right) \otimes$ $\cdots \otimes \operatorname{Det}\left(\mathcal{L}_{r}\right)$ is torsion; hence its inverse $\left.\omega\right|_{T}$ is torsion. This shows (1), and it finishes the proof of Theorem (4.1)

(4.2) Remark. A special case of this proposition is due to Raynaud: if $S$ is irreducible and complete, in characteristic $p$, and $\mathcal{X} \rightarrow S$ is a family of ordinary abelian varieties (i.e. all fibers have maximal $p$-rank), then the natural map $S \rightarrow \mathcal{A}_{g} \otimes \mathbb{F}_{p}$ is constant, i.e. the family $\mathcal{X} \rightarrow S$ is isotrivial, cf. [60], Th. 5, page 62 , cf. [35], XI.5, page 237. This beautiful idea by Raynaud stimulated us to look for some of the basic notions as exposed in this paper.

The example of $p$-rank equal to $f=g-1$ is worked out in [45], 6.2; that case can be seen as a motivating example illustrating the proof of the "Raynaud trick" in the general setting, as given above.

In the case of a family of ordinary abelian varieties the tangent bundle can be trivialized by a finite covering, because " $\mu_{p}$ is rigid"; that was the original idea by Raynaud. In case of lower $p$-rank, the fact that $\alpha_{p}$ does not admit a canonical base seems to spoil the case; however then we consider the $p$-kernel $\mathcal{X}[p]$, "go up and down" by $V$ and by $F^{-1}$ we prove that the determinant of the tangent bundle is torsion, as is done above.

From now on abelian varieties considered will be principally polarized.

\section{Filtrations on finite symmetric group schemes}

In this section we consider symmetric $\mathrm{BT}_{1}$ 's, and we describe and characterize filtrations on such finite group schemes.

Finite group schemes will be considered, usually over a field, sometimes (finite and flat) over a more general base. We will write $N$ for a finite (commutative) group scheme. All finite group schemes considered in this section will 
be annihilated by $p$; when over an arbitrary base we suppose the group scheme is finite and flat over that base. In all cases we assume that we work with a $\mathrm{BT}_{1}$.

(5.1) Definition. We say that $\zeta$ is a symmetry on a finite group scheme $N$ if

$$
\zeta: N \stackrel{\sim}{\longrightarrow} N^{D}
$$

is an isomorphism onto its Cartier dual group scheme. A finite group scheme which admits a symmetry is called a symmetric finite group scheme.

Remark. Later we will be more precise whether this symmetry has extra properties (like being alternating or anti-symmetric).

For commutative group schemes we have

$$
\left(F_{N}: N \rightarrow N^{(p)}\right)^{D}=\left(V_{N^{D}}: N^{(p) D}=N^{D(p)} \rightarrow N^{D}\right)
$$

as $V$ commutes with base change we derive

$$
\zeta \cdot V_{N}=V_{N^{D}} \cdot \zeta^{(p)}=\left(F_{N}\right)^{D} \cdot \zeta^{(p)} .
$$

Notation. Suppose $\zeta: N \rightarrow N^{D}$ is a symmetry, and $i: T \hookrightarrow N$ is a finite subgroup scheme; we write:

$$
\perp_{\zeta}(T)=\perp(T):=\operatorname{Ker}\left(N \stackrel{\zeta}{\longrightarrow} N^{D} \stackrel{i^{D}}{\longrightarrow} T^{D}\right) .
$$

Earlier we have considered the set $E$ of all (finite) words in the symbols $V$ and $F^{-1}$. For a $\mathrm{BT}_{1}$ group scheme $N$ over a field the filtration $\{W(N) \mid W \in E\}$ was called the canonical filtration.

Consider the set $e$ of all (finite) words in the symbols $V$ and $\perp$. We consider the set of finite subgroup schemes $\{w(N) \mid w \in e\}$ of $N$.

(5.2) Lemma. Let $N$ be a finite group scheme over a field $K$. Assume this is a $\mathrm{BT}_{1}$. Assume there is a symmetry $\zeta: N \stackrel{\sim}{\longrightarrow} N^{D}$ on $N$. Then the following properties hold:

1) $\perp(\perp(T))=T$;

2) $\perp(V(N))=V(N)$;

3) the set $\{w(N) \mid w \in e\}$ is a finite filtration on $N$;

4) $\perp(V(T))=F^{-1}(\perp(T))$ for every subgroup scheme $T \subset N$;

5) $\{w(N) \mid w \in e\}=\{W(N) \mid W \in E\}$.

Remark. The last property shows that the canonical filtration does not depend 
on a particular choice of the symmetry.

Proof. 1) The first property follows by dualizing

$$
\perp(T) \rightarrow N \stackrel{\zeta}{\longrightarrow} N^{D} \rightarrow T^{D} .
$$

2) The second follows from: $\perp(V(N))=\operatorname{Ker}(F)=V(N)$.

3) Note that $\perp(N)=0$; hence for every word of the form $w=w^{\prime} \cdot\left(\perp \cdot V^{i} \cdot \perp\right)$ we have $w(N)=w^{\prime}(N)$. Hence we need only consider words ending with a positive power of $V$. The set $\left\{V^{i}(N) \mid i \in \mathbb{Z}_{>0}\right\}$ is a filtration of $V(N)$ which stabilizes after a finite number of steps. The set $\left\{\perp\left(V^{i}(N)\right) \mid i \in \mathbb{Z}_{>0}\right\}$ is a filtration of $V(N) \subset N$. Hence $\left\{\left(V^{j} \cdot \perp \cdot V^{i}\right)(N) \mid i, j \in \mathbb{Z}_{\geq 0}\right\}$ is a filtration, etc.: we carry on by induction on the number of letters in the words in consideration; this shows that we obtain a filtration; because $N$ is a finite group scheme, this is a finite filtration.

4) This follows because

$$
\left(F: N \rightarrow N^{(p)}\right)^{D}=\left(V:\left(N^{(p)}\right)^{D}=\left(N^{D}\right)^{(p)} \rightarrow N^{D}\right) .
$$

5 ) As we have seen, we need only consider words $w$ ending with $V$, and the same for words $W$. If $w=V \in e$, then also $w \in E$. If $w=w^{\prime} \cdot \perp \cdot V$, then $w(N)=\left(w^{\prime} \cdot V\right)(N)$. Suppose $w$ ends in $V^{2}$; note that if $i, j \in \mathbb{Z}_{\geq 0}$ then

$$
\left(V^{i} \cdot \perp \cdot V^{j} \cdot \perp\right)(T)=\left(V^{i} \cdot F^{-j}\right)(T) .
$$

We write either $w=w^{\prime} \cdot V^{b}$ (in case $\perp$ appears an even number of times in $w$ ), or $w=w^{\prime} \cdot V^{a} \cdot \perp \cdot V^{b}$ (in the odd case), where $a \in \mathbb{Z}_{>0}$, and $b \in \mathbb{Z}_{>1}$, and where $w^{\prime}$ is a finite product of factors of the form $V^{i} \cdot \perp \cdot V^{j} \cdot \perp$; in the second case, note that

$$
w(N)=\left(w^{\prime} \cdot V^{a} \cdot \perp \cdot V^{b}\right)(N)=\left(w^{\prime} \cdot V^{a} \cdot F^{b-1} \cdot V\right)(N) ;
$$

hence in both cases there exists a word $W \in E$ such that $w(N)=W(N)$. Hence

$$
\{w(N) \mid w \in e\} \subset\{W(N) \mid W \in E\} .
$$

Conversely, considering all $V^{i} \cdot F^{-j}$, with $i \geq 0$, in a word $W$ ending on $V$ shows the opposite inclusion. This ends the proof of the lemma.

(5.3) Definition. Let $(N, \zeta)$ be a symmetric $\mathrm{BT}_{1}$ over a base $S$. We say that

$$
0=N_{0} \subset \cdots \subset N_{i} \subset \cdots \subset N_{r}=H=V N=N[F] \subset \cdots \subset N_{2 r}=N
$$

is a good filtration if the following conditions are satisfied:

1) Each $N_{j} \rightarrow S$ is a flat subgroup scheme, $N_{i} \neq N_{i+1}$.

2) For every $0 \leq j \leq 2 r$ duality gives an equality

$$
\perp\left(N_{j}\right)=N_{2 r-j} .
$$


3) For every $0 \leq j \leq 2 r$, the image $\operatorname{Im}\left(V: N_{j}^{(p)} \rightarrow N\right)$ exists as a finite flat group scheme over $S$, and it equals one of the group schemes in the filtration, and

4) for every $0 \leq i \leq r$, the group scheme $N_{i}$ is such an image.

We say that a good filtration is a final fltration if moreover its length equals $2 g$ with $p^{2 g}:=\operatorname{rk}(N / S)$ :

$$
0=N_{0} \subset \cdots \subset N_{g}=V(N) \subset \cdots \subset N_{2 g}=N
$$

by finite, flat group schemes over $S$, such that:

5) the rank of $N_{i}$ over $S$ equals $p^{i}$.

(5.4) Proposition. Let $N$ be a symmetric $\mathrm{BT}_{1}$ over a field. Its canonical flltration, as constructed in Section 2, is a good filtration.

Proof. In Lemma (5.2) we have seen that the canonical filtration constructed with the help of $V$ and $F^{-1}$ is the same as the one constructed with $V$ and $\perp$. Hence the canonical filtration is stable under $\perp$; hence it satisfies (2). Conditions (3) and (4) follow by construction.

We see that the canonical filtration (if it exists) is the "minimal good filtration" and a final filtration (if it exists) a "maximal good filtration".

(5.5) Proposition. Suppose that $N$ is a symmetric $\mathrm{BT}_{1}$ over a base $S \rightarrow \mathbb{E}_{p}$ with a good filtration

$$
0=N_{0} \subset \cdots \subset N_{i} \subset \cdots \subset N_{r}=H \subset \cdots \subset N_{2 r}=N
$$

on it. For every $j$ with $0 \leq j \leq 2 r$ we write $\nu(j)$ for the integer with the property

$$
\operatorname{Im}\left(V: N_{j}^{(p)} \rightarrow N\right)=N_{\nu(j)}
$$

1) For every $0 \leq j \leq 2 r$ the inverse image of $N_{j}^{(p)}$ under $F: N \rightarrow N^{(p)}$ equals

$$
F^{-1}\left(N_{j}^{(p)}\right)=N_{2 r-\nu(2 r-j)}
$$

2) For every $0 \leq j<2 r$ there is an exact sequence

$0 \rightarrow N_{2 r-\nu(2 r-j-1)} / N_{2 r-\nu(2 r-j)} \stackrel{F}{\rightarrow} N_{j+1}^{(p)} / N_{j}^{(p)} \stackrel{V}{\longrightarrow} N_{\nu(j+1)} / N_{\nu(j)} \rightarrow 0$.

3) For every $0 \leq j<2 r$

$$
\nu(j)=\nu(j+1) \quad \Longleftrightarrow \quad \nu(2 r-j)=\nu(2 r-j-1)+1 .
$$


4) If $0 \leq j<2 r$ and $\nu(j)<\nu(j+1)$, then $\nu(j)+1=\nu(j+1)$, and

$$
V: N_{j+1}^{(p)} / G_{j}^{(p)} \stackrel{\sim}{\longrightarrow} N_{\nu(j+1)} / N_{\nu(j)}
$$

Proof. From

$$
V\left(N_{2 r-j}^{(p)}\right) \subset N_{\nu(2 r-j)}
$$

we deduce

$$
F\left(N_{2 r-\nu(2 r-j)}\right) \subset N_{j}^{(p)} .
$$

Hence all arrows as claimed in the proposition exist. In order to show the statements it suffices to show these for every fiber $N_{s} \rightarrow \operatorname{Spec}(K) \subset S$, where $K$ is a perfect field. We write $A=\mathbb{D}\left(N_{s}\right)$ for its Dieudonné module.

1) In order to show $\mathcal{V}^{-1}\left(A_{j}^{(p)}\right)=A_{2 r-\nu(2 r-j)}$ we observe:

$$
\begin{gathered}
x \in \mathcal{V}^{-1}\left(A_{j}^{(p)}\right) \Longleftrightarrow \mathcal{V}(x) \in A_{j}^{(p)} \Longleftrightarrow \\
\Longleftrightarrow \forall y \in A_{2 r-j}, \quad<\mathcal{V} x, y>=0 \Longleftrightarrow \forall y \in A_{2 r-j}, \quad<x, \mathcal{F} y>=0 \Longleftrightarrow \\
\Longleftrightarrow \forall z \in \mathcal{F}\left(A_{2 r-j}^{(p)}\right)=A_{\nu(2 r-j)}, \quad<x, z>=0 \Longleftrightarrow x \in \perp\left(A_{\nu(2 r-j)}\right)=A_{2 r-\nu(2 r-j)} .
\end{gathered}
$$

2) The sequence as in (2) exists, surjectivity on the right follows from the definition of $\nu$ in a good filtration, and injectivity on the left follows from (1). In order to show exactness in the middle, we choose $x \in A_{j+1}^{(p)}$ such that

$$
\mathcal{F} x \in A_{\nu(j)}=\mathcal{F}\left(N_{j}^{(p)}\right)
$$

Let $y \in A_{j}^{(p)}$ with $\mathcal{F} x=\mathcal{F} y$. Then $\mathcal{F}(x-y)=0$, hence there exists $z$ with $\mathcal{V} z=x-y \in A_{j+1}^{(p)}$. By (1) this implies $z \in A_{2 r-\nu(2 r-j-1)}$. As $\mathcal{V} z$ and $x$ are congruent $\bmod A_{j}$ this proves exactness in the middle.

3) For any index $j<2 r$ we have $\nu(j+1) \leq \nu(j)+1$. If $\nu(j)=\nu(j+1)$ then by (2) we conclude $\nu(2 r-j)=\nu(2 r-j-1)+1$. Note that $\nu(2 r)=r$. Hence by the previous argument, it is excluded that for any $j$ we have $\nu(j)<\nu(j+1)$ ánd $\nu(2 r-j)<\nu(2 r-j-1)$. This proves $(3)$.

4) This follows from (2) and (3).

(5.6) Definition: Final types and elementary sequences. A final sequence is a map $\psi:\{1, \cdots, 2 g\} \rightarrow \mathbb{Z}_{\geq 0}$ with $\psi(0)=0$, and $\psi(2 g)=g$, such that

$$
\psi(i) \leq \psi(i+1) \leq \psi(i)+1, \quad 0 \leq i<2 g,
$$

and

$$
\psi(i)+1=\psi(i+1) \Longleftrightarrow \psi(2 g-1)=\psi(2 g-i-1)
$$


Note that this implies:

$$
\psi(2 g-i)=\psi(i)+g-i .
$$

An elementary sequence is a map $\varphi:\{1, \cdots, g\} \rightarrow \mathbb{Z}_{\geq 0}$ with $\varphi(0)=0$ and

$$
\varphi(i) \leq \varphi(i+1) \leq \varphi(i)+1, \quad 0 \leq i<g .
$$

A symmetric canonical type is a canonical type as in (2.3) with $s=2 r$ and the extra conditions

$$
f(j)=2 r-v(2 r-j), \quad \forall 0 \leq j<r
$$

and

$$
\rho(j+1)-\rho(j)=\rho(2 r-j)-\rho(2 r-j-1), \quad \forall 0 \leq j<r .
$$

Note that in a symmetric canonical type we have $f(j)=2 r-v(2 r-j)=$ $r+j-v(j)$ hence $v(2 r-j)-v(j)=r-j$ for all $j$. If $N$ is a symmetric $\mathrm{BT}_{1}$ over a field, its canonical filtration, given by $V$ and $F^{-1}$, defines a canonical type, and this is symmetric.

We could define a good type as a pair of maps $\nu:\{0, \cdots, 2 r\} \rightarrow\{0, \cdots, r\}$, and $\rho:\{0, \cdots, 2 r-1\} \rightarrow \mathbb{Z}_{>0}$ satisfying obvious rules generalizing the definition of a symmetric canonical type. Here $f$ is given by $f(j)=2 r-\nu(2 r-j)$. We will not need this definition.

The set of all final sequences for given value of $g$ is denoted by $\Psi=\Psi_{g}$. The set of all elementary sequences for given value of $g$ is denoted by $\Phi=\Phi_{g}$. Clearly $\#\left(\Phi_{g}\right)=2^{g}$ (in each step the sequence $\varphi$ either jumps by one, or does not jump).

We define a partial ordering on $\Phi$ by:

$$
\varphi^{\prime} \prec \varphi \stackrel{\text { def }}{\Longleftrightarrow} \varphi(i)^{\prime} \leq \varphi(i) \quad \forall i \leq g .
$$

Observe that any maximally totally ordered subset in $\Phi$ has length equal to $g(g+1) / 2$.

We define a "dimension function" $|\cdot|: \Phi \rightarrow \mathbb{Z}$ on $\Phi$, by:

$$
|\varphi|:=\sum_{i=1}^{i=g} \varphi(i)
$$

Note that for a final sequence we have $\psi(2 g-i)-\psi(i)=g-i$ for $0 \leq i<g$; we call this "symmetry". A final sequence $\psi$ defines an elementary sequence $\varphi$ by $\varphi(i):=\psi(i), \quad \forall 0 \leq i \leq g$; we call this "truncation" of the final sequence. Conversely, an elementary sequence $\varphi$ defines a final sequence $\psi$ by: $\psi(i)=$ $\varphi(i)=\psi(2 g-i)-g+i$ for $0 \leq i \leq g$; this is inverse to the "truncation" process. 
Combinatorial constructions. Suppose given a symmetric canonical type $\tau=\{v, f, \rho\}$. We associate to this a final sequence and an elementary sequence $\varphi=\operatorname{stretch}(\tau)$ by "stretching": suppose $\{\varphi(0), \varphi(1), \cdots, \varphi(\rho(i))\}$ is defined, for $0 \leq i$; then define $\{\varphi(0), \varphi(1), \cdots, \varphi(\rho(i+1))\}$ by $\varphi(\rho(i))=\varphi(\rho(i)+1)=$ $\cdots=\varphi(\rho(i+1))$ if $v(i+1)=v(i) ; \quad\{\varphi(0), \varphi(1), \cdots, \varphi(\rho(i+1))\}$ by $\varphi(\rho(i))<$ $\varphi(\rho(i)+1)<\cdots<\varphi(\rho(i+1))$ if $v(i+1)>v(i)$; clearly this procedure produces an elementary sequence.

Suppose given a final type. Single out those indices which can be reached by starting with $2 g$ and applying $j \rightarrow \psi(j)$ and $i \rightarrow 2 g-i$ as many times until no further indices appear (this we call the canonical construction); then delete all numbers not appearing, and construct a canonical type $\tau$ from these data in the obvious way.

Remark. We shall see that for a "polarized $\mathrm{BT}_{1}$ " over an algebraically closed field the canonical filtration can be refined to a final filtration, see (9.6). The final type associated to a canonical type by stretching is the the type of any final filtration associated with the canonical filtration. A refinement from a canonical filtration to a final filtration is not unique in general. However the final type and the elementary type are uniquely determined by the canonical type, and are the ones obtained by stretching and truncation.

(5.7) Lemma. Suppose given $g \in \mathbb{Z}_{>0}$. There is a natural bijection of sets:

(CT) all symmetric canonical types $\tau=\{v, f, \rho\}$ with $\rho(2 r)=2 g$,

(FT) all final sequences $\psi \in \Psi_{g}$,

(ES) all elementary sequences $\varphi \in \Phi_{g}$,

where $(\mathrm{CT}) \rightarrow \Phi$ is given by stretching, and $\Psi \rightarrow \Phi$ is given by truncation.

Proof. It is clear that stretching indeed produces an element in $\Phi$. From an elementary sequence $\varphi$ we define a final sequence by "symmetry"; this map $\Phi \rightarrow \Psi$ is left an right inverse to truncation. Given a final sequence (or a final filtration) we produce a canonical type (or a canonical filtration) by applying the construction of the canonical type (or construction of the canonical filtration). It is straightforward that all maps involved are bijective (and in fact inverse to each other where applicable).

(5.8) Notation. Let $N$ be a symmetric $\mathrm{BT}_{1}$ over a field; its canonical type defines an elementary sequence; this will be denoted by $\operatorname{ES}(N) \in \Phi$. For an abelian variety $X$ over a field, which admits a principal polarization; the isomorphism $X \stackrel{\sim}{\longrightarrow} X^{t}$, using the duality theorem, see [42], 19.1, shows that $X[p] \cong X^{t}[p] \cong X[p]^{D}$, and it follows that $N=X[p]$ is symmetric; we write $\operatorname{ES}(X):=\operatorname{ES}(X[p]) \in \Phi$.

(5.9) Cycles. Suppose given a canonical type (or a canonical filtration on a $\mathrm{BT}_{1}$ over a base). We have defined a $\left(V, F^{-1}\right)$-cycle in Section filt for a canonical 
type by

$$
\Gamma=\{0, \cdots, 2 r-1\}, \quad \pi: \Gamma \rightarrow \Gamma
$$

and we obtain a disjoint union

$$
\Gamma=\Gamma_{1} \sqcup \cdots \sqcup \Gamma_{m}
$$

of $\left(V, F^{-1}\right)$-cycles.

Suppose we have a symmetric canonical type. We generate an equivalence relation, the $(V, \perp)$-relation, by iterating:

$$
\nu(j)>\nu(j-1) \quad \Longrightarrow \quad j-1 \sim \nu(j-1)
$$

and

$$
j-1 \sim 2 r-j+1 .
$$

Explanation: in a canonical filtration, we declare the steps, where

$$
V: N_{j}^{(p)} / N_{j-1}^{(p)} \rightarrow N_{\nu(j)} / N_{\nu(j-1)}
$$

is an isomorphism, to be equivalent steps, and we declare dual steps under $\perp$ to be equivalent.

Remark. We can achieve the $\left(V, F^{-1}\right)$-equivalence and the $(V, \perp)$-equivalence as follows. Connect vertices in $\Gamma$ by ordered edges $V$ and $F^{(-1)}$ and by unordered edges $\perp$. Deleting the $\perp$-edges gives the $\left(V, F^{-1}\right)$-equivalence and the $\left(V, F^{-1}\right)$-cycles. Deleting the $F^{(-1)}$-edges gives the $(V, \perp)$-equivalence and the $(V, \perp)$-cycles. We easily see that a $(V, \perp)$-equivalence class consists of a graph which contains exactly one cycle and in each vertex $j$ in the cycle:

1) Either in the cycle an edge $\perp$ ends in $j$, and an edge $V$ starts; no $V$ ends in $j$.

2) Or an edge $V$ ends, an edge $V$ starts in $j$, and there is an edge $\perp$ attached to $j$, not included in the cycle;

3) Or an edge $V$ ends in $j$, an edge $\perp$ is attached to $j$, no $V$ starts in $j$; the cycle passing through $j$ reads:

$$
\cdots \stackrel{V}{\longrightarrow} j \stackrel{\perp}{\longleftrightarrow} 2 r-j+1 \stackrel{V}{\longrightarrow} \cdots ;
$$

only in the second case there is is an edge attached to $j$ not contained in the cycle ("a loose edge").

We study the relation between the $\left(V, F^{-1}\right)$-equivalence relation and the $(V, \perp)$-equivalence relation. 
(5.10) Lemma and definition. Suppose given a symmetric canonical type $\tau$. Let $\Gamma=\Gamma_{1} \sqcup \cdots \sqcup \Gamma_{m}$ be the disjoint union of $\left(V, F^{-1}\right)$-cycles. Then:

(i) The operation $\perp: \Gamma \rightarrow \Gamma$ respects the disjoint union into $\left(V, F^{-1}\right)$ cycles.

Suppose $\Gamma^{\prime}=\Gamma_{i}$ for some index $i$; then

(ii) odd either $\perp\left(\Gamma^{\prime}\right)=\Gamma^{\prime}$; in this case \#( $\left.\Gamma^{\prime}\right)$ is an even number; in this case there is precisely one $(V, \perp)$-cycle in $\Gamma^{\prime}$ and $\Gamma^{\prime}$ is a $(V, \perp)$-equivalence class; this $(V, \perp)$-cycle in $\Gamma^{\prime}$ contains an odd number of symbols $\perp$; this will be called an odd cycle; in this case the length of the $(V, \perp)$-cycle is this odd number plus $\#\left(\Gamma^{\prime}\right) / 2$,

(iii) even or $\perp\left(\Gamma^{\prime}\right)=\Gamma^{\prime \prime} \neq \Gamma^{\prime}$; in this case $\#\left(\Gamma^{\prime} \cup \Gamma^{\prime \prime}\right)$ is a $(V, \perp)$ equivalence class; in this case the $(V, \perp)$-cycle in $\Gamma^{\prime} \cup \Gamma^{\prime \prime}$ contains an even number of symbols $\perp$; this will be called an even cycle.

Proof. By construction we have $\pi \cdot \perp=\perp \cdot \pi$. This proves (i).

Suppose $\Gamma^{\prime}=\Gamma_{i}=\perp\left(\Gamma^{\prime}\right)$ for some index $i$. Let $j \in \Gamma^{\prime}$. Then $\perp(j) \in$ $\perp\left(\Gamma^{\prime}\right)=\Gamma^{\prime}$. Let $w$ be the shortest word in $V$ and $F^{-1}$ with $w(j)=\perp(j)$. Then $\bar{w}(\perp(j))=j$, where $\bar{w}$ is the word obtained from $w$ by replacing $V$ by $F^{-1}$ and $F^{-1}$ by $V$. Hence $\#\left(\Gamma^{\prime}\right)$ is even. In the circular graph $\Gamma^{\prime}$ every vertex is connected to its next either by $V$ or by $F^{-1}$, and for opposite vertices one is $V$, and the opposite is $F^{-1}$. From this combinatorial picture we see: deleting all edges named $F^{-1}$ in $\Gamma^{\prime}$ we obtain a connected graph, it contains exactly one cycle containing an odd number of symbols $\perp$, and it contains all symbols $V$ in $w$ and in $\bar{w}$. This proves (ii).

Suppose $\Gamma^{\prime}=\Gamma_{i} \neq \perp\left(\Gamma^{\prime}\right)=\Gamma^{\prime \prime}$ for some index $i$. Let $w$ be the shortest word in $V$ and $F^{-1}$ needed to go through $\Gamma^{\prime}$ once. Then $\bar{w}$ is the shortest word needed to go through $\Gamma^{\prime \prime}$ once. Every vertex of $\Gamma^{\prime}$ is connected to a unique vertex in $\Gamma^{\prime \prime}$ by $\perp$; every $V$-edge in $\Gamma^{\prime}$ corresponds to a unique $F^{-1}$-edge in $\Gamma^{\prime \prime}$. This proves that the $(V, \perp)$-equivalence class of any element in $\Gamma^{\prime} \cup \Gamma^{\prime \prime}$ equals $\Gamma^{\prime} \cup \Gamma^{\prime \prime}$, and it shows that there is precisely one $(V, \perp)$-cycle; this cycle has an even number of symbols $\perp$ in the cycle. This finishes the proof of the lemma.

(5.11) Notation. Let $\varphi$ be an elementary sequence, with associated canonical type $\tau$. We write $\mathcal{C}_{\varphi}()=\mathcal{D}_{\tau}()$. We write

$$
S_{\varphi}:=\mathcal{D}_{\tau}(\mathcal{A}) \subset \mathcal{A}:=\mathcal{A}_{g, 1, n} \otimes \mathbb{F}_{p, n},
$$

see (3.3), for the locally closed subset where the elementary sequence is constant and equal to $\varphi$.

\section{$6 \quad$ Strata at the boundary, strata in $\mathcal{A}_{g, 1}^{*} \otimes \mathbb{F}_{p}$}

(6.1) The elementary sequence of a semi-abelian variety. For the definition of a semi-abelian scheme $X \rightarrow S$ we refer to [10], Def. 2.3 on page 8 . 
In particular, every geometric fiber $X_{s}$ is an extension over an abelian variety with kernel $T_{s} \cong\left(\mathbb{G}_{m}\right)^{\beta}$ for some non-negative integer $\beta$.

For a semi-abelian variety $X$ over a field K of characteristic $p$ we define its $p$-rank $f=f(X)$ by:

$$
\operatorname{Hom}\left(\mu_{p}, X \otimes k\right) \cong(\mathbb{Z} / p)^{f},
$$

where $k$ is an algebraically closed field containing $K$. Note that if $X$ is an abelian variety, then $\#(X[p](k))=p^{f(X)}$.

Let $X$ be a semi-abelian variety over a field $K$; suppose that $L \subset X$ is the maximal connected linear subgroup; we write $Y=X / L$. We define the elementary sequence $\operatorname{ES}(X)=\varphi \in \Phi_{g}$ as follows. We consider $G:=X[p]$. This finite group has a filtration $0 \subset L[F]=G_{1} \subset X[F] \subset X[p]$; then $G_{1} \otimes k \cong\left(\mu_{p}\right)^{\beta}$, where $\beta:=\operatorname{dim}(L)$ and $X[p] / G_{1} \cong Y[p]$. We have a canonical filtration on $Y[p]$; this defines an elementary sequence $\theta:=\operatorname{ES}(Y) \in \Phi_{g-\beta}$. We define $\varphi=\operatorname{ES}(X)$ by: $\varphi(i)=i$ for all $0 \leq i \leq \beta$ and $\varphi(i)=\theta(i)+\beta$ for all $\beta \leq i \leq g$; in this way $\mathrm{ES}(X)$ is defined:

$$
\varphi=\{1, \cdots, \beta, \cdots, \theta(i)+\beta, \cdots\} .
$$

Here is another way of defining this sequence. Consider, as in (2.2), the set $E$ of all (finite) words in the symbols $V$ and $F^{-1}$. The set of such words defines on $N=X[p]$ a set of finite subgroup schemes $\{W(N) \mid W \in E\}$; this is a filtration; to this we associate a "canonical filtration" of $N$, a canonical type, and by truncation an elementary sequence. This is the one defined earlier in case $\beta=0$, i.e. $X$ is an abelian variety; it coincides with the elementary sequence just defined in all cases.

Suppose that $X_{1} / L_{1}=Y_{1}$ and $X_{2} / L_{2}=Y_{2}$ are semi-abelian varieties with $Y_{1} \cong Y_{2}$. Then $\operatorname{ES}\left(X_{1}\right)=\operatorname{ES}\left(X_{2}\right)$; this follows from the construction.

By $\mathcal{A}^{*}$ we denote the minimal compactification of $\mathcal{A}=\mathcal{A}_{g, 1, n} \otimes \mathbb{F}_{p, n}$, see [10], Chap. V, in particular see pp. 150, 152, Theorem 2.3 and Theorem 2.5. Note that a geometric point of $x^{\prime} \in \mathcal{A}^{*}$ defines an abelian variety $Y$ (with a principal polarization, with some level structure); hence the point $x^{\prime}$ defines an elementary sequence (by the data $Y$ and $g$ ). Let $\overline{\mathcal{A}} \rightarrow \mathcal{A}^{*}$ be a toroidal compactification, let $x$ be a geometric point of $\overline{\mathcal{A}}$, and $x \mapsto x^{\prime}$. The elementary sequence defined by $x$ only depends on $x^{\prime} \in \mathcal{A}^{*}$. Essential feature: $\operatorname{ES}(X)$ only depends on $Y=X / L$, and on $X[F]$ we can give a filtration (see below).

Let $\varphi$ be an elementary sequence. We write $T_{\varphi} \subset \mathcal{A}^{*}$ for the set of points $s \in \mathcal{A}^{*}$ with elementary sequence equal to $\varphi$.

(6.2) Proposition. The set $T_{\varphi} \subset \mathcal{A}^{*}$ is locally closed.

Proof. Let $\psi: \overline{\mathcal{A}} \rightarrow \mathcal{A}^{*}$ be a toroidal compactification and $T_{\varphi}^{\prime} \subset \overline{\mathcal{A}}$ be the set of points $x \in \overline{\mathcal{A}}$ with elementary sequence equal to $\varphi$. Then $\psi^{-1}\left(T_{\varphi}\right)=T_{\varphi}^{\prime}$; moreover $\psi$ is a proper morphism. Hence it suffices to prove that $T_{\varphi}^{\prime} \subset \overline{\mathcal{A}}$ is locally closed. 
It suffices to prove the proposition locally on $\overline{\mathcal{A}}$. For points $s \in T_{\varphi} \cap \mathcal{A}=$ $T_{\varphi}^{\prime} \cap \mathcal{A}=S_{\varphi} \subset \mathcal{A}$ this has already been proved. Let $s \in T_{\varphi}^{\prime} \cap \partial(\overline{\mathcal{A}})=\overline{\mathcal{A}}-\mathcal{A}$. We use results about "Mumford's uniformization theorem", and about "Raynaud extensions", see [10], in particular pp. 35/52, pp. 102 - 112 and pp. 149/150. By these theories there exist the following data:

- a complete local normal domain $R$, a semi-abelian scheme $\mathcal{X} \rightarrow S:=$ $\operatorname{Spec}(R)$ with generic fiber $X_{\eta}$ which is an abelian variety with a principal polarization $\lambda_{\eta}$, and a "Kodaira-Spencer" morphism $f: \operatorname{Spec}(R) \rightarrow \overline{\mathcal{A}}$ defined by this family such that the closed point $0 \in \operatorname{Spec}(R)$ maps to $f(0)=s$ and such that $R \hookleftarrow \mathcal{O}_{\bar{A}, s}$ is injective;

- an exact sequence $0 \rightarrow \mathcal{L} \rightarrow \mathcal{Z} \rightarrow \mathcal{Y} \rightarrow 0$ of group schemes over $S$, where $\mathcal{Z} \rightarrow S$ is a semi-abelian scheme, where $\mathcal{Y} \rightarrow S$ is an abelian scheme, with a principal polarization $\lambda^{\prime}$ on $\mathcal{Y} \rightarrow S$, and there exists an isomorphism $\mathcal{L} \cong\left(\mathbb{G}_{m}\right)^{\beta} \rightarrow S$ (actually there is more structure here involved, which however we do not need);

- these are related by the fact that the formal semi-abelian schemes derived from these two set of data are isomorphic: $\mathcal{X}^{\wedge} \cong_{\operatorname{Spf}(R)} \mathcal{Z}^{\wedge}$ and the isomorphism transforms the principal polarization induced by $\lambda$ into the abelian part of $\mathcal{X}_{0}$ with $\lambda_{0}^{\prime}$ on $Y_{0}=\mathcal{Y} \times_{S}\{0\}$.

Suppose $\varphi \in \Phi_{g}$ and $\theta \in \Phi_{g-\beta}$ are as above. Using [10], III.7, we see that the set of points $T=f^{-1}\left(T_{\varphi}^{\prime}\right) \subset S$ equals $\mathcal{C}_{\theta}(\mathcal{Y} \rightarrow S)$; by Proposition (3.2) this is locally closed in $S$. By assumption we have $0 \in T$. By $R \hookleftarrow \mathcal{O}_{\overline{\mathcal{A}}, s}$ and $s \in T_{\varphi} \cap \partial(\overline{\mathcal{A}})$ we conclude that $T_{\varphi}^{\prime} \subset \overline{\mathcal{A}}$ is closed at $s$. This finishes the proof.

(6.3) Proposition. Let $\varphi \in \Phi_{g}$. Let $Z$ be an irreducible component of $S_{\varphi}=$ $\mathcal{C}_{\varphi}(\mathcal{A})$, and let $Z^{*}$ be the closure of $Z \subset \mathcal{A}^{*}$. Suppose there is an elementary sequence $\varphi^{\prime} \in \Phi_{g}$ with $Z^{*} \cap T_{\varphi^{\prime}} \neq \emptyset$. Then the Zariski closure $Z^{c}$ of $Z \subset \mathcal{A}$ meets $S_{\varphi^{\prime}}$ :

$$
Z^{c} \cap S_{\varphi^{\prime}} \neq \emptyset .
$$

Let $\varphi^{\prime} \in \Phi$, and let $W$ be an irreducible component of $T_{\varphi^{\prime}}$. Then

$$
W \cap \mathcal{A} \neq \emptyset \text {. }
$$

In particular, every irreducible component of $T_{\varphi}$ meets $\mathcal{A}$, and intersects this interior into a component of $S_{\varphi}$.

Proof. The second and the third statement follow from the first.

As in the proof of the previous proposition it suffices to prove this result for a toroidal compactification $\psi: \overline{\mathcal{A}} \rightarrow \mathcal{A}^{*}$.

Let $s \in \bar{Z} \cap \partial(\overline{\mathcal{A}})$ and $s \in T_{\varphi^{\prime}}^{\prime}$. Using results on toroidal compactifications and on "Mumford's uniformization theorem", and about "Raynaud extensions", we conclude that we have the following data: 
- a noetherian complete domain $R$, which is complete for an ideal $I \subset R$, we write $S=\operatorname{Spec}(R)$, and $S_{0}=\operatorname{Spec}\left(R_{0}\right)$, we write $S^{\wedge}$ for the completion of $S$ along $S_{0}$ by the ideal $I$, a semi-abelian scheme $\mathcal{X} \rightarrow S$, with generic fiber $X_{\eta}$ which is an abelian variety with a principal polarization $\lambda_{\eta}$, and a "Kodaira-Spencer" morphism $f: \operatorname{Spec}(R) \rightarrow \overline{\mathcal{A}}$ defined by this family such that the closed point $0 \in \operatorname{Spec}(R)$ maps to $f(0)=s$, such that $R \hookleftarrow \mathcal{O}_{\overline{\mathcal{A}}, s}$, and a morphism $\pi: S \rightarrow \mathcal{A}^{*}$ obtained as the composition $S \rightarrow \mathcal{A}_{g-\beta, 1, n} \otimes \mathbb{F}_{p, n}=: \mathcal{A}^{\prime} \subset \partial(\mathcal{A}) \subset \mathcal{A}^{*} ;$ N.B. if $\beta>0$, we will see that the morphism $\pi$ in general does not factor $f$;

- an exact sequence $0 \rightarrow \mathcal{L} \rightarrow \mathcal{Z} \rightarrow \mathcal{Y} \rightarrow 0$ of group schemes over $S$, where $\mathcal{Z} \rightarrow S$ is a semi-abelian scheme, where $\mathcal{Y} \rightarrow S$ is an abelian scheme, with a principal polarization $\lambda^{\prime}$ on $\mathcal{Y} \rightarrow S$, and there exists an isomorphism $\mathcal{L} \cong\left(\mathbb{G}_{m}\right)^{\beta} \rightarrow S$, such that the "Kodaira-Spencer" morphism given by $\left(\mathcal{Y}, \lambda^{\prime}\right) \rightarrow S$ defines $\pi: S \rightarrow \mathcal{A}$

- these are related by the fact that the formal semi-abelian schemes derived from these two set of data are isomorphic: $\mathcal{X}^{\wedge} \widetilde{=}_{S_{0}} \mathcal{Z}^{\wedge}$, and the isomorphism transforms the principal polarization on the generic fiber of $\mathcal{X}$ into $\lambda^{\prime}$ on $\mathcal{Y} \rightarrow S$.

From this the proposition follows: consider $\bar{Z} \rightarrow \overline{\mathcal{A}} \leftarrow S$, and the fiber product $P$ of this diagram; there exists an irreducible component $U \subset \mathcal{C}_{\varphi}(S) \subset S$ such that its closure $Z^{* *}=U^{c} \subset\left(\mathcal{C}_{\varphi}(S)\right)^{c} \subset S$ is an irreducible component of $P$ containing $0 \in S$. Let $\theta$ be as before. We see that $\pi\left(Z^{* *}\right) \subset \pi\left(\left(\mathcal{C}_{\varphi}(S)\right)^{c}\right) \subset$ $\left(\mathcal{C}_{\theta}\left(\mathcal{A}^{\prime}\right)\right)^{c} \subset \mathcal{A}^{\prime}$, the last closures taken in $\mathcal{A}^{\prime}$. We see that there exists a complete discrete valuation $\operatorname{ring} \Lambda$, with $\Gamma=\operatorname{Spec}(\Lambda)$ and a morphism $h: \Gamma \rightarrow S$ such that $h(\Gamma) \subset Z^{* *} \cap \pi^{-1}(s)$ and $f(h(\Gamma)) \not \subset \partial(\mathcal{A})$. The generic point $a \in \Gamma$ has the property $\pi(h(a))=s \in T_{\varphi^{\prime}}^{\prime}$; because $h(\Gamma) \subset \pi^{-1}(s)$ we conclude that $\operatorname{ES}\left(\mathcal{X}_{h(a)}\right)=\operatorname{ES}\left(X_{0}\right)=\varphi^{\prime}$. Moreover $h(a)$ is in the closure of $U$, hence $f(h(a)) \in \bar{Z}$. Hence $\bar{Z} \cap S_{\varphi^{\prime}} \neq \emptyset$.

(6.4) Proposition. Let $S$ be an irreducible, reduced, normal, noetherian scheme over $\mathbb{F}_{p}$, and let $X \rightarrow S$ be a semi-abelian scheme, with generic fiber an abelian variety with a principal polarization. Suppose all fibers have the same elementary sequence $\varphi \in \Phi_{g}$, i.e. the moduli map $S \rightarrow \mathcal{A}^{*}$ maps $S$ into $T_{\varphi}$. Consider $X[p] \rightarrow S$. There exists a filtration $0 \subset U \subset M \subset X[p]$ with finite group schemes $U$ and $M$ flat over $S$, such that $U \rightarrow S$ is of multiplicative type over $S$ in every fiber, and such that for every point $s \in S$, the fiber $N_{s}:=M_{s} / U_{s}$ is of local-local type, and every fiber $X[p]_{s} / M_{s}$ is étale-local. The group schemes $U \rightarrow S$ and $M \rightarrow S$ are flat over $S$. The finite group scheme $M / U=: N \rightarrow S$ is flat over $S$, and it is symmetric; it admits a canonical flltration and it satisfies the properties for the canonical filtration as described in Corollary (2.6). 
Remark. In general, the rank of $X[p]_{s} / M_{s}$ is not constant on $S$.

Proof. The finite group schemes $U$ and $M$ defined by the properties mentioned have constant rank (all geometric fibers have the same rank) because of the fact that the elementary sequence is constant on $S$. Moreover note that for every $j \in \mathbb{Z}_{>0}$ the finite group scheme $X\left[F^{j}\right] \rightarrow S$ is flat, and $U \subset M \subset X\left[F^{N}\right]$ are closed subgroup schemes, hence finite, over $S$ for large $N$ (e.g. $N=g$ ). As the elementary sequence of $X \rightarrow S$ is constant on $S$, we conclude that the finite group schemes $U \rightarrow S$ and $M \rightarrow S$ have constant rank, and hence these are flat by [4], Lemma 1.13 on page 240 . The principal polarization on the generic fiber extends to a symmetry on $N=M / U$, e.g. use [10], Proposition I.2.7. The rest of the proof is as in Section 2, especially as in the proof of (2.6).

(6.5) Theorem. For every $\varphi \in \Phi_{g}$ the stratum $T_{\varphi} \subset \mathcal{A}^{*}$ is quasi-affine.

Proof. It suffices to show this in case of arbitrary high level structure. Hence we suppose $\mathcal{A}^{*}=\mathcal{A}_{g, 1, n}^{*} \otimes \mathbb{F}_{p, n}$, with $n \in \mathbb{Z}_{>3}$ not divisible by $p$. It suffices to show that every irreducible component $V \subset \bar{T}_{\varphi} \subset \mathcal{A}^{*}$ is quasi-affine. We choose such a component $V$. Note that we know that $V$ meets the interior of $\mathcal{A}^{*}$, see (6.3). We proceed as in the proof of (4.1).

By results in [10], V.2, there exist an irreducible, normal, reduced scheme $S \rightarrow$ $\operatorname{Spec}\left(\mathbb{F}_{p}\right)$, a semi-abelian scheme $\mathcal{X} \rightarrow S$, a principal polarization on its generic fiber which is an abelian variety, and a symplectic level- $n$-structure, such that the moduli morphism $S \rightarrow \mathcal{A}^{*}$ defined by these data factors through $V$, and such that $f: S \rightarrow V$ is proper and surjective. We write $\mathfrak{t}_{\mathcal{X} / S}$ for the tangential sheaf of $\mathcal{X} \rightarrow S$ along the zero-section, and we write $\omega_{\mathcal{X} / S}$ for the determinant line bundle of the dual of $t_{\mathcal{X} / S}$, see [10], page 137. We know, see [10], V.2.5, that the moduli morphism $S \rightarrow \mathcal{A}^{*}$ is defined by the line bundle $\omega_{\mathcal{X}} / S$.

Claims. 1) Under these conditions, the line bundle $\omega_{X / S}$ on $S$ is a torsion line bundle.

2) From this it follows that $V$ is quasi-affine.

Indeed, suppose (1) is proven and let us see that the result is established; consider the Stein factorization $S \rightarrow V^{\prime} \rightarrow V$, as in EGA II, 1.3.1; then $f^{\prime}$ : $S \rightarrow V^{\prime}$ is proper with connected fibers, and $V^{\prime} \rightarrow V$ is finite. By EGA III, 4.3.1 we know that $f_{*}^{\prime}\left(\mathcal{O}_{S}\right) \cong \mathcal{O}_{V^{\prime}}$. Because $V^{\prime} \rightarrow V$ is finite, assuming (1), it follows that $f_{*}\left(\omega_{\mathcal{X} / S}\right)$ is torsion on $V$. Using [35], Chap. IX, and [10], V.2, Th. 2.3 , we conclude that $\left.\omega\right|_{V}$ is ample and torsion; hence $V$ is quasi-affine. This proves $(1) \Rightarrow(2)$.

We show that (1) is true. Let $\gamma$ be the canonical type associated with $\varphi$, say $\gamma=(\nu, \rho)$. We write $f=f(\gamma)$, i.e. the largest index with $\varphi(f)=f$, e.g. $f=0$ if $\nu(1)=0$, and $f=\rho(1)$ if $\nu(1)=1$. We write $U=0$ if $f=0$, and we write $U=G_{1}$ if $f>0$. We use Proposition (5.9), we use that the elementary 
sequence on $\mathcal{X} \rightarrow S$ is constant, we use [4], Lemma 1.13 on page 240 , and we conclude that we have finite flat subgroup schemes

$$
0=G_{0} \subset G_{1}=U \subset G_{2} \subset \cdots \subset G_{r}=G[F]=V(G[p])
$$

where $G_{1} \times{ }_{S} S^{\prime} \cong\left(\mu_{p}\right)^{f} \times S^{\prime}$ for some finite cover $S^{\prime} \rightarrow S$, and where the other steps in the filtration come from the canonical filtration on $M / U=N$, with the notation as in (5.9). Note that the partial quotients $G_{i} / G_{i-1}$ are annihilated by $F$ and by $V$ for $1<i \leq r$ (in the terminology of [29], 2.4, these are $\alpha$-groups). We see that all tangential sheaves

$$
\mathfrak{t}_{G_{1} / S} \subset \cdots \subset \mathfrak{t}_{G_{r} / S}=\mathfrak{t}_{\mathcal{X} / S}
$$

are well-defined, of constant rank. We write $\mathcal{L}_{i}:=\operatorname{Det}\left(\mathfrak{t}_{G_{i} / S} / \mathfrak{t}_{G_{i-1} / S}\right)$, for $0<$ $i \leq r$, and we are going to show that these are torsion line bundles.

This is clear for the first step in the filtration: over $S^{\prime}$ as above the pullback of $\mathcal{L}_{1}$ is trivial, hence its norm is trivial, which shows that $\left(\mathcal{L}_{1}\right)^{\text {degree }\left(S^{\prime} / S\right)}=$ 1 .

Consider one of the other steps $G_{j} / G_{j-1}$ with $j>1$. We apply Corollary (2.6). In case

$$
\left(G_{j} / G_{j-1}\right)^{\left(p^{n}\right)} \stackrel{\sim}{\longrightarrow} G_{j} / G_{j-1}
$$

we get

$$
\left(\mathcal{L}_{j}\right)^{p^{n}}=\left(\mathcal{L}_{j}\right)
$$

in this case this shows the class to be torsion (note that $n>0$ ).

We conclude that $\operatorname{Det}\left(\mathfrak{t}_{\mathcal{X} / S}\right)=\operatorname{Det}\left(\mathcal{L}_{1}\right) \otimes \cdots \otimes \operatorname{Det}\left(\mathcal{L}_{r}\right)$ is torsion; hence its inverse $\omega_{\mathcal{X} / S}$ is torsion. This shows (1), and it finishes the proof of Theorem $(6.5)$.

\section{Connecting the superspecial locus inside the supersingular locus}

In this section we construct a complete algebraic curve $L \subset \mathcal{A}$, with $\Sigma \subset L$. We write $L^{\circ}=L-\Sigma$. We show that $L^{\circ} \subset S_{\{0, \cdots 0,1\}}$, and in Section 8 we show equality. An essential tool in the rest of the paper will be the property that $L$ is connected, see Proposition (7.3). Actually, the curve $L$ is contained in the supersingular locus of $\mathcal{A}$ and the proof of (7.3) uses pure algebra. The basic idea of this section is due to T. Ekedahl. The present form of this section is mainly due to Ben Moonen who helped with most details in this section.

In case $g=1$ we will write $L=\mathcal{A}$ and $L^{\circ}=L-\Sigma$. In this section we consider abelian varieties of dimension $g \geq 2$. 
(7.1) Construction of $L$. Let $k$ be an algebraically closed field of characteristic $p$. Choose a point $x \in \Sigma(k)$; let $(X, \mu, \eta)$ be the corresponding principally polarized abelian variety with symplectic level- $n$-structure.

For the construction of the locus $L$ we are interested in coverings $f: Y \rightarrow X$ such that

(i) $\operatorname{Ker}(f) \cong \alpha_{p}$,

(ii) $Y$ is superspecial,

(iii) $\operatorname{Ker}\left(f^{*} \mu\right) \cong \alpha_{p} \times \alpha_{p}$.

Coverings $f$ satisfying (i) correspond (by duality) to subgroup schemes of $X$ isomorphic to $\alpha_{p}$. Such subgroup schemes are parameterized by the projective space $\mathbb{P}(\operatorname{Lie}(X)) \cong \mathbb{P}^{g-1}$. One can show, see [34], pp. 138/139, [21], Prop. 5.2, that the coverings $f$ satisfying (i) and (ii) are in bijection with $\mathbb{P}^{g-1}\left(\mathbb{F}_{p^{2}}\right)$, and that the coverings satisfying (i)-(iii) correspond to those vectors in $\mathbb{P}^{g-1}\left(\mathbb{F}_{p^{2}}\right)$, "very good directions", which are isotropic with respect to a certain hermitian form.

Given a covering $f: Y \rightarrow X$ satisfying (i)-(iii), write $t$ for the tangent space of $\operatorname{Ker}\left(f^{*} \mu\right)$; this is a 2 -dimensional $k$-vector space. A point $s \in \mathbb{P}(\mathfrak{t}) \cong \mathbb{P}^{1}$ corresponds uniquely to a subgroup scheme $\alpha_{p} \cong N=$ $N_{s} \subset \operatorname{Ker}\left(f^{*} \mu\right) \subset Y[p]$, and conversely every such subgroup scheme gives a point in $\mathbb{P}(\mathfrak{t})$. Moreover every such subgroup scheme is totally isotropic for the pairing induced on $\operatorname{Ker}\left(f^{*} \mu\right)$; it follows from condition (iii) that for every such $N$, the polarization $f^{*} \mu$ descends to a principal polarization $\mu_{s}$ on the quotient $X_{s}:=Y / N_{s}$. Further, as we have natural isomorphisms $X_{s}[n] \stackrel{\leftarrow}{\leftarrow} Y[n] \stackrel{\sim}{\rightarrow} X[n]$, the symplectic level-n-structure $\eta$ of $(X, \mu)$ induces a symplectic level-n-structure $\eta_{s}$ of $\left(X_{s}, \mu_{s}\right)$. Taking $s$ to be the point corresponding to $\operatorname{Ker}(f) \subset \operatorname{Ker}\left(f^{*} \mu\right)$ gives $\left(X_{s}, \mu_{s}, \eta_{s}\right)=(X, \mu, \eta)$.

In total, the choice of $x \in \Sigma(k)$ and the covering $f$ gives rise to a nonconstant morphism $\mathbb{P}_{k}^{1} \cong \mathbb{P}(\mathfrak{t}) \longrightarrow \mathcal{A}_{k}=\mathcal{A}_{g, 1, n} \otimes k$; the image is denoted by $L^{(f)}$. We define $L_{k} \subset \mathcal{A}_{k}$ to be the reduced closed subscheme which is the union of the images of all morphisms thus obtained (varying $x$ and $f$ ), $L_{k}=\cup_{f} L^{(f)}$. One can show that the closed subscheme $L_{k}$ is defined over $\mathbb{F}_{p}$, i.e., there is a closed subscheme $L \subset \mathcal{A}_{g, 1, n}$ such that $L_{k}=L \otimes k$.

We indicate a different construction of $L$, and we derive some properties. We write $H:=G_{1,1}$ for the formal $p$-divisible group of dimension one and height 2. For a supersingular elliptic curve $E$ over $k$ we have $E\left[p^{\infty}\right] \cong_{k} H \otimes k$.

(7.2) Lemma. A homomorphism $f: Y \rightarrow X$ as above can be constructed by a choice $X\left[p^{\infty}\right] \cong H^{2} \oplus H^{g-2}$ such that $\mu$ is in block form $\mu_{2} \oplus\left(\mu_{1}\right)^{g-2}$, correspondingly $Y\left[p^{\infty}\right] \cong H^{2} \oplus H^{g-2}$ and

$$
\operatorname{Ker}\left(f^{*}(\mu)\right) \subset H^{2} \subset H^{2} \oplus H^{g-2} .
$$


We have $\Sigma \subset L \subset\left(S_{\{0, \ldots, 0,1\}}\right)^{c}$.

Proof. For the superspecial $Y$ we have $Y\left[p^{\infty}\right] \cong H^{g}$. By [29], 6.1 we see that the polarization $f^{*}(\mu)$ on $Y$ gives a quasi-polarization on $Y\left[p^{\infty}\right] \cong H^{g} \cong M_{1} \oplus$ $M_{2} \oplus \cdots \oplus M_{g-1}$ in block-form with $M_{1} \cong H^{2}$, with $\operatorname{Ker}\left(f^{*}(\mu)\right) \subset M_{1} \cong H^{2}$ and $M_{j} \cong H$ for $2 \leq j \leq g-1$. This proves the first claim.

Suppose $s \in \mathbb{P}(\mathfrak{t})$, with $X_{s}:=Y / N_{s}$. Choose coordinates on $\mathbb{P}(\mathfrak{t}) \cong \mathbb{P}^{1}$ by $M_{1} \cong H^{2}$. We know that $a\left(X_{s}\right)=1$ iff $s \notin \mathbb{P}^{1}\left(\mathbb{F}_{p^{2}}\right)$; suppose this is the case; then $X_{s}[p]:=N \cong N_{1} \oplus N_{2} \oplus \cdots \oplus N_{g-1}$ with $\operatorname{rk}\left(N_{1}\right)=p^{4}$ and $\operatorname{ES}\left(N_{1}\right)=\{0,1\}$ and for $2 \leq j \leq g-1$ we have $\operatorname{rk}\left(N_{j}\right)=p^{2}$ and $\operatorname{ES}\left(N_{j}\right)=\{0\}$. Direct verification shows that in this case $\operatorname{ES}\left(X_{s}\right)=\{0, \ldots, 0,1\}$. As we see that $\Sigma \subset L$, all components of $L$ have dimension one, and $L^{\circ}:=L-\Sigma \subset S_{\{0, \ldots, 0,1\}}$ the second claim follows.

Conclusion. Every component $L^{(f)} \subset L_{k}$ as in (7.1) can be given by choosing $[(X, \mu)] \in \Sigma(k)$, by choosing an isomorphism $X\left[p^{\infty}\right] \cong H^{g}$ on which $\mu$ is in diagonal form $\operatorname{diag}(\lambda, \cdots, \lambda)$, see [29], 6.1, by choosing two factors: $H^{g}=H^{2} \oplus H^{g-2}$, and by performing a construction of type [34], pp. 138/138, on $\left(H^{2}, \operatorname{diag}(\lambda, \lambda)\right)$.

Remark. The fact that $S_{\{0, \cdots, 0\}}=\Sigma \subset\left(S_{\{0, \cdots, 0,1\}}\right)^{c}$ is a special case of (11.1).

(7.3) Proposition. The locus $L \subset \mathcal{A}$ is 1-dimensional and connected.

(7.4) Remark. In Section 8 we shall show that in fact $L=\left(S_{\{0, \ldots, 0,1\}}\right)^{c}$. In this section we shall prove the proposition as stated here. For this we need a number of preparations about hermitian forms, which will be given in (7.5) (7.11). The proof of (7.3) is given in (7.18) below.

(7.5) Let $R$ be a ring equipped with an (anti-)involution $r \mapsto r^{\dagger}$. Let $M$ be a finitely generated projective right $R$-module. By a hermitian form on $M$ (with respect to the involution $\dagger$ ) we mean a bi-additive map

$$
\psi: M \times M \longrightarrow R
$$

such that

(a) $\psi$ is sesquilinear, meaning that $\psi\left(m_{1} r_{1}, m_{2} r_{2}\right)=r_{1}^{\dagger} \cdot \psi\left(m_{1}, m_{2}\right) \cdot r_{2}$ for all $r_{1}, r_{2} \in R$ and $m_{1}, m_{2} \in M$;

(b) $\psi\left(m^{\prime}, m\right)=\psi\left(m, m^{\prime}\right)^{\dagger}$ for all $m, m^{\prime} \in M$.

We say that a form $\psi$ as above is skew-hermitian if it satisfies (a) and:

(b') $\psi\left(m^{\prime}, m\right)=-\psi\left(m, m^{\prime}\right)^{\dagger}$ for all $m, m^{\prime} \in M$. 
The dual $M^{\vee}:=\operatorname{Hom}_{R}(M, R)$ of $M$ has a natural structure of a left $R$-module. Let $M^{\dagger}:=M^{\vee}$ as an additive group, and give it the structure of a right $R$ module by $f r:=r^{\dagger} \cdot f$. A form $\psi$ satisfying (a) gives rise to a homomorphism $M \rightarrow M^{\dagger}$ by $m \mapsto \psi(m,-)$. If this map is an isomorphism we say that $\psi$ is perfect.

By a hermitian space over $R$ we mean a pair $(M, \psi)$ consisting of a projective right $R$-module of finite type equipped with a perfect hermitian form $\psi$.

(7.6) Let $P$ be a field. Write $A \mapsto A^{\dagger}=\operatorname{Trd}(A)-A$ for the canonical involution on the algebra $M_{2}(P)$ of $2 \times 2$ matrices over $P$. Consider $V_{1}:=P^{2}$ with its natural structure of a right $M_{2}(P)$-module. Up to a scalar in $P^{\times}$there is a unique perfect skew-hermitian form on $V_{1}$ with respect to $\dagger$, namely the form $\Psi_{1}: V_{1} \times V_{1} \longrightarrow M_{2}(P)$ given by

$$
\Psi_{1}\left((x, y),\left(x^{\prime}, y^{\prime}\right)\right)=\left(\begin{array}{cc}
y x^{\prime} & y y^{\prime} \\
-x x^{\prime} & -x y^{\prime}
\end{array}\right) .
$$

Let $\left(V_{2}, \Psi_{2}\right)$ be a symplectic space over $P$, by which we mean a finite dimensional $P$-vector space $V_{2}$ with a non-degenerate alternating bilinear form $\Psi_{2}: V_{2} \times V_{2} \longrightarrow P$. The dimension of $V_{2}$ is even, say $\operatorname{dim}_{P}\left(V_{2}\right)=2 g$. Then $V:=V_{1} \otimes_{P} V_{2}$ becomes a free right $M_{2}(P)$-module of rank $g$ via the action of $M_{2}(P)$ on $V_{1}$, and the tensor product $\Psi_{1} \otimes_{P} \Psi_{2}$ is a perfect hermitian form on $M_{1} \otimes P M_{2}$.

Morita equivalence tells us that every hermitian space $(V, \Psi)$ of $\operatorname{rank} g$ over $M_{2}(P)$ (with respect to the canonical involution) is isometric to such a tensor product, see [25], I.9. In particular, since any two symplectic spaces of the same dimension over $P$ are isometric, also all hermitian spaces of the same rank over $M_{2}(P)$ are isometric (also see: [8], Section 3).

(7.7) Let $D$ be a quaternion algebra over $\mathbb{Q}$. Write $d \mapsto d^{\dagger}$ for its canonical involution. Fix a positive integer $g$. Let $(V, \Psi)$ be a hermitian space of rank $g$ over $D$. Then

$$
G:=\operatorname{Aut}_{D}(V, \Psi)
$$

is a linear algebraic group over $\mathbb{Q}$. It is immediate from what was explained in (4.4) that if $\mathbb{Q} \subset L$ is a field extension which splits $D$ then $G \otimes_{\mathbb{Q}} L \cong$ $\mathrm{Sp}_{2 g, L}$. In particular, $G$ is absolutely simple and simply connected. Assume that $D_{\mathbb{R}}:=D \otimes \mathbb{Q}$ is non-split. Write $\Psi_{\mathbb{R}}$ for the $\mathbb{R}$-bilinear extension of $\Psi$ to a hermitian form on $V_{\mathbb{R}}$. Define $q: V_{\mathbb{R}} \rightarrow \mathbb{R}$ by $q(v)=\Psi_{\mathbb{R}}(v, v)$; note that it follows from (b) in (7.5) that indeed $\Psi_{\mathbb{R}}(v, v) \in \mathbb{R} \subset D_{\mathbb{R}}$. Then $q$ is a quadratic form on $V_{\mathbb{R}}$. The signature of $\Psi$ is defined to be $\operatorname{sign}(\Psi):=\operatorname{sign}(q) / 4$. Two hermitian spaces $(V, \Psi)$ and $\left(V^{\prime}, \Psi^{\prime}\right)$ over $D$ are isometric if and only if $\operatorname{dim}(V)=\operatorname{dim}(V)$ and $\operatorname{sign}(\Psi)=\operatorname{sign}\left(\Psi^{\prime}\right)$, see for instance [53], Chapter $10, \S 1$. We say that $\Psi$ is positive definite if $q$ is; this is equivalent to $\operatorname{sign}(\Psi)=g$. 
(7.8) With $(D, \dagger)$ as in (7.7), fix a hermitian space $(V, \Psi)$ of rank $g$ over $D$. Let $R \subset D$ be an order; note that $R$ is stable under the involution $\dagger$. Let $(M, \psi)$ be a hermitian space of rank $g$ over $R$ such that there exists an isometry

$$
\alpha:\left(M_{\mathbb{Q}}, \psi_{\mathbb{Q}}\right) \stackrel{\sim}{\rightarrow}(V, \Psi) .
$$

Then $\alpha(M) \subset V$ is an $R$-lattice in $V$ on which $\Psi$ induces a perfect hermitian form; we shall refer to such lattices as perfect hermitian $R$-lattices in $V$. Note that $R$ is a hereditary ring; hence every $R$-lattice $M \subset V$ is projective, and if $\Psi_{\mid M}$ is perfect then $\left(M, \Psi_{\mid M}\right)$ is a hermitian space.

Let $n$ be a positive integer. Let us write $H_{n}$ for the standard hermitian form on $(R / n R)^{g}$, i.e., the form given by

$$
H_{n}\left(\left(x_{1}, \ldots, x_{g}\right),\left(y_{1}, \ldots, y_{g}\right)\right)=x_{1}^{\dagger} y_{1}+\cdots+x_{g}^{\dagger} y_{g} .
$$

If $(M, \psi)$ is a hermitian space of rank $g$ over $R$ then by a level- $n$-marking of $(M, \psi)$ we shall mean an isometry

$$
\eta:(M / n M, \bar{\psi}) \stackrel{\sim}{\rightarrow}\left((R / n R)^{g}, H_{n}\right),
$$

where we write $\bar{\psi}$ for the hermitian form on $M / n M$ induced by $\psi$.

(7.9) Lemma. Let $R$ be an order in a quaternion algebra $D$ over $\mathbb{Q}$. Let $p$ be a prime number, $n$ a positive integer prime to $p$. Let $g$ be an integer $\geq 2$, and fix a hermitian space $(V, \Psi)$ of rank $g$ over $D$ (with respect to the canonical involution on $D)$.

Let $\left(M_{1}, \psi_{1}, \eta_{1}\right)$ and $\left(M_{2}, \psi_{2}, \eta_{2}\right)$ be hermitian spaces of rank $g$ over $R$ equipped with level-n-markings. Assume that $\left(M_{j, \mathbb{Q}}, \psi_{j, \mathbb{Q}}\right)$ is isometric to $(V, \Psi)$, for $j=1,2$. Then there exist isometries

$$
\alpha_{j}:\left(M_{j, \mathbb{Q}}, \psi_{j, \mathbb{Q}}\right) \stackrel{\sim}{\rightarrow}(V, \Psi)
$$

such that $\alpha_{1}\left(M_{1}\right) \cap \alpha_{2}\left(M_{2}\right)$ has a p-power index in both $\alpha_{1}\left(M_{1}\right)$ and $\alpha_{2}\left(M_{2}\right)$, and such that via the isomorphism $M_{1} / n M_{1} \stackrel{\sim}{\rightarrow} M_{2} / n M_{2}$ induced by $\alpha_{2}^{-1} \circ \alpha_{1}$ the level-n-markings $\eta_{1}$ and $\eta_{2}$ correspond.

Note that if $\alpha_{1}$ and $\alpha_{2}$ satisfy the first condition then indeed $\alpha_{2}^{-1} \circ \alpha_{1}$ induces an isomorphism $M_{1} / n M_{1} \stackrel{\sim}{\rightarrow} M_{2} / n M_{2}$.

Proof. As above, write $G:=\operatorname{Aut}_{D}(V, \Psi)$. We claim that $G\left(\mathbb{Q}_{p}\right)$ is non-compact; this is equivalent to saying that $V \otimes \mathbb{Q}_{p}$ has an isotropic vector with respect to $\Psi$. If $D \otimes \mathbb{Q}_{\mathbb{Q}_{p}} \cong M_{2}\left(\mathbb{Q}_{p}\right)$ then the discussion in (7.6) shows that $G_{\mathbb{Q}_{p}}$ is split, and the claim is clear. Next suppose that $D_{p}:=D \otimes_{\mathbb{Q}} \mathbb{Q}_{p}$ is the (unique) quaternion algebra over $\mathbb{Q}_{p}$. Because $g \geq 2$ there exist two non-zero vectors $v_{1}, v_{2} \in V \otimes \mathbb{Q}_{p}$ with $\Psi\left(v_{1}, v_{2}\right)=0$. Now we use that the reduced norm map $D_{p}^{\times} \rightarrow \mathbb{Q}_{p}^{\times}$is surjective; using this it easily follows that some linear combination $v_{1} d_{1}+v_{2} d_{2}$ is isotropic. 
Write $\hat{\mathbb{Z}}^{(p)}:=\prod_{\ell \neq p} \mathbb{Z}_{\ell}$, where the product runs over all prime numbers $\ell$ different from $p$. Write $\mathbb{A}_{f}^{(p)}=\hat{\mathbb{Z}}^{(p)} \otimes_{\mathbb{Z}} \mathbb{Q}$ for the ring of finite adèles of $\mathbb{Q}$ without component at $p$. As $G\left(\mathbb{Q}_{p}\right)$ is non-compact and $G$ is absolutely simple and simply connected, we can apply strong approximation, see for example [49], Th. 7.12 on p. 427 , or see [24], see [51]. This tells us that the diagonal image of $G(\mathbb{Q})$ in $G\left(\mathbb{A}_{f}^{(p)}\right)$ is dense in the adèlic topology.

For $j=1,2$, write $\hat{M}_{j}^{(p)}:=M_{j} \otimes_{\mathbb{Z}} \hat{\mathbb{Z}}^{(p)}$. Choose isometries $\beta_{j}:\left(M_{j, \mathbb{Q}}, \psi_{j, \mathbb{Q}}\right) \stackrel{\sim}{\rightarrow}(V, \Psi)$. Write

$$
\tilde{\beta}_{j}: \hat{M}_{j}^{(p)} \otimes_{\hat{\mathbb{Z}}(p)} \mathbb{A}_{f}^{(p)} \stackrel{\sim}{\rightarrow} V \otimes_{\mathbb{Q}} \mathbb{A}_{f}^{(p)}
$$

for the $\mathbb{A}_{f}^{(p)}$-linear extension of $\beta_{j}$. Consider the set $\Gamma$ of all elements $\gamma \in G\left(\mathbb{A}_{f}^{(p)}\right)$ such that

$$
\left(\gamma \circ \tilde{\beta}_{1}\right)\left(\hat{M}_{1}^{(p)}\right)=\tilde{\beta}_{2}\left(\hat{M}_{2}^{(p)}\right)
$$

inside $V \otimes \mathbb{A}_{f}^{(p)}$. For $\gamma \in \Gamma$, write $t_{\gamma}:\left(M_{1} / n M_{1}\right) \stackrel{\sim}{\rightarrow}\left(M_{2} / n M_{2}\right)$ for the isomorphism induced by $\tilde{\beta}_{2}^{-1} \circ\left(\gamma \circ \tilde{\beta}_{1}\right)$. Let $\Gamma^{\prime} \subset \Gamma$ be the subset of all $\gamma$ which are compatible with the given level-n-markings, i.e.,

$$
\Gamma^{\prime}:=\left\{g \in \Gamma \mid \eta_{1}=\eta_{2} \circ t_{\gamma}\right\} .
$$

Then $\Gamma^{\prime}$ is a non-empty open subset of $G\left(\hat{A}_{f}^{(p)}\right)$. Hence it follows from strong approximation that there exists an element $g \in G(\mathbb{Q})$ which maps into $\Gamma$ under the diagonal embedding $G(\mathbb{Q}) \hookrightarrow G\left(\mathbb{A}_{f}^{(p)}\right)$. Then $\alpha_{1}:=g \circ \beta_{1}$ and $\alpha_{2}:=\beta_{2}$ are isometries with the required property.

(7.10) Keep the notations of (7.9). Assume that $D$ is inert at $p$. There is a unique 2-sided prime ideal $\mathfrak{p} \subset R$ above $p$, which is in fact a maximal ideal (both left and right). The assumption that $D$ is inert at $p$ implies that $k(\mathfrak{p}):=R / \mathfrak{p}$ is a field with $p^{2}$ elements, see [52], Th. 22.4 and Th. 22.14. The involution $\dagger$ on $R$ induces the unique non-trivial automorphism $\operatorname{Frob}_{k(\mathfrak{p})}$ of $k(\mathfrak{p})$.

Given an $R$-submodule $L \subset V$, write

$$
\breve{L}:=\{v \in V \mid \Psi(v, y) \in R \text { for all } y \in L\} .
$$

In particular, if $M \subset V$ is an $R$-lattice then $M=\breve{M}$ if and only if $\Psi$ induces a perfect form on $M$.

(7.11) Lemma Let $M$ and $M^{\prime}$ be perfect hermitian R-lattices in $V$ such that $M \cap M^{\prime}$ has p-power index in both $M$ and $M^{\prime}$. Assume that $\mathfrak{p} \subset R$ is a principal ideal. Then there exist perfect hermitian R-lattices

$$
M=L_{0}, L_{1}, \cdots, L_{m}=M^{\prime}
$$


such that $L_{j} /\left(L_{j} \cap L_{j+1}\right) \cong k(\mathfrak{p}) \cong L_{j+1} /\left(L_{j} \cap L_{j+1}\right)$ as $R$-modules for $0 \leq j<$ $m$.

Proof. Write $N:=M \cap M^{\prime}$. We claim that $\operatorname{length}_{R}(M / N)=\operatorname{length}_{R}\left(M^{\prime} / N\right)$. To see this, note that $\operatorname{Hom}_{R}(M / N, R)=0$ and that $\operatorname{Ext}_{R}^{1}(M, R)=0$. Hence we obtain an exact sequence

$$
0 \rightarrow M^{\dagger} \longrightarrow N^{\dagger} \longrightarrow \operatorname{Ext}_{R}^{1}(M / N, R) \rightarrow 0
$$

Now $M / N \cong R / \mathfrak{p}^{d_{1}} \oplus \cdots \oplus R / \mathfrak{p}^{d_{r}}$ as an $R$-module, in which case length $_{R}(M / N)=d_{1}+\cdots+d_{r}$. But if $\mathfrak{p}=(\pi)$ then we easily find that $\operatorname{Ext}_{R}^{1}\left(R / \mathfrak{p}^{d}, R\right) \cong R / \mathfrak{p}^{d}$. Hence $\check{N} / \check{M}=\check{N} / M \cong N^{\dagger} / M^{\dagger}$ has the same length as $M / N$. Doing the same with $M^{\prime}$ instead of $M$ we find that

$$
\operatorname{length}_{R}(\check{N} / N)=\operatorname{length}_{R}(M / N)^{2}=\operatorname{length}_{R}\left(M^{\prime} / N\right)^{2}
$$

which proves our claim.

We prove the lemma by induction on length ${ }_{R}(M / N)$. By what we have just shown, length $(M / N)=0$ corresponds to the case $M=M^{\prime}$. Note that $M+M^{\prime}=\check{N}$. On $\check{N} / N$ we have a hermitian form

$$
\chi: \check{N} / N \times \check{N} / N \longrightarrow D / R
$$

induced by $\Psi$. The subspaces $M / N$ and $M^{\prime} / N$ are maximal isotropic with respect to $\chi$.

Let $T \subset M / N$ be an $R$-submodule with $M / T \cong k(\mathfrak{p})$. Let $T^{\perp}$ be the orthogonal complement of $T$ with respect to $\chi$, set $T^{\prime}:=T^{\perp} \cap\left(M^{\prime} / N\right)$, and let

$$
S:=T+T^{\prime} \subset \check{N} / N \text {. }
$$

An easy calculation shows that $S$ is maximal isotropic for $\chi$. Hence the preimage $L \subset \breve{N}$ of $S$ is a perfect hermitian lattice in $V$. Now note that, by construction,

$$
\operatorname{length}_{R}\left(L /\left(L \cap M^{\prime}\right)\right)=\operatorname{length}_{R}(T)<\operatorname{length}_{R}(M / N) .
$$

Hence we can take $L_{1}=L$ and proceed by induction.

(7.12) Let $k$ be an algebraically closed field of characteristic $p$. Fix a supersingular elliptic curve $E$ over $k$. Then $E$ has a unique principal polarization, which we call $\lambda$. The endomorphism algebra $\operatorname{End}^{0}(E)$ is the quaternion algebra $Q_{p, \infty}$ over $\mathbb{Q}$ which is inert exactly at $p$ and $\infty$. The endomorphism ring $R:=\operatorname{End}(E)$ is a maximal order of this quaternion algebra. The Rosati involution $r \mapsto r^{\dagger}$ on $\operatorname{End}^{0}(E)$ associated to $\lambda$ (or to any other polarization) is the principal involution, i.e., $r^{\dagger}=\operatorname{Trd}(r)-r$. 
Let $X$ be a superspecial abelian variety of dimension $g \geq 2$ over $k$. This means that $X$ is isomorphic to $E^{g}$ as an abelian variety. Write

$$
M:=\operatorname{Hom}(E, X) \text {, }
$$

which has a natural structure of a free right $R$-module of rank $g$. We have a canonical isomorphism $M \otimes_{R} E \cong X$.

Note that $E^{t}$ has a natural structure of a right $R$-module. Similar to the definition of $M^{\dagger}$ in (4.3), let us write $E^{\dagger}$ for the elliptic curve $E^{t}$ with it structure of a left $R$-module given by $P r:=r^{\dagger} \cdot P$. Then $X^{t}$ is naturally isomorphic to $M^{\dagger} \otimes_{R} E^{\dagger}$.

(7.13) Remark. For any abelian variety $X$ over $k$, we can form the right $R$-module $\operatorname{Hom}(E, X)$. Conversely, for any right $R$-module $N$ of finite type, $N \otimes_{R} E$ is a supersingular abelian variety; to see this, take a finite presentation of $N$; we shall see that in this case $X$ is superspecial. We have natural homomorphisms

$$
\alpha: \operatorname{Hom}(E, X) \otimes_{R} X \longrightarrow X \quad \text { and } \beta: N \longrightarrow \operatorname{Hom}\left(E, N \otimes_{R} E\right) \text {. }
$$

In order to understand these functors $\operatorname{Hom}(E,-)$ and $-\otimes_{R} E$, the following two observations are useful.

(i) Given an abelian variety $X$ over $k$, there exists a superspecial abelian variety $S^{0}(X)$ and a homomorphism $\rho: S^{0}(X) \rightarrow X$ with the following universal property: if $Y$ is a superspecial abelian variety, any homomorphism $f: Y \rightarrow X$ factors through $\rho$, see [29], 1.8. Further, $\rho$ is an isogeny if and only if $X$ is supersingular.

(ii) The class number of $Q_{p, \infty}$ equals 1 . This means that for a maximal order $R$ as above, every torsion-free right $R$-module of finite type is free, see $[57]$.

Combining these two remarks, we find that $\operatorname{Hom}(E, X)=$ $\operatorname{Hom}\left(E, S^{0}(X)\right)$, so that $\operatorname{Hom}(E, X) \otimes_{R} X \cong S^{0}(X)$, and $\alpha$ is just the isogeny $\rho$. In the other direction, if $N$ is a right $R$-module of finite type, consider the natural map $\pi: N \rightarrow N^{\prime}:=N / \operatorname{Tors}(N)$. Then $N \otimes_{R} E \stackrel{\sim}{\rightarrow} N^{\prime} \otimes_{R} E$. We have $\operatorname{Hom}\left(E, N \otimes_{R} E\right) \cong N^{\prime}$, and via this isomorphism $\beta$ is just the map $\pi$.

(7.14) Lemma. Choose notation as in (7.12); in particular $X$ is superspecial. There is a natural bijection

$$
\left\{\begin{array}{c}
\text { symmetric isomorphisms } \\
X \stackrel{\sim}{\rightarrow} X^{t}
\end{array}\right\} \stackrel{\sim}{\rightarrow}\left\{\begin{array}{c}
\text { perfect hermitian } \\
\text { forms on } M
\end{array}\right\}
$$

which restricts to a bijection

$$
\left\{\begin{array}{c}
\text { principal polarizations } \\
X \underset{\sim}{\rightarrow} X^{t}
\end{array}\right\} \stackrel{\sim}{\rightarrow}\left\{\begin{array}{l}
\text { positive definite perfect } \\
\text { hermitian forms on } M
\end{array}\right\}
$$


Proof. Given a homomorphism $\mu: X \rightarrow X^{t}$, let $\psi=\psi_{\mu}$ be the hermitian form on $M$ given by

$$
\psi\left(h, h^{\prime}\right)=\left(E \stackrel{h^{\prime}}{\longrightarrow} X \stackrel{\mu}{\longrightarrow} X^{t} \stackrel{h^{t}}{\longrightarrow} E^{t} \stackrel{\lambda^{-1}}{\longrightarrow} E\right) .
$$

In the opposite direction, given a hermitian form $\psi$ on $M$, consider the associated homomorphism $\tilde{\psi}: M \rightarrow M^{\dagger}$, and define $\mu=\mu_{\psi}: X \rightarrow X^{t}$ by

$$
\mu_{\psi}=\tilde{\psi} \otimes \lambda: X=M \otimes_{R} E \longrightarrow X^{t}=M^{\dagger} \otimes_{R} E^{\dagger} .
$$

One checks that $\mu \mapsto \psi_{\mu}$ and $\psi \mapsto \mu_{\psi}$ are inverse to each other, and that a perfect form $\psi$ correspond to an isomorphism $\mu$. This gives the first bijection. Under this bijection the principal polarizations correspond to the positive definite perfect hermitian forms: this follows from what is explained in [37], §21, Application III.

(7.15) We keep the notations introduced in (7.12). Let $\ell$ be a prime number different from $p$. Write $R_{\ell}:=R \otimes_{\mathbb{Q}} \mathbb{Z}_{\ell}$; then there exists an isomorphism $R_{\ell} \cong$ $M_{2}\left(\mathbb{Z}_{\ell}\right)$.

The canonical pairing

$$
e_{\ell}: T_{\ell} E \times T_{\ell} E^{\dagger} \longrightarrow \mathbb{Z}_{\ell}(1)
$$

has the property that $e_{\ell}\left(r \xi, \xi^{\prime}\right)=e_{\ell}\left(\xi, r^{\dagger} \xi^{\prime}\right)$ for all $r \in R, \xi \in T_{\ell} E$ and $\xi^{\prime} \in$ $T_{\ell} E^{\dagger}$. Using this, one can show that there exists a unique bi-additive form

$$
\tilde{e}_{E}: T_{\ell} E \times T_{\ell} E^{\dagger} \longrightarrow R_{\ell}(1)
$$

such that

$$
\begin{aligned}
& e_{E}\left(\xi, \xi^{\prime}\right)=\operatorname{Trd}\left(\tilde{e}_{E}\left(\xi, \xi^{\prime}\right)\right), \quad \text { and } \\
& \tilde{e}_{E}\left(r_{1} \xi, r_{2} \xi^{\prime}\right)=r_{1} \cdot \tilde{e}_{E}\left(\xi, \xi^{\prime}\right) \cdot r_{2}^{\dagger}
\end{aligned}
$$

for all $\xi \in T_{\ell} E$ and $\xi^{\prime} \in T_{\ell} E^{\dagger}$, and $r_{1}, r_{2} \in R$.

Next we remark that the canonical "evaluation" pairing ev: $M \times M^{\dagger} \longrightarrow R$ has the property that

$$
\mathrm{ev}\left(m r_{1}, m^{\prime} r_{2}\right)=r_{2}^{\dagger} \cdot \mathrm{ev}\left(m, m^{\prime}\right) \cdot r_{1} .
$$

We have canonical isomorphisms $T_{\ell} X \cong M \otimes_{R} T_{\ell} E$ and $T_{\ell} X^{t} \cong M^{\dagger} \otimes_{R}$ $T_{\ell} E^{\dagger}$. Taking these as identifications, the canonical pairing $e_{X}: T_{\ell} X \times T_{\ell} X^{\dagger} \longrightarrow$ $\mathbb{Z}_{\ell}(1)$ is given by

$$
e_{X}\left(m \otimes \xi, m^{\prime} \otimes \xi^{\prime}\right)=\operatorname{Trd}\left(\operatorname{ev}\left(m, m^{\prime}\right) \cdot \tilde{e}_{E}\left(\xi, \xi^{\prime}\right)\right) .
$$


Let us note here that the form

$$
\left((m, \xi),\left(m^{\prime}, \xi^{\prime}\right)\right) \mapsto \operatorname{ev}\left(m, m^{\prime}\right) \cdot \tilde{e}_{E}\left(\xi, \xi^{\prime}\right)
$$

does not descend to a well-defined form on $T_{\ell} X \times T_{\ell} X^{t}$. However, using (1-3) together with the fact that $\operatorname{Trd}$ is a trace form $($ so $\operatorname{Trd}(\alpha \beta)=\operatorname{Trd}(\beta \alpha))$ we find that the right hand side of (4) is well-defined on $T_{\ell} X \times T_{\ell} X^{t}$.

(7.16) Let $a$ be a positive integer, and let $n:=\ell^{a}$. Recall that we write $H_{n}$ for the standard hermitian pairing on $(R / n R)^{g}$. Assume given a primitive $n$-th root of unity in $k$, via which we identify $\mathbb{Z} / n \mathbb{Z}$ and $\mathbb{Z} / n \mathbb{Z}(1)=\mu_{n}(k)$. Using what was explained in (7.15), we find that the Weil pairing $e_{n}^{\lambda}: E[n] \times E[n] \longrightarrow \mathbb{Z} / n \mathbb{Z}$ lifts to a pairing

$$
\tilde{e}_{n}^{\lambda}: E[n] \times E[n] \longrightarrow R / n R
$$

Consider the pairing

$$
\theta:\left((R / n R)^{g} \otimes_{R} E[n]\right) \times\left((R / n R)^{g} \otimes_{R} E[n]\right) \longrightarrow \mathbb{Z} / n \mathbb{Z}
$$

given by $\theta\left((x \otimes P),\left(x^{\prime} \otimes P^{\prime}\right)\right)=\operatorname{Trd}\left(H_{n}\left(x, x^{\prime}\right) \cdot \tilde{e}_{n}^{\lambda}\left(P, P^{\prime}\right)\right)$. One checks that this is a well-defined symplectic pairing. In other words, if $\Phi_{n}$ is the standard symplectic pairing on $(\mathbb{Z} / n \mathbb{Z})^{2 g}$ then there exists an isometry

$$
\left((R / n R)^{g} \otimes_{R} E[n], \theta\right) \stackrel{\sim}{\rightarrow}\left((\mathbb{Z} / n \mathbb{Z})^{2 g}, \Phi_{n}\right) .
$$

More generally, if $n$ is an arbitrary positive integer prime to $p$ then we find that there exist isometries as above by writing $n=\ell_{1}^{a_{1}} \ldots \ell_{r}^{a_{r}}$ and applying the preceding to each of the factors $\ell_{i}^{a_{i}}$.

(7.17) Lemma Let $n$ be a positive integer, not divisible by $p$. Assume chosen a primitive $n$-th root of unity in $k$. Fix an isometry as in (7.16). Let $\mu$ be a principal polarization of $X$ which, as in (7.14), corresponds to a perfect hermitian form $\psi$ on $M$. Then there is a natural bijection

$$
\left\{\begin{array}{c}
\text { symplectic level- } n \\
\text { structures on }(X, \lambda)
\end{array}\right\} \quad \sim\left\{\begin{array}{c}
\text { level- } n \text {-markings } \\
\text { of }(M, \psi)
\end{array}\right\}
$$

Proof. For simplicity, let us assume that $n$ is a power of a prime number $\ell$. (The general case is easily reduced to this.) Suppose given a level- $n$-marking of $(M, \psi)$. Using what was explained in $(7.15)$ we find that the induced isomorphism

$$
X[n]=(M / n M) \otimes_{R} E[n] \stackrel{\sim}{\rightarrow}(R / n R)^{g} \otimes_{R} E[n]
$$

gives an isometry

$$
\left(X[n], e_{n}^{\mu}\right) \stackrel{\sim}{\rightarrow}\left((R / n R)^{g} \otimes_{R} E[n], \theta\right),
$$


where $\theta$ is as in (7.16). Composing with the fixed isometry (7.16) this gives a symplectic level- $n$-structure of $(X, \mu)$. This defines the map in (7.17).

By an easy "integral" variant of (7.6) we see that Aut $\left((R / n R)^{g}, H_{n}\right) \cong$ $\mathrm{Sp}_{2 g}(\mathbb{Z} / n \mathbb{Z})$. In particular, both sides of $(7.17)(1)$ are principal homogeneous under $\operatorname{Sp}_{2 g}(\mathbb{Z} / n \mathbb{Z})$. Further, one easily sees that the map (7.17)(1) just defined is compatible with these structures of homogeneous spaces. Hence our map is a bijection.

(7.18) Proof of Proposition (7.3). Let $x$ and $x^{\prime}$ be points of $\Sigma(k)$, corresponding to triples $(X, \mu, \eta)$ and $\left(X^{\prime}, \mu^{\prime}, \eta^{\prime}\right)$, respectively. By Lemma (7.9) we can embed the corresponding $R$-modules $M:=\operatorname{Hom}(E, X)$ and $M^{\prime}:=$ $\operatorname{Hom}\left(E, X^{\prime}\right)$ as perfect hermitian $R$-lattices in $(V, \Psi)$ in such a way that $M \cap M^{\prime}$ has $p$-power index in both $M$ and $M^{\prime}$ and that the given level- $n$-markings of $M$ and $M^{\prime}$ agree. As $\mathfrak{p} \subset R$ is a principal ideal, see (ii) of Remark (7.13), we can apply Lemma (7.11). This gives us perfect hermitian lattices

$$
M=L_{0}, L_{1}, \ldots, L_{m}=M^{\prime}
$$

with $L_{j} /\left(L_{j} \cap L_{j+1}\right) \cong k(\mathfrak{p}) \cong L_{j+1} /\left(L_{j} \cap L_{j+1}\right)$. Fix $j \in\{0, \ldots, m-1\}$. Write $N:=L_{j} \cap L_{j+1}$, and consider the homomorphisms

$$
X_{j}:=L_{j} \otimes_{R} E \quad \stackrel{f}{\longleftarrow} \quad Y:=N \otimes_{R} E \quad \stackrel{h}{\longrightarrow} \quad X_{j+1}=L_{j+1} \otimes_{R} E
$$

induced by the inclusions $N \hookrightarrow L_{j}$ and $N \hookrightarrow L_{j+1}$, respectively. Via the correspondence (7.14), we have principal polarizations $\mu_{j}$ on $X_{j}$ and $\mu_{j+1}$ on $X_{j+1}$; these satisfy $f^{*} \mu_{j}=h^{*} \mu_{j+1}$. The given level-n-markings of $M$ and $M^{\prime}$ induce level- $n$-markings on each of the lattices $L_{j}$; we write $\eta_{j}$ for the corresponding symplectic level- $n$-structure of $\left(X_{j}, \mu_{j}\right)$.

We are done if we show that $f$ is a covering satisfying conditions (i)-(iii) of (7.1). Indeed, by symmetry the same then holds for $h$, and we conclude that $\left(X_{j}, \mu_{j}, \eta_{j}\right)$ and $\left(X_{j+1}, \mu_{j+1}, \eta_{j+1}\right)$ lie on the same irreducible component of the locus $L$, as constructed in (7.1).

By construction, $f$ is an isogeny of superspecial abelian varieties, and $\operatorname{deg}(f)$ is a $p$-power. (It is easily seen that $f$ induces an isomorphism $T_{\ell} Y \stackrel{\sim}{\rightarrow} T_{\ell} X_{j}$ for all primes $\ell \neq p$.) Further, $f$ is minimal, in the sense that it does not admit a non-trivial factorization. Hence $\operatorname{Ker}(f) \cong \alpha_{p}$. This implies that $\operatorname{Ker}\left(f^{*} \mu_{j}\right)$ is either isomorphic to $\alpha_{p} \times \alpha_{p}$, or it is a non-trivial extension of $\alpha_{p}$ by $\alpha_{p}$. But in the latter case $\operatorname{Ker}\left(f^{*} \mu\right)$ has a unique subgroup scheme of rank $p$, so that necessarily $\operatorname{Ker}(f)=\operatorname{Ker}(h)$. This is in contradiction with our construction. 


\section{Abelian varieties with $a=g-1$}

In this section we work over an algebraically closed field $k \supset \mathbb{E}_{p}$. We consider principally polarized abelian varieties and principally quasi-polarized $p$-divisible groups. We study strata defined by an elementary sequence $\varphi$ with $\varphi(g)=1$. These describe abelian varieties of dimension $g$ with $a(\varphi)=a(X)=g-1$. Results of this section are rather elementary once you know the DieudonnéManin classification; we do not use results of the previous sections. We use a result in [29], and we use some notations as explained in [19]. For the notation $G_{m, n}$ we refer to $(15.5)$.

(8.1) For every pair $m, n \in \mathbb{Z}_{>0}$ of coprime integers there exists a $p$-divisible group $H_{m, n}$, defined over $\mathbb{E}_{p}$, isogenous with $G_{m, n}$, which is characterized by the fact that its endomorphism ring over an algebraically closed field is maximal within the isogeny class; see [19], Section 5. This $p$-divisible group can be defined by the Dieudonné module given by the semimodule $[0, \infty)$, in the notation as explained in [19]. If $m>1$ and $n>1$ there exist $p$-divisible groups isogenous with $G_{m, n}$ not isomorphic with $H_{m, n}$.

We know that $a\left(H_{m, n}\right)=\min (m, n)$ (as is easy to see form the defining semi-module. However this property is in general not sufficient to characterize $H_{m, n}$ within its isogeny class, but in the cases used in this section it will be the case (see the lemma below). One can feel the object $H_{m, n}$ as the (unique) "minimal" one in the isogeny class of $G_{m, n}$, and groups with $a(G)<1$ as the most general ones; this can be made precise, see [19], Section 5, especially see (5.7).

(8.2) If $G$ is a formal group isogenous with $G_{m, n}$ then

$$
a(G) \leq m, \quad a(G) \leq n
$$

Moreover we know that the maximum $a(G)=\min (m, n)$ is achieved within this isogeny class.

See [42], page II.15-10, see [50].

A proof is not difficult; e.g. using notation as introduced in [19] we see: let $G \sim G_{m, n}$ over an algebraically closed field, and let $A=\operatorname{Type}(G)$ be the semi-module associated with $G$. Consider

$$
A \backslash(m+A \cup n+A) \rightarrow \mathbb{Z} \rightarrow \mathbb{Z} / m
$$

This map is injective. Hence

$$
\#(A \backslash(m+A \cup n+A))=a(A)=a(G) \leq m .
$$

The same holds for the natural mapping to $\mathbb{Z} / n$. This proves the desired inequality. As moreover $a\left(H_{m, n}\right)=\min (m, n)$ we are done. 
Remark. If the isogeny type of a $p$-divisible group $G$ is given, consider "an isogeny factor", i.e. the image of some $G_{m, n}$ appearing in the isogeny type under an isogeny into $G$. In general these "isogeny factors" are not direct summands, and it is usually difficult to decide which member of the isogeny class of $G_{m, n}$ appears in this way. However in the situation studied in this section all these questions turn out to have a unique and simple answer.

In this paper we use $V-U$ to denote the complement of $U$ in $V$; however in the proof above we use $V \backslash U$ for this notion, in order to avoid confusion with subtraction.

(8.3) Theorem. Let $X$ be an abelian variety of dimension $g$ over an algebraically closed field of characteristic $p$ which admits a principal polarization. Suppose $\operatorname{ES}(X)=\varphi$ with $\varphi(g)=1$, i.e. $a(X)=g-1$. Then:

(I) either there exists an integer $b$ with $0 \leq b \leq\left[\frac{q-1}{2}\right]$ and an isomorphism

$$
X\left[p^{\infty}\right] \cong H_{b+1, b} \bigoplus(g-2 b-1) \cdot G_{1,1} \bigoplus H_{b, b+1}
$$

and in this case

$$
\varphi=(0, \cdots, 0, \overbrace{1, \cdots, 1}^{g-b}), \quad|\varphi|=g-b,
$$

(II) or there exists an integer $r$ with $0<r \leq\left[\frac{g}{2}\right]$, there exists an isogeny $X \sim$ $E^{g}$, where $E$ is a supersingular elliptic curve (i.e. $X$ is supersingular), and

$$
\varphi=(0, \cdots, 0, \overbrace{1, \cdots, 1}^{r}), \quad|\varphi|=r
$$

(and the structure of $X\left[p^{\infty}\right]$ will be explained in the proof). Note that for all $b$ as in (I) and all $r$ as in (II) we have $g-b>r$ and $0 \leq r \leq\left[\frac{g-1}{2}\right] \leq g-b$; the value of $|\varphi|$ determines in which case we are.

As a corollary of this theorem and its proof we deduce:

$$
\text { Corollary. Let } X \text { be an abelian variety with }
$$

$$
\operatorname{ES}(X)=\varphi:=(0, \cdots, 0,1)
$$

Then $X$ is as described in the construction (7.1). In particular

$$
L^{o}=S_{(0, \cdots, 0,1)}, \quad L=S_{(0, \cdots, 0,1)} \cup \Sigma=\left(S_{(0, \cdots, 0,1)}\right)^{c} .
$$

From the theorem we conclude that $S_{(0, \cdots, 0,1)} \subset L$; hence the corollary follows from (7.2) and the previous theorem.

$\square(8.3) \Rightarrow(8.4)$ 
The proof of this theorem will be given in various steps. In the first step we analyze the isogeny type of $X\left[p^{\infty}\right]$.

First step. Suppose $a(X)=g-1$. From (8.2) it follow that we are in one of the following cases:

(I.0) either $f(X)=1$; we claim: in this case

$$
X\left[p^{\infty}\right] \cong G_{1,0} \oplus(g-1) \cdot G_{1,1} \oplus G_{0,1},
$$

and we conclude $\varphi(1)=1$; hence $\varphi=(1, \cdots, 1)$; this is case I.0;

(I.b) or $f(X)=0$ and there exists an integer $b$ with $0<b \leq\left[\frac{g-1}{2}\right]$ and an isogeny

$$
X\left[p^{\infty}\right] \sim G_{b+1, b} \bigoplus(2 g-2 b-1) \cdot G_{1,1} \bigoplus G_{b, b+1}
$$

we shall analyze this case;

(II) or

$$
X\left[p^{\infty}\right] \sim g \cdot G_{1,1} ;
$$

we shall describe all supersingular cases with $a(X)=g-1$.

Indeed, the fact that $a(X)=g-1$ limits the possibilities of the formal isogeny types to the cases as indicated. If $f(X)=1$ and $a(X)=g-1$ we see (note that the base field is algebraically closed) that $X\left[p^{\infty}\right] \cong G_{1,0} \oplus G_{0,1} \oplus G$ with $a(G)=\operatorname{dim}(G)$. This proves the first statement. The remaining possibilities have been listed.

(8.5) Lemma. Let $b \in \mathbb{Z}_{>0}$, let $G$ be a $p$-divisible group over an algebraically closed field with $G \sim G_{b+1, b}$, and $a(G)=b$; then $G \cong H_{b+1, b}$.

Proof. The case $b=0$ is well-known; we suppose $b>0$. In [19] we have seen that to every $G \sim G_{b+1, b}$ we can attach a semi-module $A=\operatorname{Type}(G) \subset \mathbb{Z}$. This is a subset of $\mathbb{Z}$ bounded from below, and stable under $+m=+(b+1)$ and $+n=+b$; let $t \in A$ be the smallest element.

We show that (under all conditions mentioned) from $a(A)=b$ it follows that $A=[t, \infty)$; in fact, let $C=A \backslash((b+A) \cup((b+1)+A))$, hence $\#(C)=a(A)$; if there exist $x, y \in \mathbb{Z}$ such that $t+x+1, \cdots, t+y-1 \notin A$, then

$$
\#\left(\left(\cup_{j}[t+x+1+j \cdot(b+1), t+y+b-1+j \cdot b]\right) \cap C\right)<y-x-1 ;
$$

we see that such gaps in $[t, t+B-1] \cap A$ do not appear if $a(A)=b$; we conclude that $A=[t, \infty)$. This implies $G \cong H_{b+1, b}$, which proves the lemma.

(8.6) Remark. Let $m, n \in \mathbb{Z}$ with $m>n+1>n>1$. The semi-module $A=\{0\} \cup[2, \infty]$ has $a(A)=n$. We see that in this case there exists $G \cong G_{m, n}$ with $a(G)=n=\min (m, n)$ and $G \not H_{m, n}$. 
(8.7) Second step. Lemma. Let $G$ be a p-divisible group over an algebraically closed field, which admits a principal polarization, such that $G \sim$ $H_{b+1, b} \oplus(g-2 b-1) \cdot G_{1,1} \oplus H_{b, b+1}$ and $a(G)=g-1$. Then

$$
G \cong H_{b+1, b} \bigoplus(2 g-2 b-1) \cdot G_{1,1} \bigoplus H_{b, b+1}
$$

and we have

$$
\operatorname{ES}(G)=\varphi=(0, \cdots, 0,1, \cdots 1), \quad|\varphi|=g-b .
$$

Proof. Let $I, S, J \subset G$ be the images of the three factors under an isogeny $H_{b+1, b} \oplus(2 g-2 b-1) \cdot G_{1,1} \oplus H_{b, b+1} \rightarrow G$. We are going to use that in the isogeny classes of $I, S, J$ there is a unique (up to isomorphism) group with maximal $a$-number. We claim: $a(I)=b=a(J)$ and $a(S)=2 g-2 b-1$ and hence $I \cong H_{b+1, b}$, and $S \cong(2 g-2 b-1) \cdot G_{1,1}$, and $J \cong H_{b, b+1}$; for a $p$ divisible group $Y$ over a perfect field we write $\alpha(Y) \subset Y$ for the smallest subgroup scheme containing all $\alpha_{p} \subset Y$ (denoted by $A(Y)$ in [29], 1.5, but we have used that notation already for a different purpose). The exact sequence $\alpha(I) \subset Y \rightarrow Y /(\alpha(I))$ together with the fact that $a(Y)$ and $a(Y /(\alpha(I)))$ are "maximal" show that $a(I)=b$; analogous statements for $S$ and for $J$; these imply the existence of the isomorphisms indicated, hence the claim. Next we show that the natural map $I \oplus S \oplus J \rightarrow I+S+J=G$ is an isomorphism. We show this for $S \oplus J \rightarrow S+J$; because the a-numbers are "maximal" we see that

$$
0 \rightarrow \alpha(S) \rightarrow \alpha(S+J) \rightarrow \alpha((S+J) / S) \rightarrow 0
$$

is exact; it follows that the

$$
\operatorname{Ker}(S \oplus J \rightarrow S+J) \cap \alpha(S \oplus J)=0,
$$

which proves $S \oplus J \cong S+J$. An analogous statement holds for $I+S$ and for $I+J$. From these the result follows.

(8.8) Remark. We see cases where a $p$-divisible group $G$ is determined up to isomorphism by $G[p]$ : the case $f(G)=\operatorname{dim}(G)$, the case $a(G)=\operatorname{dim}(G)$, and the case as in (I) of the theorem. However in general $G[p]$ does not determine $G$. We have a general conjecture which illustrates what should be (im) possible, see (14.7), and (14.8).

(8.9) Third step. Suppose we are in the situation as in (II), i.e. $X$ is a supersingular abelian variety with a principal polarization with $a(X)=g-1$. We use the description of the locus $\mathcal{S}_{g-1}(a \geq g-1)$ as given in [29], Section 9 , in particular in (9.10) and (9.11). From that description we see that in this 
case there exists an integer $r$ with $0<r \leq\left[\frac{g}{2}\right]$, an isomorphism $\xi_{p}(X) \cong$ $(g-2 r) \cdot G_{1,1} \oplus G$, and an exact sequence

$$
0 \rightarrow\left(\alpha_{p}\right)^{r} \rightarrow(2 r) \cdot G_{1,1} \rightarrow G \rightarrow 0,
$$

with $a(G)=2 r-1$. We assume the integer $r$ is chosen to be the minimal one allowing this structure. The polarization in that case gives a form on the Dieudonné-module, with $\left\langle e_{i}, \mathcal{F}\left(e_{i}\right)>\right.$ non-zero for $\left.1 \leq i<2 g-2 r\right\rangle$ and $\left\langle e_{2 g-2 j}, e_{2 g-2 j+1}>\right.$ non-zero for $r \geq j \geq 1$. An explicit computation shows that in this case $\varphi=(0, \cdots, 0,1, \cdots 1), \quad|\varphi|=r$. This ends the proof of the theorem.

\section{$9 \quad$ Standard types}

In this section we attach to every elementary sequence $\varphi$ a finite group scheme $N_{\varphi}$ plus a non-degenerate alternating pairing $\langle\rangle=,\left\langle,>_{\text {st }}\right.$ on $A_{\varphi}:=\mathbb{D}\left(N_{\varphi}\right)$ such that the pair $\left(N_{\varphi},<,>\right)$ is defined over $\mathbb{E}_{p}$, and such that $\operatorname{ES}\left(N_{\varphi}\right)=\varphi$.

Then we show that a "polarized $\mathrm{BT}_{1}$ truncated group scheme" over an algebraically closed field $k$ is isomorphic (over $k$ ) with the standard type given by its elementary sequence. This result will be used in (12.5), where we use that two polarized $\mathrm{BT}_{1}$ group schemes over an algebraically closed field with equal elementary sequences are isomorphic.

Remark. It might seem strange that we define $\langle$,$\rangle on the Dieudonné module$ (and not on the group scheme itself). If we would avoid characteristic 2 , in fact we could work with anti-symmetric morphisms on group schemes, prove the results we want to achieve, and develop a mechanism which works over any base in characteristic $p \neq 2$. However in characteristic $=2$ we do not see how this simplification can be carried out with success. We have chosen for a unified treatment applicable in all characteristics. For a further discussion we refer to Section 12.

I do not know how to characterize the fact that $\langle\rangle:, A \times A \rightarrow K$ is alternating on $A:=\mathbb{D}(N)$ in terms of the related symmetric $\zeta: N \rightarrow N^{D}$ in chase the characteristic of the perfect base field $K$ equals $p=2$.

Reminder: $\mathbb{D}\left(V: N^{(p)} \rightarrow N\right)=\left(\mathcal{F}: A^{(p)} \rightarrow A\right)$. The constructions involving $V$ and $F^{-1}$ on $N$ we find back as construction involving $\mathcal{F}$ and $\mathcal{V}^{-1}$ on $A=\mathbb{D}(N)$.

Remark. We use in this paper the following notation, also see Section 12: let $(X, \lambda)$ be a principally polarized abelian variety over a perfect field $K$ of characteristic $p$; write $N:=X[p]$, with Dieudonné module $A=\mathbb{D}(N)$; the polarization induces a symmetry on $N$, and it defines a pairing $<,>: A \times A \rightarrow$ $K$. We write $(N,<,>)=:(X, \lambda)[p]$ for this "polarized $\mathrm{BT}_{1}$ truncated group scheme". 
Remark. In what follows we use a classification of a $\mathrm{BT}_{1}$ over an algebraically closed field found by Kraft, see [27], and rediscovered independently much later by the present author. That structure is not very difficult to describe. However the classification of "polarized $\mathrm{BT}_{1}$ truncated group schemes" turns out to be more involved, as will be seen below.

(9.1) Construction. Suppose given an elementary sequence

$$
\{\varphi(1), \cdots, \varphi(g)\}=\varphi \in \Phi, \quad \varphi(0)=0,
$$

and let $\psi$ be the related final sequence:

$$
\psi(i)=\varphi(i) \quad \text { for } \quad 0 \leq i \leq g, \quad \text { and } \quad \psi(g-i)=\varphi(i)+g-i \quad \text { for } \quad 0 \leq i \leq g .
$$

Consider the set of indices where the $\psi$-sequence jumps:

$$
1 \leq m_{1}<m_{2}<\cdots<m_{g} \leq 2 g
$$

this is the set of all integer $i$ such that $\psi(i-1)<\psi(i)$. We denote by

$$
1 \leq n_{g}<n_{g-1}<\cdots<n_{1} \leq 2 g
$$

the complimentary set, i.e. the set of indices where the $\psi$-sequence does not jump. Note that:

$$
m_{i}+n_{i}=2 g+1 \text { for } 0 \leq i \leq g .
$$

For convenience we write $m_{0}=0,2 g+1=m_{g+1}, \quad n_{g+1}=0, \quad n_{0}=2 g+1$.

Given $\varphi \in \Phi$ we are going to construct a covariant Dieudonné-module $A_{\varphi}$ with a non-degenerate alternating pairing over $\mathbb{F}_{p}$. The related group scheme with pairing will be a polarized $\mathrm{BT}_{1}$, which will be denoted by $\left(N_{\varphi},<,>\right)$. It will be called the standard type given by $\varphi$.

We construct $A=A_{\varphi}$ as the vector space of dimension $2 g$ over $\mathbb{F}_{p}$ generated by a base $\left\{Z_{1}, \cdots, Z_{2 g}\right\}$. We write

$$
Z_{m_{i}}=X_{i} \quad \text { and } \quad Z_{n_{i}}=Y_{i} \quad \text { for } \quad 1 \leq i \leq g
$$

note that the numbering of the $Y_{i}$ is decreasing, and the numbering of the $X_{i}$ is increasing in $\left\{Z_{j} \mid j\right\}$. We define

$$
\mathcal{F}: A \rightarrow A \quad \text { by } \quad \mathcal{F}\left(X_{i}\right):=Z_{i}, \quad \mathcal{F}\left(Y_{i}\right)=0 \quad \text { for } \quad 0 \leq i \leq g .
$$

We define the alternating pairing by

$$
<X_{i}, Y_{j}>=\delta_{i, j}, \quad<X_{i}, X_{j}>=0, \quad<Y_{i}, Y_{j}>=0 \text { for } 0 \leq i, j \leq g .
$$

Note that these determine the action of $\mathcal{V}$; in fact $\mathcal{V}: M \rightarrow M$ is given by

$$
\mathcal{V}\left(Z_{i}\right)=0, \quad \mathcal{V}\left(Z_{2 g-i+1}\right)= \pm Y_{i} \quad \text { for } \quad 0 \leq i \leq g
$$


where $\mathcal{V}\left(Z_{2 g-i+1}\right)=+Y_{i}$ if $Z_{2 g-i+1} \in\left\{Y_{g}, \cdots, Y_{1}\right\}$ and $\mathcal{V}\left(Z_{2 g-i+1}\right)=-Y_{i}$ if $Z_{2 g-i+1} \in\left\{X_{1}, \cdots, X_{g}\right\}$.

It follows that $\mathcal{F}, \mathcal{V}$ and $\langle$,$\rangle are connected by the rules they should$ satisfy:

$$
\langle\mathcal{F}(a), b\rangle=\langle a, \mathcal{V}(b)\rangle \quad \text { for all } a, b \in A
$$

note that the base field is $\mathbb{F}_{p}$; hence the Dieudonné modules are over the ring $\mathbb{F}_{p}[\mathcal{F}, \mathcal{V}]$, which is commutative in this case. Note that

$$
\operatorname{Im}(\mathcal{F})=\mathbb{E}_{p} \cdot Z_{1} \oplus \cdots \oplus \mathbb{F}_{p} \cdot Z_{g}=\operatorname{Ker}(\mathcal{V})
$$

and

$$
\operatorname{Ker}(\mathcal{F})=\mathbb{F}_{p} \cdot Y_{g} \oplus \cdots \oplus \mathbb{F}_{p} \cdot Y_{1}=\operatorname{Im}(\mathcal{V}) .
$$

We denote by $\left(N_{\varphi},<,>_{\text {st }}\right)$ the associated group scheme with this nondegenerate pairing on $A_{\varphi}=\mathbb{D}\left(N_{a}\right)=\oplus \mathbb{F}_{p} \cdot Z_{i}$, all defined over $\mathbb{E}_{p}$. From the formulas just derived we see that this is a "polarized $\mathrm{BT}_{1}$ of rank $p^{2 g}$ defined over $\mathbb{F}_{p} "$, see below. Moreover we see that the submodules $A_{i}=\oplus_{j=1}^{j=i} \mathbb{F}_{p} \cdot Z_{j}$ define a final filtration on $N=N_{\varphi}$, and we see that the final type of this final filtration equals $\varphi$.

(9.2) Definition. Let $K$ be a perfect field. We say that $(N,<>)$ is a polarized $\mathrm{BT}_{1}$ truncated group scheme, or: a polarized $\mathrm{BT}_{1}$, defined over $K$, if $N$ is a $\mathrm{BT}_{1}$ over $K$, with Dieudonné module $A:=\mathbb{D}(N)$ and $\langle,>: A \times A \rightarrow K$ is an alternating, non-degenerate pairing such that $\langle\mathcal{F} x, y\rangle=\langle x, \mathcal{V} y\rangle^{p}$ for all $x, y \in A$.

(9.3) Conclusion. For every elementary sequence $\varphi \in \Phi$ we have constructed a polarized $B T_{1}\left(N_{\varphi},<,>\right)$ defined over $\mathbb{E}_{p}$ with (covariant) Dieudonné module $\left(A_{\varphi},<,>\right)$, with a final filtration given by a symplectic $\mathbb{F}_{p}$-base $\left\{Y_{g}, \cdots, Y_{1}, X_{1}, \cdots, X_{g}\right\}$ such that $Y_{i} \in \operatorname{Ker}(\mathcal{F})$ for every $i$ and such that every base element under $\mathcal{F}$ is either mapped to zero or to a base vector, and such that $\operatorname{ES}\left(N_{\varphi}\right)=\varphi$. It will be called the standard type defined by $\varphi$. The base $\left\{Z_{1}, \cdots, Z_{2 g}\right\}=\left\{Y_{g}, \cdots, Y_{1}, X_{1}, \cdots, X_{g}\right\}$ will be called a standard base for $A_{\varphi}$. In case confusion is possible, we will write $\left.<,\right\rangle_{\text {st }}$ for the "standard pairing" on the "standard module" $A_{\varphi}$. A final filtration is given by $A_{i}=\oplus_{j=1}^{j=i} \mathbb{E}_{p} \cdot Z_{j}$.

(9.4) Theorem. Suppose $k$ is an algebraically closed field of characteristic $p$. Let $(N,[]$,$) be a polarized B T_{1}$ of rank $p^{2 g}$ defined over $k$. Suppose $\varphi=\operatorname{ES}(N)$ is the elementary sequence determined by $N$. Then

$$
(N,[,]) \cong\left(N_{\varphi},<,>_{\mathrm{st}}\right) \otimes_{\mathbb{F}_{p}} k
$$

Remark. This isomorphism is not unique in general. 
As Ben Moonen shows, see [33], a different, easier proof of this theorem can be given in case the characteristic of the base field is not equal to two. We could not generalize that proof to include the case $\operatorname{char}(k)=p=2$. Below I give a proof which covers all cases uniformly. It seems desirable to simplify the methods of this section.

Strategy of the proof. We study on the one hand all pairings on $A=\mathbb{D}(N)$ such that a given $(\mathcal{F}, \mathcal{V})$-base is in standard form on the associated grade module; on the other hand we study all transformations changing a $(\mathcal{F}, \mathcal{V})$ base into another such base. We show that the group of these transformations acts transitively on the set of all such pairings, see (9.20). In order to carry out this programme we study the combinatorics related with the values of a pairing on base vectors, and the combinatorics related to the coefficients needed in such transformations.

The proof of the theorem will be given in several steps.

(9.5) Let $(X, \lambda)$ be a principally polarized abelian variety. Then $N:=X[p]$ is a symmetric $\mathrm{BT}_{1}$. In fact, more is true. The pairing on $X\left[p^{\infty}\right]$ is symmetric, and this implies that the map $\zeta: X[p]=: N \rightarrow N^{D}$ is anti-symmetric, see Section 12 for more details. However more is true: if $(X, \lambda)$ is an abelian variety over a perfect field $K \supset \mathbb{E}_{p}$, the symmetry $\zeta: N \rightarrow N^{D}$ obtained defines a pairing $<,>: A \times A \rightarrow K$ on $A=: \mathbb{D}(N)$ which is alternating.

Indeed, the pairing on $\mathbb{D}\left(X\left[p^{\infty}\right]\right.$ with values in the characteristic zero domain $W_{\infty}(K)$ is symmetric, and the result follows (for details, see (12.2)).

(9.6) Proposition. Let $k$ be an algebraically closed field. Let $(N,<,>)$ be a polarized $B T_{1}$ truncated group scheme defined over $k$, and let $\zeta: N \rightarrow N^{D}$ be the symmetry defined by $\langle$,$\rangle . The canonical filtration on N$ can be refined to a final filtration of $(N, \zeta)$.

Remark. For a given $(N,<,>)$ the number of final filtrations on $(N,<,>)$ is finite. We will give a reformulation of this proposition (in a special case) in (9.13).

Proof. We consider steps in the canonical filtration of $A:=\mathbb{D}(N)$. In $(5.10)$ we have constructed $(\mathcal{F}, \perp)$-cycles in the set of partial quotients in the Dieudonné module of the canonical filtration; such a cycle consists of vertices, each is a quotient in the canonical filtration of the Dieudonné module, edges of the cycle are given by bijective maps $\mathcal{F}$ or $\perp$, and if $\mathcal{F}$ and $\mathcal{F}$ are consecutive edges, there is a "loose edge" $\perp$. We construct a refinement to a final filtration for each of the cycles separately. Let $P$ be one of the $k$-vectorspaces $P=\mathbb{D}\left(N_{j} / N_{j-1}\right)$ as in $(5.10)$. Using a $(\mathcal{F}, \perp)$-cycle in $A$ according to $<,>$, we see that composition of the maps $\mathcal{F}$ and isomorphism $\perp$ give a linear mapping

$$
f:\left(P^{\left(p^{d}\right)}\right)^{\left(D^{\epsilon}\right)} \stackrel{\sim}{\longrightarrow} P
$$


here $d$ is the number of steps involving $\mathcal{F}$, and $\epsilon$ is the parity of the number of steps involving $\perp$. Note that $d>0$. Note that in the same cycle the values $d$ and $\epsilon$ are the same for all edges in a cycle. We distinguish two cases:

(even): suppose the number of edges named $\perp$ in the loop of the cycle is even; in this case the parity of $\epsilon$ is even, i.e.

$$
f:\left(P^{\left(p^{d}\right)}\right) \stackrel{\sim}{\longrightarrow} P
$$

(odd): suppose the number of edges $\perp$ in the loop of the cycle is odd; in this case the parity of $\epsilon$ is odd.

Suppose we are in the case of an (even) cycle. This is case (iii) of Lemma $(5.10)$. We use the "Jordan reduction" for the map $f$ as exposed in [16], or in [6], pp. $232-234$, [56], page 38 . Hence there exists a $k$-base $P=k \cdot e_{1} \oplus \cdots \oplus k \cdot e_{c}$ such that $f\left(e_{i}\right)=e_{i}$. We transport this base through every step of the cycle, pushing is forward by $\mathcal{F}$, or going to the dual base under $\perp$. As $\epsilon$ is even we come back with the same ordered base. This defines in every step of the filtration connected with the cycle a filtration by steps of dimension one; these are related under $\mathcal{F}$ or dual under $\perp$ whenever applicable. This gives a final filtration for all quotients in the cycle studied, hence a final filtration for $N_{\gamma}$, the direct sum of all $N_{j+1} / N_{j}$ for all quotients appearing in this cycle.

Suppose we are in the case of an (odd) cycle. This is case (ii) of Lemma (5.10). Let us consider one odd $(\mathcal{F}, \perp)$-cycle $\gamma$. As we have seen this is constructed from a word $w$ in $\mathcal{F}$ and $\mathcal{V}^{-1}$, describing the combinatorics of the related $\left(\mathcal{F}, \mathcal{V}^{-1}\right)$ cycle on $A$, i.e. the word in $V$ and $F^{-1}$ in the $\left(V, F^{-1}\right)$-cycle on $N$. As $\gamma$ is odd, the word $w$ is self-dual, the number $|w|$ of letters is even; we write $|w|=2 m$. Let $P=P_{0}$ be one of the subquotients of $\mathbb{D}(N)$ appearing in $\gamma$; consider $P_{0}, P_{1}, \cdots, P_{2 m-1}, P_{m}=P$, the subquotients appearing in the $\left(\mathcal{F}, \mathcal{V}^{-1}\right)$-cycle; consider $g_{i}: P=P_{0} \rightarrow P_{i}$, which is obtained by going clockwise $i$ steps through the $\left(\mathcal{F}, \mathcal{V}^{-1}\right)$-cycle given by $w$, with the related maps, using either $\mathcal{F}$ or $\mathcal{V}^{-1}$; it is a $\sigma^{i}$-linear map. We write $g=g_{2 m}: P_{0}=P \rightarrow P=P_{2 m}$. We see that this is a $\sigma^{2 m}$-linear endomorphism of the vector space $P$. Let us write $c:=\operatorname{dim}_{k} P$.

We use the "Jordan reduction" for the map $g$ as exposed in [16], or in [6], pp.232 - 234, [56], page 38. This shows: define $\mathbb{F}:=\mathbb{F}_{p^{2 m}}$; there exists a $\mathbb{F}$-base $\mathcal{P}:=\mathbb{F} \cdot e_{1} \oplus \cdots \oplus \mathbb{F} \cdot e_{c}$, and $g: \mathcal{P} \rightarrow \mathcal{P}$ such that $g\left(e_{i}\right)=e_{i}$, and such that $(\mathcal{P}, g) \otimes_{\mathbb{F}} k \cong(P, g)$. From now on we work with vector spaces over $\mathbb{F}$, in particular we write $g_{i}(\mathcal{P})=\mathcal{P}_{i}$.

We write $\iota:=F^{m}$, i.e. the map $x \mapsto x^{p^{m}}$ when restricted to $\mathbb{F}$ we have $\iota \in \operatorname{Gal}\left(\mathbb{F} / \mathbb{F}_{p^{m}}\right)$. Let us write $g_{m}=: h: P_{0}=P \rightarrow Q=P_{m}$, and analogously $H: \mathcal{P} \rightarrow \mathcal{P}$. We define

$$
\psi: P \times P \rightarrow \mathbb{F} \quad \text { by } \quad \psi(x, y)=<x, h y>.
$$


Remark that $g: P \rightarrow P$ is $\iota$-linear over $k$, hence $g: \mathcal{P} \rightarrow \mathcal{P}$ is linear over $\mathbb{F}$; hence every base change over $\mathbb{F}$ leaves $g$ in diagonal-1-form.

Note that $\psi$ is:

1) non-degenerate,

2) linear in the first variable,

3) $t$-linear in the second variable, and to $\iota "$.

4) $\psi(x, y)=-\psi(y, x)^{\iota}$ for all $x, y \in P$, i.e. " $\psi$ is hermitian with respect

Form this last equality we see that for all $x, y \in \mathcal{P}$ we have $\psi(x, y)=\psi(x, y)^{\iota \cdot \iota}$; this shows that $\psi$ factors as $\psi: \mathcal{P} \rightarrow \mathcal{P}$ :

5) for all $x, y \in \mathcal{P}$ we have $\psi(x, y) \in \mathbb{H}$.

Choose an element $a \in \mathbb{F}^{*}$ such that $b^{L}=-b$, i.e. an element such that $b^{p^{m}-1}=$ -1 . We are going to show that we can choose a $\mathbb{F}$-base $\mathcal{P}=\mathbb{F} \cdot f_{1} \oplus \cdots \oplus \mathbb{F} \cdot f_{c}$ such that on this $\mathbb{F}$-base the form $\psi$ is anti-diagonal with $b$ on every antidiagonal place, i.e. such that $\psi\left(f_{i}, f_{j}\right)=b \cdot \delta_{j, c-i+1}$ (Kronecker-delta) for every $0 \leq i, j \leq c$; let us call this the standard anti-diagonal form over $\mathbb{E}$.

Suppose we have proven that $\psi$ can be put standard anti-diagonal form over $\mathbb{F}$; then we are done with this step: the image of the base $\left\{f_{j} \mid 1 \leq\right.$ $j \leq c\}$ under $g_{i}$ gives an ordered base for $\mathcal{P}_{i}$, for $1 \leq i \leq 2 m$; note that the matrix of $g$ on this $\mathbb{F}$-base equals $\mathbf{1}$; thus for every $\mathcal{P}_{i}$ we obtain a filtration by subvectorspaces with relative quotients of dimension one, and the filtrations are carried into each other under $\mathcal{F}$ respectively $\mathcal{V}$; the equality $\langle x, z=$ $\mathcal{F}(y)>=\langle\mathcal{V}(x), y\rangle^{\sigma}$ shows this gives a final filtration on all subquotients in $A_{\gamma}$, compatible with $\perp$.

We are going to use methods exposed in [26], 29.19. Over $\overline{\mathbb{F}}$ the form $\psi$ can be put in standard anti-diagonal form; let us write $P=\mathcal{P} \otimes_{\mathbb{F}} \overline{\mathbb{F}}$; indeed, if $\operatorname{dim} P>$ 1 , there is an isotropic vector $x \in P$; we can write $P=k \cdot x \oplus k \cdot z \oplus(k \cdot x \oplus k \cdot z)^{\perp}$ with $\psi(x, z) \neq 0$, and we proceed by induction. Moreover the discriminant of this bilinear form is in

$$
\mathbb{E}^{*} / N_{\mathbb{F}} / \mathbb{F}_{p} m\left(\mathbb{F}^{*}\right)=\{1\} .
$$

Let us write $\mathbb{U}$ for the group of linear transformations of $P$ respecting the data (1) (4) above; this is a connected linear group. By [26], page 403 the set of isometry classes of forms isometric with $\psi$ is given by $\mathrm{H}^{1}(\mathbb{F}, \mathbb{U})$; by [55], page 139 we know this cohomology set is trivial; this shows there exists a transformation in $\mathbb{U}(\mathbb{F})$ transforming $\psi$ into the standard anti-diagonal form over $\mathbb{E}$. We have seen that this is enough to construct a final refinement. This ends the construction of a final refinement for $N_{\gamma}$ in the case of an odd cycle $\gamma$

Performing the constructions described above for every cycle, we refine the original filtration to a good filtration with relative steps of dimension one, i.e. 
to a final filtration. This proves Proposition (9.6).

We start with the proof of Theorem (9.4).

(9.7) First step. As $k$ is perfect we can write $N=N_{\text {loc,et }} \oplus N_{\text {loc loc }} \oplus$ $N_{e t, l o c}=N^{\prime} \oplus N_{l o c, l o c}$. Because $k$ is algebraically closed, $N_{l o c, e t} \cong\left(\mu_{p, k}\right)^{f}$, and $N_{e t, l o c} \cong\left((\mathbb{Z} / p)_{k}\right)^{f}$, and we choose a standard base for $N^{\prime}=N_{l o c, e t} \oplus N_{e t, l o c}$. Note that $\operatorname{ES}((G,<,>)$ starts as follows: $\{1,2, \cdots, f, f, \cdots\}$. It suffices to choose a standard base for $\left(M_{l o c, l o c},<,>\right)$. From now on we suppose that $N$ is purely local-local, and hence $\mathcal{F}$ and $\mathcal{V}$ act nilpotently on $A$.

(9.8) We fix some notation, and we recall a result from [27]. In this section a word will be a finite sequence of the letters $\mathcal{F}$ and $\mathcal{V}$. A word will be used in a cyclic way, i. e. putting the first letter as last we will consider the two words as the same. A word is called simple if it is not periodic of period larger than one. We only consider words in which both $\mathcal{V}$ and $\mathcal{F}$ appear. The number of letters in a words will be called the length of the word, indicated by $|w|$.

For a word $w$ we construct, as in [27], a finite group scheme $Z_{w}$ with Dieudonné module $B_{w}=\mathbb{D}\left(Z_{w}\right)$ over $\mathbb{F}_{p}$. If $w=L_{1} \cdots L_{d}$ we choose $z_{1}, \cdots, z_{d}$ plus the convention $z_{d+1}=z_{1}$; if $L_{i}=\mathcal{F}$ we write $\mathcal{F}\left(z_{i}\right)=z_{i+1}$ and $\mathcal{V}\left(z_{i+1}\right)=$ 0 ; if $L_{i}=\mathcal{V}$ we write $\mathcal{V}\left(z_{i+1}\right)=z_{i}$ and $\mathcal{F}\left(z_{i}\right)=0$ (the maps $\mathcal{F}$ are written "clockwise" and $\mathcal{V}$ "anti-clockwise"). This defines the structure of a Dieudonné module on $B_{w}:=\oplus_{i} \mathbb{F}_{p} \cdot z_{i}$ over $\mathbb{F}_{p}[\mathcal{F}, \mathcal{V}]$.

Structure theorem (see [27], Section 5): Let $k \supset \mathbb{F}_{p}$ be an algebraically closed field. Let $N$ be a $B T_{1}$ over $k$. Then there exists a finite set of mutually different simple words $w_{i}$, and integers $n_{i} \in \mathbb{Z}_{>0}$ such that

$$
N \cong \bigoplus_{i}\left(Z_{w_{i}}\right)^{n_{i}} .
$$

This will be called a decomposition into isotypic summands; note that the decomposition as in right hand side above is uniquely determined by $N$, but the isomorphism is far from unique.

Moreover: If $w$ and $w^{\prime}$ are different simple words, a homomorphism $N_{w} \rightarrow$ $N_{w^{\prime}}$ has non-zero kernel and non-zero cokernel.

From a word $w$ we construct its dual $w^{D}$ by replacing $\mathcal{F}$ in $w$ by $\mathcal{V}$ in $w^{D}$ and by replacing $\mathcal{V}$ by $\mathcal{F}$; a word $w$ is called symmetric if $w=w^{D}$ (equality in the cyclic way). Note that $\left(N_{w}\right)^{D}=N_{w^{D}}$ (a canonical isomorphism over $\mathbb{F}_{p}$ ).

(9.9) Second step: Lemma. Let $k$ be an algebraically closed field, an let $(N,<,>)$ a a polarized $\mathrm{BT}_{1}$ over $k$; i.e. $N$ is a $\mathrm{BT}_{1}$ truncated group scheme, 
with Dieudonné module $A=\mathbb{D}(N)$, and $\langle$,$\rangle is a non-degenerate, bilinear,$ alternating form on $A$ satisfying $\langle x, \mathcal{F}(z)\rangle=\langle\mathcal{V}(x), z\rangle^{\sigma}$. Then there exist symmetric simple symmetric words $w_{i}$, integers $n_{i}$, and pairs of non-symmetric words $v_{j}, v_{j}^{D}$ and integers $m_{j}$ and pairings such that

$$
(N,<,>) \cong \bigoplus_{i}\left(Z_{w_{i}},<,>_{i}\right)^{n_{i}} \oplus \bigoplus_{j}\left(Z_{v_{j}} \oplus Z_{v_{j}^{D}},<,>_{j}\right)^{m_{i}} .
$$

Proof. Suppose we write $N=M \oplus P$ in such a way that $M$ and $P$ are sums of isotypic summands of $N$ and such a that for a non-symmetric word $v_{j}$ the summands $Z_{v_{j}}$ and $Z_{v_{j}^{D}}$ both appear either in $M$ or in $P$. The pairing gives a morphism

$$
\left(\begin{array}{cc}
\beta & \gamma \\
\delta & \varepsilon
\end{array}\right)=\zeta: \quad M \oplus P \longrightarrow(M \oplus P)^{D}
$$

Because $<$, > was supposed to be non-degenerate, $\zeta$ is an isomorphism; because of the assumptions on the decomposition $N=M \oplus P$ we see that $\beta$ is an isomorphism (and $\varepsilon$ as well). We define $\varphi:=\beta^{-1} \cdot(-\gamma)$; we define $P^{\prime}=(1+$ $\varphi) P \subset N$; we see that on $N=M \oplus P^{\prime}$ the form is in diagonal shape. As $\langle$, is alternating on $\mathbb{D}(M)$, it is alternating on the summands $\mathbb{D}(M)$ and $\mathbb{D}\left(P^{\prime}\right)$. Induction on the number of isotypic summands in $N$ finishes the proof.

Remark. The lemma just proved is strictly speaking not necessary in the proof below, but it simplifies considerably the notation in the last steps of our proof.

(9.10) In order to apply the structure theorem for $\mathrm{BT}_{1}$ 's to "standard types" we are going to change notation a little bit. The group schemes $Z_{w}$ appearing in the classification by Kraft we are going to replace by group schemes $C_{w}$, more suited to our purpose (and over an algebraically closed field the same up to isomorphism). Let $w$ be a (simple, cyclic) word as above. We define a $\mathrm{BT}_{1} E_{w}$ and its Dieudonné module $C_{w}=\mathbb{D}\left(E_{w}\right)$ almost analogous as before: if $w=L_{1} \cdots L_{d}$ we choose $z_{1}, \cdots, z_{d}$ plus the convention $z_{d+1}=z_{1}$; we write $\left\{z_{1}, \cdots, z_{d}\right\}=\left\{x_{1}, \cdots, x_{b}, y_{1}, \cdots, y_{c}\right\}$ with the convention that "the $y$ 's are images under $\mathcal{V}$ ", i.e. if $L_{j}=\mathcal{V}$, then $z_{j}$ will be baptized $y$, and otherwise $x$; we define $C_{w}:=\oplus_{i} \mathbb{F}_{p} \cdot z_{i}$. If $L_{i}=\mathcal{F}$ we write $\mathcal{F}\left(z_{i}\right)=z_{i+1}$ and $\mathcal{V}\left(z_{i+1}\right)=0$; if $L_{i}=\mathcal{V}$ we write $\mathcal{V}\left(z_{i+1}\right)= \pm z_{i}$ and $\mathcal{F}\left(z_{i}\right)=0$; in fact we write $\mathcal{V}\left(z_{i+1}\right)=+z_{i}$ if $z_{i+1} \in\left\{y_{1}, \cdots, y_{c}\right\}$, and $\mathcal{V}\left(z_{i+1}\right)=-z_{i}$ if $z_{i+1} \in\left\{x_{1}, \cdots, x_{b}\right\}$ (in short: $\mathcal{F}: x \mapsto x, \mathcal{F}: x \mapsto y, \mathcal{V}: y \mapsto+y, \mathcal{V}: x \mapsto-y)$. This constructs the structure of a Dieudonné module on $C_{w}$ over $\mathbb{E}_{p}[\mathcal{F}, \mathcal{V}]$; hence we have constructed a $\mathrm{BT}_{1}$ $E_{w}$.

For a word $w$ we define $a(w)$ to be the number of pairs $\mathcal{F V}$ in the word.

Third step: Lemma. Let $k$ be an algebraically closed field, let $w$ be a word. Then $Z_{w} \otimes k \cong E_{w} \otimes k$. 
Proof. Suppose $\mathbb{D}\left(Z_{w}\right)=\oplus_{i} \mathbb{F}_{p} \cdot z_{i}^{\prime}$ and $\mathbb{D}\left(E_{w}\right)=C_{w}=\oplus_{i} \mathbb{E}_{p} \cdot z_{i}$. Let $|w|=d$ and $a(w)=a$. Consider $z_{i}^{\prime} \mapsto z_{i+1}^{\prime}$ by $\mathcal{F}$, if $L_{i}=\mathcal{F}$ or by $\mathcal{V}^{-1}$ if $L_{i}=\mathcal{V}$; composition of these maps gives $z_{1}^{\prime} \mapsto z_{1}^{\prime}$, and $b \cdot z_{1}^{\prime} \mapsto b^{\sigma^{d}} \cdot z_{1}^{\prime}$ for every $b \in k$. The same process yields $z_{1} \mapsto(-1)^{a} \cdot z_{1}^{\prime}$ in $C_{w}$. Choose $b \in k$ with $b^{\sigma^{d}}=(-1)^{a}$. We define a $k$-linear map

$$
\mathbb{D}\left(C_{w}\right) \otimes k \rightarrow \mathbb{D}\left(Z_{w}\right) \otimes k \quad \text { by } \quad z_{i}^{\prime} \mapsto \beta_{i} \cdot z_{i},
$$

where $\beta_{1}=b$ and, inductively, $\beta_{i+1}=\beta_{i}^{\sigma}$ if $L_{i}=\mathcal{F}$, and $\mathcal{V}\left(\beta_{i+1} \cdot z_{i+1}\right)=\beta_{i} \cdot z_{i}$ if $L_{i}=\mathcal{V}$. This yields $\beta_{d+1}=b^{\sigma^{d}} .(-1)^{a}$. Hence the $k$-linear maps commute with the actions of $\mathcal{F}$ and $\mathcal{V}$, hence we have constructed a Dieudonné module isomorphism $\mathbb{D}\left(C_{w}\right) \otimes k \rightarrow \mathbb{D}\left(Z_{w}\right) \otimes k$. This proves the lemma.

Using the structure theorem, and the two lemmas just proved, in order to prove (9.4) it suffices to show this for isotypic summands:

(9.11) Suppose $k$ is an algebraically closed field of characteristic $p$. Let $(N,[]$,$) be a polarized B T_{1}$ of rank $p^{2 g}$ defined over $k$. Suppose either $N=$ $\left(\left(E_{v} \oplus E_{v D}\right) \otimes k\right)^{n}$, where $v$ is a simple, non-symmetric word, and $n \in \mathbb{Z}_{>0}$ or $N=\left(E_{w} \otimes k\right)^{n}$, where $w$ is a simple symmetric word and $n \in \mathbb{Z}_{>0}$. Suppose $\varphi=\operatorname{ES}(N)$. Then

$$
(N,[,]) \cong\left(N_{\varphi},<,>_{\mathrm{st}}\right) \otimes_{\mathbb{F}_{p}} k .
$$

For the rest of the section we keep notations as in (9.11).

We write $A=\mathbb{D}(N)$. A $k$-base for a Dieudonné module over $k[\mathcal{F}, \mathcal{V}]$ will be called a $(\mathcal{F}, \mathcal{V})$-base if it equals $\left\{z_{1}, \cdots, z_{d}\right\}=\left\{x_{1}, \cdots, x_{b}, y_{1}, \cdots, y_{c}\right\}$ with the conventions as in the definition of $C_{w}$, in particular " $\mathcal{V}: x \mapsto-y$ "; in the situation to be studied we will have $d=2 m$ and $b=m=c$. By the structure theorem and by the lemma in $(9.10)$ we have a $(\mathcal{F}, \mathcal{V})$-base for $A$. On $A$ we have the form [,]. We say that that a base for $A$ is in standard form (with respect to [,]) if it is a $(\mathcal{F}, \mathcal{V})$-base, and if the form [,] is the standard symplectic form on that base. We study all possible $(\mathcal{F}, \mathcal{V})$-bases for $A$ and we show there is at least one which is a standard base for [,].

We will write $z_{i}<z_{j}$ iff $i<j$, i.e. the base vectors in $z_{i}$ are in a strictly lower piece of the canonical stratification

(9.12) Suppose we are in case $N=\left(\left(E_{v} \oplus E_{v} D\right) \otimes k\right)^{n}$ as in (9.11). Then $a(N)$ is even, and we refer to this case as the even case. We shall write $|v|+\left|v^{D}\right|=2 m$

Suppose $w$ is a simple symmetric word; then $a(w)$ is odd; this can be seen as follows; choose a permutation in such a way that $w$ starts with $\mathcal{F}$ and ends with $\mathcal{V}$. From the fact that $w$ is simple and symmetric it follows that $|w|$ is 
even, say $=2 m$, and that $L_{i} \neq L_{i+m}$; hence the first $m$ letters are in the form $\mathcal{F} \cdots \mathcal{F}$ and the last $m$ are $\mathcal{V} \cdots \mathcal{V}$; we see that the number of times that $\mathcal{F} \mathcal{V}$ appears in $w$ is odd. The case $N=\left(E_{w} \otimes k\right)^{n}$ as in $(9.11)$ is called the odd case; here we have $a(N)=a(w) \cdot n$.

On $A$ we have the canonical filtration

$$
0 \subset \cdots \subset A^{(j)} \subset \cdots \subset A
$$

We call $\oplus_{j} A^{(j)} / A^{(j-1)}$ the associated graded of $A$. Note that the canonical filtration has exactly $|v|+\left|v^{D}\right|$, respectively $|w|$ steps. Note that the vectors in $z_{i}$ are in $A^{(j)}$ iff $i \leq j$. The notions of even and odd just explained are the same as the ones used in Section 2.

(9.13) Fourth step: Lemma. We use the notations introduced above, in particular as in (9.11). There exists a base $\left\{z_{1}, \cdots, z_{2 g}\right\}=$ $\left\{x_{1}, \cdots, x_{g}, y_{1}, \cdots, y_{g}\right\}$ which is a $(\mathcal{F}, \mathcal{V})$-base for $A$, which defines a final filtration, and which is in standard form on the associated graded module for the given pairing $[$,$] .$

Proof. We start with a $(\mathcal{F}, \mathcal{V})$-base for $A$ as given in the structure theorem in (9.7) plus the lemma in (9.10). The linear substitutions on $P$ as in Proposition (9.6) can be carried over to the whole $(\mathcal{F}, \mathcal{V})$-base, and we see that we can derive a new $(\mathcal{F}, \mathcal{V})$-base for $A$ which is in standard form on the associated graded module.

From now on we are allowing only substitutions (choices of another base), which leave the residue classes of the base vectors in the associated graded invariant; i.e. a new base vector $Z_{i}^{\prime}$ will be of the form $Z_{i}+$ ? with $? \in A^{(j-1)}$ if $Z_{i} \in A^{(j)}$ and $Z_{i} \notin A^{(j-1)}$.

(9.14) We study the canonical filtration of $N$ as in (9.11). We denote by $z_{j}, x_{i}, y_{i}$ the steps in this filtration, as well as the ordered sets of $n$ base vectors in this step of the filtration (the ones which give non-zero residue classes in that step of the associated graded). As we work with $(\mathcal{F}, \mathcal{V})$-bases, it is clear what is meant by a notation like $\mathcal{F}\left(x_{i}\right)=z_{i}$ : it maps the ordered set of base vectors encoded by $x_{i}$ to the analogous ordered set of base vectors given by $z_{i}$; by $\mathcal{V}\left(x_{i}\right)=y_{j}$ we mean combinatorially that $\mathcal{V}$ is bijective on these steps in the canonical filtration of $A$, and that for base vectors $x_{i}=\left\{x_{i}^{(1)}, \cdots, x_{i}^{(n)}\right\}$, analogously for $y_{j}$, we have that $\mathcal{V}\left(x_{i}^{(t)}\right)=-y_{j}^{(t)}$. When we write $\left[z_{i}, z_{j}\right]$ we mean a $n \times n$-matrix given by the pairings on the pairs of vectors in these sets. 
(9.15) Sets of base vectors as assembled in the canonical filtration on $A$ in consideration will be distributed in 4 subsets. We write

$$
\mathrm{TX}=\left\{x_{i} \mid x_{i} \notin \mathcal{F} A\right\}
$$

(and remember, no base vector in $x_{i}$ is $\mathcal{V} A$ ), and

$$
\mathrm{BX}=\left\{x_{i} \mid x_{i} \in \mathcal{F} A\right\}, \quad \mathrm{X}=\mathrm{TX} \cup \mathrm{BX},
$$

(where $\mathrm{T}$ stand for Top and B stands for Bottom). Further

$$
\mathrm{UY}=\left\{y_{i} \mid y_{i} \notin \mathcal{F} A\right\} \quad \text { and } \quad \mathrm{LY}=\left\{y_{i} \mid y_{i} \in \mathcal{F} A\right\}, \quad \mathrm{Y}=\mathrm{UY} \cup \mathrm{LY}
$$

(Upper and Lower). Note that for $a(w)=a$, respectively $a(v)+a\left(v^{D}\right)=a$ we have TX $=\left\{x_{1}, \cdots, x_{g-a+1}\right\}$ and LY $=\left\{y_{a}, \cdots, y_{1}\right\}$. Note that a Top-X-vector is not an image of any base vector, and a Lower-Y-vector is an image under $\mathcal{F}$ and under $\mathcal{V}$. All other base vectors are an image either under $\mathcal{F}$ (when in $B X$ ), or under $\mathcal{V}$ (when in UY). Every base vector is an image under a power of $\mathcal{F}$ or a power of $\mathcal{V}$ of a \pm Top-X-vector.

For every index $j$, with $1 \leq j \leq a$ we have uniquely determined indices $\xi(j), \eta(j)$, with $1 \leq \xi(j), \quad \eta(j) \leq g-a+1$ (they correspond to Top-Xcoordinates) and positive integers $\overline{f(j)}, v(j)$ such that for base vectors we have:

$$
\mathcal{F}^{f(j)}: x_{\xi(j)} \longmapsto y_{j}, \quad \mathcal{V}^{v(j)}: x_{\eta(j)} \longmapsto-y_{j}, \quad y_{j} \in \mathrm{LY} \quad\left(*_{j}\right) .
$$

Note that for every $i \leq g-a+1$ there exist $j$ and $j^{\prime}$ such that $\xi(j)=i$ and $\eta\left(j^{\prime}\right)=i$.

Claim. $A$ base $\left\{z_{1}, \cdots, z_{2 g}\right\}=\left\{x_{1}, \cdots, x_{g}, y_{1}, \cdots, y_{g}\right\}$ is a $(\mathcal{F}, \mathcal{V})$-base for $A$ iff and only if: the Top-X-base vectors satisfy $\left(*_{j}\right)$ for all $1 \leq j \leq a$ and all base vectors are obtained as a $\mathcal{F}^{?}$-image or a $\mathcal{V}^{?}$-image of a Top-X-base vector.

The proof of this claim follows directly from the definition of a $(\mathcal{F}, \mathcal{V})$-base. Again, note that $z_{j}$ stands for an ordered sequence of $n$ elements; equations should be understood in that sense.

(9.16) We study all possible alternating pairings on $A$, and we are interested to have a base on which the pairings $\left[z_{i}, z_{j}\right]$ are in standard form. We are considering the following combinatorics. Consider the set $\Pi=(\{1, \cdots, 2 m\})^{(2)}=$ $(\{1, \cdots, 2 m\} \times\{1, \cdots, 2 m\}) /(\mathbb{Z} / 2)$ of unordered pairs $\left(z_{i}, z_{j}\right)$. We will define $\Pi_{0} \subset \Pi$, the subset of "zero-classes", and an equivalence relation on $\Pi$ respecting $\Pi_{0} \subset \Pi$, and we consider "1-elements". To this end we write: $(p, q=\mathcal{V}(r)) \prec(\mathcal{F}(p), r)$ if $\mathcal{F} p \neq 0 \neq \mathcal{V} r$, and $\sim$ is the equivalence relation generated by the steps in this partial ordering. We write $(p, q=\mathcal{V}(r)) \in \Pi_{0}$ if $\mathcal{F}(p)=0$; we write $(\mathcal{F}(p), r) \in \Pi_{0}$ if $\mathcal{V}(r)=0$; we write $\left(z_{i}, z_{j}\right) \in \Pi_{0}$ if 
$i+j \leq 2 m$; we denote by $\Pi_{0}$ the set of all classes equivalent with any of the previously mentioned zero classes; in particular we see that $(\mathcal{V}(p), \mathcal{V}(r)) \in \Pi_{0}$ and $(\mathcal{F}(p), \mathcal{F}(r)) \in \Pi_{0}$; note that $A^{(i)}$ and $A^{(j)}$ pair to zero if $i+j \leq 2 m$, hence:

Observation. If $[$,$] is an alternating pairing on A$, and $\left(z_{i}, z_{j}\right) \in \Pi_{0}$ then $\left[z_{i}, z_{j}\right]=0$. This follows from $[p, q=\mathcal{V}(r)]^{\sigma}=[\mathcal{F}(p), r]$.

Observation. Suppose we have non-zero classes $\left(z_{i}, z_{j}\right) \prec(p, q)$. Then either $z_{i} \in \mathrm{X}$ and $z_{j} \in \mathrm{Y}$ or conversely. Indeed, we can only "move on" if at least one of the elements is in the image of $\mathcal{V}$, and as the element is non-zero, the other is not in the image of $\mathcal{V}$.

An element of the form $\left(x_{j}, y_{j}\right)$ is called a 1-element.

Observation. If $[$, ] is a non-degenerate alternating pairing which is in standard form on the associated graded, then $\left[x_{j}, y_{j}\right]=1$ (i.e. the diagonal matrix of size $n \times n) \forall j$.

Basic observation. If in $\Pi$ we have equivalent pairs $(p, q) \sim(r, s)$ then $[p, q]=$ $0 \Leftrightarrow[r, s]=0$.

Claim. (i) All 1-elements form one equivalence class.

(ii) Let $\left\{\pi_{1} \prec \cdots \prec \pi_{e}\right\} \subset \Pi$ be a longest chain of non-zero, non-1 equivalent elements, $\pi_{j}=\left(z_{j}^{\prime}, z_{j}^{\prime \prime}\right)$; then $z_{1}^{\prime}$ does not occur in $\pi_{2}, \cdots, \pi_{e}$ (i.e. there are no "cyclic" equivalence classes in the case of non-zero and non-1-classes).

(iii) Let $\left\{\pi_{1} \prec \cdots \prec \pi_{e}\right\} \subset \Pi$ be a longest chain of non-zero and non-1 equivalent elements. Then $\pi_{1}=\left(x_{i}, z_{j}\right)$ with $x_{i} \in \mathrm{TX}$ and $z_{j} \in \mathrm{TX} \cup \mathrm{UY}$.

(iv) Let $\delta+1=\#\left(\left(\Pi-\Pi_{0}\right) / \sim\right)$ be the number of equivalence classes of nonzero elements. Consider $A$ with a $(\mathcal{F}, \mathcal{V})$-base for $A$. The set $\mathcal{Q}$ of alternating non-degenerate pairings on $A$ which are in standard form on the associated graded equals $k^{\delta \cdot n^{2}} \cong \mathcal{Q}$ (and the bijection will be indicated in the proof).

Proof. (i) In the situation (9.11) all 1-elements form a single equivalence class: consider separately the symmetric word $w$ or the pair of words $v$ and $v^{D}$.

(ii) Suppose there would exist a cyclic equivalence class which consists of non-zero and non- 1 elements. In case of $w$ there would be an integer $q$, say $0<q<2 m$, such that $L_{i} \neq L_{i+q}$ for all $i$. As $w$ is simple this can only happen if $q=m$. The case $v$ and $v^{D}$ follows in the same way (because $v$ is simple and non-symmetric).

(iii) There is an element "before" $(p, q)$ if one of the $p, q$ is an image under $\mathcal{F}$. So the left end $(p, q)$ of a non-zero, non-1 equivalence class has elements not in the image of $\mathcal{F}$, not both in the image of $\mathcal{V}$.

(iv) Let $\left(x^{(1)}, z^{(1)}\right), \cdots,\left(x^{(\delta)}, z^{(\delta)}\right)$ be the left-ends of the non-zero and non- 1 equivalence classes. Any [, ] as in (iv) defines $n \times n$-matrices $M_{1}, \cdots, M_{\delta} \in$ $k^{n^{2}}$ by $\left[x^{(j)}, z^{(j)}\right]=: M_{j}$; for this choice we take once and for all an ordering for each pair $\left(x^{(j)}, z^{(j)}\right)$. Conversely, given such matrices, we define $\left[x_{i}, y_{i}\right]=1$, and $\left[x^{(j)}, z^{(j)}\right]=M_{j}$; this defines all pairings between base-vectors, and we check that this gives a [,] as in (iv). This finishes the proof of the claim. 
We write $\Pi^{0}:=\Pi-\Pi_{0}-\Pi_{1}$ for the set of non-zero and non-1-elements. Conclusion: The evaluation map $\mathcal{Q} \rightarrow k^{\delta \cdot n^{2}}$, given by computing [, ] on left ends of equivalence classes in $\Pi^{0}$, is bijective. We will consider the set $\mathcal{Q}$ as $\mathbb{A}^{\delta \cdot n^{2}}(k)$ and treat it as a variety over $k$.

(9.17) We consider the group $\mathcal{S}$ of all $k$-linear transformations of $A$ which are Dieudonné module isomorphisms, and which map a given $(\mathcal{F}, \mathcal{V})$-base to a $(\mathcal{F}, \mathcal{V})$-base. It acts on the set $\mathcal{Q}$ of non-degenerate alternating forms on $A$ (by conjugation): $\mathcal{S} \times \mathcal{Q} \rightarrow \mathcal{Q}$; this will be considered as the action of a linear group over $k$ onto a variety over $k$ (identifying a linear group over $k$ with the group of its $k$-rational points, etc.). We are going to prove that the orbit of every element of $\mathcal{Q}$ under $\mathcal{S}$ is the whole of $\mathcal{Q}$ (and hence we can change [,] into $<,>_{\text {st }}$ ). We see that in general the stabilizer of an element of $\mathcal{Q}$ is non-trivial. We give the proof by considering an algebraic subvariety $L \subset \mathcal{S}$ containing the identity of this algebraic group, and showing that the differential of the action parameterized by $L$ maps surjectively onto the tangent space at $[,] \in \mathcal{Q}$.

Remark: The dimension of $\mathcal{S}$ in general is much larger that the dimension of $\mathcal{Q}$, but that information is not of much help, because in general the stabilizer in $\mathcal{S}$ of an element of $\mathcal{Q}$ is non-trivial.

(9.18) Description of $\mathcal{S}$ and of $L$. We introduce "variables" $\beta_{s, j}$ and $\gamma_{s, t}$, each is a $n \times n$-matrix (indices will be specified below). Given $A$ as above with a $(\mathcal{F}, \mathcal{V})$-base we write:

$$
x_{s}^{\prime}=x_{s}+\sum_{j<s} \beta_{s, j} \cdot x_{j}+\sum_{y_{t}<x_{s}} \gamma_{s, t} \cdot y_{t}, \quad \forall x_{s} \in \mathrm{TX} \quad(* *) ;
$$

these are subject to the relations $\left(*_{j}\right)$ as explained in $(9.15)$; we write $(*)$ for the set $\left(*_{j}\right) \forall j$. We define vectors $z_{i}^{\prime}$ by: taking all images under powers of $\mathcal{F}$ and powers of $\mathcal{V}$, and deleting the ones which are zero. The new base vectors in the sequel will be denoted by $\left\{\rho\left(z_{i}\right)\right\}$ once all $\beta_{s, j}$ and $\gamma_{s, t}$ satisfying $(*)$ (and perhaps several conditions).

Claim. The substitutions given by (**) subject to $(*)$ form a group which are all substitution which are the identity on the associated graded, and which map the given $(\mathcal{F}, \mathcal{V})$-base for $A$ to a $(\mathcal{F}, \mathcal{V})$-base. This defines a group scheme.

Remark. In fact, this group scheme in general is non-reduced. This will play no role later in our considerations. We are going to define a subvariety $L \subset \mathcal{S}$ by imposing extra conditions on the variables.

(9.19) For a set $z_{j}$ of base vectors we define $d\left(z_{j}\right)=z_{2 m+1-j}$; note that $d\left(x_{i}\right)=y_{i}$ and $d\left(y_{i}\right)=x_{i}$. We consider the set $\Lambda$ of ordered pairs $\left(z_{i} \& z_{j}\right)$, such that $z_{j}<z_{i}$ :

$$
\Lambda=\left\{\left(z_{i} \& z_{j}\right) \in(\{1, \cdots, 2 m\})^{2} \mid z_{j}<z_{i}\right\} .
$$


We write $\Lambda_{1}=\{(z \& d(z))\}$, and we call elements of this set "1-elements". We define

$$
D: \Lambda \rightarrow \Pi \quad \text { by } D\left(z_{i} \& z_{j}\right):=\left(z_{i}, d\left(z_{j}\right)\right) .
$$

We write $\Lambda_{0}:=D^{-1}\left(\Pi_{0}\right)$.

Claim. The map $\Lambda-\Lambda_{1} \rightarrow \Pi-\Pi_{1}$ is 2 -1-surjective.

In fact, $\left(z^{\prime}, z^{\prime \prime}\right)$ is the image of $\left(z^{\prime}, d\left(z^{\prime \prime}\right)\right)$ if $d\left(z^{\prime \prime}\right)<z^{\prime}$ respectively of $\left(z^{\prime \prime}, d\left(z^{\prime}\right)\right)$ if $d\left(z^{\prime}\right)<z^{\prime \prime}$; hence $D: \Lambda \rightarrow \Pi$ is surjective. On $\Lambda-\Lambda_{1}$ the map $\left(z^{\prime} \& z^{\prime \prime}\right) \mapsto$ $\left(d\left(z^{\prime \prime}\right) \& d\left(z^{\prime}\right)\right)$ is fixed point free with quotient $\Pi-\Pi_{1}$.

On $\Lambda$ we define an equivalence relation, indicated by $\approx$; we write $\left(z^{\prime} \& z^{\prime \prime}\right) \prec$ $\left(\mathcal{F} z^{\prime} \& \mathcal{F} z^{\prime \prime}\right)$ if $\mathcal{F} z^{\prime} \neq 0 \neq \mathcal{F} z^{\prime \prime}$; we write $\left(\mathcal{V} z^{\prime} \& \mathcal{V} z^{\prime \prime}\right) \prec\left(z^{\prime} \& z^{\prime \prime}\right)$ if $\mathcal{V} z^{\prime} \neq 0 \neq$ $\mathcal{V} z^{\prime \prime}$; a partial ordering, and the equivalence relation given by this is the one generated by iterating these definitions.

Claim. We have:

$$
\left(z^{\prime} \& z^{\prime \prime}\right) \prec\left(u^{\prime} \& u^{\prime \prime}\right) \quad \Longleftrightarrow \quad D\left(z^{\prime} \& z^{\prime \prime}\right) \prec D\left(u^{\prime} \& u^{\prime \prime}\right) .
$$

In fact, $d\left(z^{\prime \prime}\right)= \pm \mathcal{V}\left(d\left(\mathcal{F} z^{\prime \prime}\right)\right.$, hence $\left(z^{\prime} \& z^{\prime \prime}\right) \prec\left(\mathcal{F} z^{\prime} \& \mathcal{F} z^{\prime \prime}\right)$ implies $\left(z^{\prime}, \mathcal{V}\left(d\left(\mathcal{F} z^{\prime \prime}\right) \prec\left(\mathcal{F} z^{\prime}, d\left(\mathcal{F} z^{\prime \prime}\right)\right)\right.\right.$, and conversely. An analogous statement with $\left(\mathcal{V} z^{\prime} \& \mathcal{V} z^{\prime \prime}\right) \prec\left(z^{\prime} \& z^{\prime \prime}\right)$ proves the claim.

We number the $\sim$-equivalence classes $E_{1}, \cdots, E_{\delta} \subset \Pi-\Pi_{0}-\Pi_{1}$. We define variables $T_{1}, \cdots, T_{\delta}$, each is a $n \times n$-matrix of variables, and we are going to define a morphism

$$
\operatorname{Spec}\left(k\left[T_{1}, \cdots, T_{\delta}\right]\right) \rightarrow \mathcal{S}
$$

the image of this maps will be a closed, reduced subvariety $L \subset \mathcal{S}$; we study the action

$$
L \times \mathcal{Q} \rightarrow \mathcal{Q} .
$$

Note that for every $\sim$-equivalence class $E \subset \Pi^{0}$ there are exactly two disjoint $\approx$-equivalence classes $E^{\prime}, E^{\prime \prime} \subset \Lambda^{0}$ such that

$$
D: E^{\prime} \stackrel{\sim}{\longrightarrow} E, \quad D: E^{\prime \prime} \stackrel{\sim}{\longrightarrow} E .
$$

We consider an $\sim$-equivalence class $E \subset \Pi-\Pi_{0}$. There are three possibilities:

(1) The class contains $\left(x_{i}, y_{i}\right)$ for some index $i$. In this case there is only one $\sim$-equivalence class, it contains all $\left(x_{j}, y_{j}\right)$. This is what we called the 1-equivalence-class.

(2) The left end of $E$ equals $\left(x_{s}, x_{t}\right)$ with $x_{s}, x_{t} \in$ TX. In this case $E$ consists of one element: $E=\left\{\left(x_{s}, x_{t}\right)\right\}$. 
(3) The left end of $E$ equals $\left(x_{s}, z\right)$ with $z \in \mathrm{UY}$.

For zero classes we define:

$D\left(x_{s} \& z\right) \in \Pi_{0} \quad \Longrightarrow \quad \beta_{s, j}=0$, if $z=x_{j}$, respectively $\gamma_{s, t}=0$, if $z=y_{t}$

Suppose we are in case (2). Then $D\left(x_{s} \& y_{t}\right)=\left(x_{s}, x_{t}\right)=D\left(x_{t} \& y_{s}\right)$. Assume that $t<s$ (otherwise interchange); then $y_{s}<y_{t}<x_{s}$. We write:

$$
x_{s} \in \mathrm{TX}, \quad y_{t}<y_{s} \quad \Longrightarrow \quad \gamma_{t, s}=0 \quad(\mathrm{~L} 1)
$$

This condition will remove certain ambiguities. If $E_{i}=E=\left\{\left(x_{s}, x_{t}\right)\right\}=$ $\left\{x_{s(i)}, x_{t(i)}\right\}$ with $x_{s}, x_{t} \in \mathrm{TX}$ and $x_{t}<x_{s}$, hence $y_{s}<y_{t}<x_{s}$, we define:

$$
T_{i}=: \gamma_{s(i), t(i)}=\gamma_{s, t}
$$

Suppose we are in case (3). We write $(x, z) \in E \subset \Pi^{0}$ for its left end; hence $x \in \mathrm{TX}$, and $z \in \mathrm{UY}$. Note that $d(z)<x$ and $d(x)<z$ hence $D(x \& d(z))=$ $(x, z)=D(z \& d(x))$. We choose the notation for $E^{\prime}, E^{\prime \prime}$ mapping onto $E$ in such a way that $\left(x \& d(z) \in E^{\prime}\right.$ and $(z \& d(x)) \in E^{\prime \prime}$.

If $E=E_{i}$ with left end $\left(x_{s(i)}, z_{u(i)}\right)$ we define

$\beta_{s(i), j}=T_{i}$, if $d\left(z_{u(i)}\right)=x_{j} \quad$ respectively $\quad \gamma_{s, t}=T_{i}$, if $d\left(z_{u(i)}\right)=y_{t}$

we follow the ordered set $E_{i}^{\prime}$, and we define $\beta_{s, j}= \pm T_{i}^{p^{c}}$ respectively $\gamma_{s, t}=$ $\pm T_{i}^{p^{c}}$ with the appropriate sign, and with the power of $p$ according to the number of steps $c$ in $E_{i}^{\prime}$ that is $\left(x_{s} \& x_{j}\right) \in E_{i}^{\prime}$ respectively $\left(x_{s} \& y_{t}\right) \in E_{i}^{\prime}$ from the left end side of $E_{i}^{\prime}$.

All elements appearing in $E^{\prime \prime}$ give rise to variables which we equate to zero:

$$
\left(x_{s} \& x_{j}\right) \in E^{\prime \prime} \Longrightarrow \beta_{s, j}=0, \quad\left(x_{s} \& y_{t}\right) \in E^{\prime \prime} \Longrightarrow \gamma_{s, t}=0
$$

The relations $(*),(\mathrm{L} 0),(\mathrm{L} 1),(\mathrm{L} 2),(\mathrm{L} 3)$ and (L4) define the value of all $\beta_{s, j}$ and $\gamma_{s, t}$ introduced in (9.18); under these conditions we have defined

$$
\operatorname{Spec}\left(k\left[T_{1}, \cdots, T_{\delta}\right) \stackrel{\sim}{\longrightarrow} L \subset \mathcal{S} .\right.
$$

We see that indeed this defines a closed subvariety of $\mathcal{S}$. 
(9.20) Fifth step: Proposition. Equating all variables $T_{i}$ to zero gives $1 \in L \subset \mathcal{S}$, the identity transformation. Choose any $[,] \in \mathcal{Q}$ as in (9.11). The action $L \times \mathcal{Q} \rightarrow \mathcal{Q}$ at [,] gives a morphism $L \rightarrow L \cdot[$,$] . The differential of the$ action $L \times \mathcal{Q} \rightarrow \mathcal{Q}$ at [,] is a surjective linear map $\mathfrak{t}_{L, 1} \rightarrow t_{\mathcal{Q},[,]}$. It follows that the orbit of [,] under the action $\mathcal{S} \times \mathcal{Q} \rightarrow \mathcal{Q}$ equals $\mathcal{S} \cdot[]=,\mathcal{Q}$.

Proof. We write $I=\left(T_{i} \mid i\right) \subset k\left[T_{1}, \cdots, T_{\delta}\right]=: k[T]$ for the ideal generated by these variables. We compute the action of $\operatorname{Spec}\left(k[T] / I^{2}\right)$ on $\mathcal{Q}$. Let us write $\tau_{i}=T_{i} \bmod I^{2}$. We see that all elements $\beta_{s, t}$ and $\gamma_{s, t}$ not defined by a left end are in $I^{2}$.

Claim. Under the action of $\operatorname{Spec}\left(k[T] / I^{2}\right)$ on $\mathcal{Q}$ the matrices $M_{j}=\left[z_{j}^{\prime}, z_{j}^{\prime \prime}\right]$ are transformed into $M_{j}+ \pm \tau_{j}$; in fact, let us write $\left\{\rho\left(z_{i}\right)\right\}$ as in (9.15); then we compute $\rho \cdot M_{j}=\left[\rho\left(z_{j}^{\prime}\right), \rho\left(z_{j}^{\prime \prime}\right)\right]$; in case (2), using (L0) and (L2) and using $I^{2}=0$ this gives:

$\left[\rho\left(x_{s}\right), \rho\left(x_{t}\right)\right]=\left[x_{s}+T_{i} \cdot y_{t}+\sum_{j<s} \beta_{s, j} \cdot x_{j}+\sum_{y_{u}<x_{s}, u \neq t} \gamma_{s, u} \cdot y_{u}, \quad x_{t}+0 \cdot y_{s}+\cdots\right]=\left[x_{s}, x_{t}\right]+ \pm \tau_{i} ;$

in case (3), if $(x, z) \in E_{i}$ is the left end, using (L3) and (L4) and $I^{2}=0$ in case $(x, z)=\left(x_{s}, y_{j}\right)$ we obtain:

$$
[\rho(x), \rho(z)]=\left[x_{s}+T_{i} \cdot x_{j}+\sum_{u<s, u \neq j} \beta_{s, u} \cdot x_{u}+\sum_{y_{t}<x_{s}} \gamma_{s, t} \cdot y_{t}, \quad y_{j}+0 \cdot y_{s}+\cdots\right]
$$

respectively if $(x, z)=\left(x_{s}, x_{t}\right)$ we obtain:

$$
=\left[x_{s}+T_{i} \cdot y_{t}+\cdots, \quad x_{t}+0 \cdot y_{s}+\cdots\right] .
$$

This proves the claim.

Hence it proves that $\mathfrak{t}_{L, 1} \rightarrow \mathfrak{t}_{\mathcal{Q} \text { [.] }}$ is surjective. Hence for every [,] $\in \mathcal{Q}$ the set $L \cdot[,] \subset \mathcal{Q}$ is dense. We know that $\mathcal{Q}$ is irreducible. This proves that $\mathcal{S}$ acts transitively on $\mathcal{Q}$.

In the basic observation in (9.16) we see that pairings between base vectors (non dual ones) are zero iff these parings are zero on all left ends of equivalence classes. The previous proposition shows that, starting from (9.11) there is a transformation $\left\{x_{s} \mapsto x_{s}^{\prime} \mid x_{s} \in \mathrm{TX}\right\}=\rho \in \mathcal{S}(k)$ such that $\rho \cdot[]=,[\rho(), \rho()]$ is zero on left ends of non-1- -equivalence classes. Hence $\rho \cdot[]=,<,>_{\text {st }}$. This proves the claim as in (9.11). By what has been said and proven in the first three steps, this proves Theorem (9.4).

(9.21) Remark. By [59], Proposition 2.4.14 we know that every orbit of a unipotent group $\mathcal{S}_{\text {red }}$ acting on an affine variety $\mathcal{Q}$ is closed; hence we see that in the proof of (9.20) it suffices to compute the tangent action of $L$ at $\langle\text {, }\rangle_{\text {st }}$ in order to prove that every $[$,$] is in the orbit \mathcal{S} \cdot\langle,\rangle_{\text {st }}$. 
(9.22) Remark. Suppose a final filtration on a polarized $\mathrm{BT}_{1}$ truncated group scheme is given. We note there are in general many choices for a standard base matching this filtration. here is one example. Choose indices $i$ and $j$ with $n_{j}<m_{i}$; then $n_{i}=2 g+1-m_{i}<2 g+1-n_{j}=m_{j}$; we choose some $\beta$ in the base field $k$, we change $X_{i}$ to $X_{i}^{\prime}=X_{i}+\beta \cdot Y_{j}$ and $X_{j}$ to $X_{j}^{\prime}=X_{j}+\beta \cdot Y_{i}$, and we leave all other base vectors the same. Clearly the new set is a standard base matching the same filtration. Examples of a different nature are easy to give, as we have seen in the proof above: in general there are many isomorphisms on $A_{\varphi}$ which by conjugation do not change $<$, $\rangle_{\text {st }}$.

\section{Moving in a stratum}

In this section we fix an elementary sequence $\varphi \in \Phi$, and we prove:

(10.1) Proposition. Every component of $S_{\varphi}$ has dimension at least $|\varphi|$. Later we will show in fact that:

$$
\operatorname{dim}\left(S_{\varphi}\right)=|\varphi|
$$

Let $x \in S_{\varphi}(k)$, where $k$ is an algebraically closed field. We are going to study deformation theory around $\left[\left(X_{0}, \lambda_{0}\right)\right]=x_{0} \in \mathcal{A}$, especially a part which "stays inside $S_{\varphi}$ ". We use notations introduced earlier, especially we use a standard base for $\mathbb{D}\left(\left(X_{0}, \lambda_{0}\right)[p]\right)$ as described in Section 9 . We write $\psi$ for the final sequence associated with $\varphi$, and we suppose we have chosen, see (9.6), a final filtration

$$
A_{0} \subset A_{1} \subset \cdots \subset A_{g}=\mathcal{F}(A) \subset \cdots \subset A_{2 g}=A=\mathbb{D}\left(N_{0}\right) .
$$

The standard base $\left\{Z_{1}, \cdots, Z_{g}\right\}=\left\{X_{1}, \cdots, X_{g}, Y_{g}, \cdots, Y_{1}\right\}$ for $A$ is lifted to a symplectic base $\left\{X_{1}^{\prime}, \cdots, X_{g}^{\prime}, Y_{g}^{\prime}, \cdots, Y_{1}^{\prime}\right\}$ for $M=\mathbb{D}\left(X_{0}\left[p^{\infty}\right]\right)$ such that $Y_{i}^{\prime} \in$ $\mathcal{F}(M)$.

We use the integers $\left\{n_{g}, \cdots, n_{1}\right\}$ and $\left\{m_{1}, \cdots, m_{g}\right\}$ as introduced in Section 9. We write

$$
I_{\varphi}=I=\left\{n_{g}, \cdots, n_{g-a-1}\right\} \quad \text { and } \quad J_{\varphi}=J=\left\{m_{1}, \cdots, m_{g-a}\right\} ;
$$

this corresponds with the $Y_{j}$, respectively $X_{i}$, contained in $A_{g}=\mathcal{F}(A)$, where $a=a\left(X_{0}\right)=a(\varphi)=g-\varphi(g)$. We introduce variables $t_{u, v}$, some of which are put to zero, some of which are considered as variables, and in this way we are going to construct a ring $R_{\varphi}$. To this end we take subset $D_{\varphi} \subset\{1, \cdots, g\} \times\{1, \cdots, g\}$ defined as follows:

(J) it consists of all $(u, v)$ such that $u \geq v>a$ and

(I) it consists of all $(u, v)$ such that $1 \leq v \leq a$ and $m_{g-u+1} \leq n_{g-v+1}$. 


\section{(10.2) Observation.}

$$
\#\left(D_{\varphi}\right)=|\varphi| .
$$

Indeed, $\sum_{j \in J} \varphi(j)$ equals the number of pairs in (J) above, and $\sum_{i \in I} \varphi(i)$ equals the number of pairs in (I) above.

We define

$$
R_{\varphi}=k\left[\left[t_{u, v} \mid(u, v) \in D \quad \text { or } \quad(v, u) \in D\right]\right] /\left(t_{u, v}-t_{v, u}\right) .
$$

Note that if $(u, v)$ in $(\mathrm{I})$ above then it is not in $(\mathrm{J})$, and $(v, u)$ is not in $(\mathrm{J})$. Note that the Krull dimension of this ring equals $|\varphi|$. We write $T_{(u, v)} \in W_{\infty}\left(R_{\varphi}\right)$ for the Teichmüller lift of $t_{u, v} \in R_{\varphi}$, and we write $T=\left(T_{u, v} \mid 1 \leq u, v \leq v\right)$ for the corresponding matrix.

The display of $M$ on the symplectic $W$-base $\left\{X_{1}^{\prime}, \cdots, X_{g}^{\prime}, Y_{g}^{\prime}, \cdots, Y_{1}^{\prime}\right\}$ for $M$ we denote by

$$
\left(\begin{array}{ll}
A & B \\
C & D
\end{array}\right)
$$

(with apologies that we use the symbol $A$ with two different meanings; we expect there will be no confusion). With the matrix $(T)$ defined above we obtain a display

$$
\left(\begin{array}{cc}
A+T C & B+T D \\
C & D
\end{array}\right) .
$$

This defines a quasi-polarized $p$-divisible group over $\operatorname{Spec}\left(R_{\varphi}\right)$ deforming $\left(X_{0}\left[p^{\infty}\right], \lambda_{0}\right)$; hence by the theorem of Serre and Tate, see [30], [22], [32] , we obtain a principally polarized abelian scheme $\left(X^{(T)}, \lambda\right) \rightarrow \operatorname{Spec}\left(R_{\varphi}\right)$; we write $\left[\left(X^{(T)}, \lambda\right)\right]=x \in \mathcal{A}\left(R_{\varphi}\right)$ for the corresponding point.

(10.3) Claim. We have $\left[\left(X^{(T)}, \lambda\right)\right]=x \in S_{\varphi}\left(R_{\varphi}\right)$.

In fact, if $\psi\left(m_{i}\right)=n_{s}$, then

$$
\mathcal{F}^{(T)}\left(X_{i}^{\prime}\right)=\sum T_{j, g-s+1} \cdot X_{g-j+1}^{\prime}+Y_{s}^{\prime} .
$$

The variables coming from (J) above show up only multiplied by a factor $p$. These two fact show that this bases induces a filtration on $A^{(T)}$ on which the elementary sequence $\varphi$ is realized, say over a perfect closure of the field of fractions of $R_{\varphi}$. This proves the claim.

Hence we have constructed $x_{0} \in \operatorname{Spec}\left(R_{\varphi}\right) \subset S_{\varphi}$. By the observation above this proves the proposition. 


\section{Moving out of a stratum}

In this section we consider elementary sequences $\varphi$ and $\varphi^{\prime}$ such that

$$
\varphi \prec \varphi^{\prime} \quad \text { and }|\varphi|+1=\left|\varphi^{\prime}\right| \text {. }
$$

This means there is an index $b$ with $0<b \leq g$ and

$$
\varphi(i)=\varphi^{\prime}(i) \text { for } i \neq b, \quad 0<i \leq g, \quad \text { and } \varphi(b)+1=\varphi^{\prime}(b) .
$$

Note that this is only possible when $\varphi(b-1)=\varphi(b)<\psi(b+1)$.

\section{Proposition. Under these conditions}

$$
S_{\varphi^{\prime}} \subset\left(S_{\varphi}\right)^{c} .
$$

Proof. It suffices to prove the case that $\varphi(1)=0$, which we suppose from now on. As in the previous section we write: $\left[\left(X_{0}, \lambda_{0}\right)\right]=x_{0} \in S_{\varphi} \subset \mathcal{A}$ and $\left\{Z_{1}, \cdots, Z_{2 g}\right\}=\left\{X_{1}, \cdots, X_{g}, Y_{g}, \cdots, Y_{1}\right\}$, a standard basis for $A$ is lifted to a symplectic base $\left\{X_{1}^{\prime}, \cdots, X_{g}^{\prime}, Y_{g}^{\prime}, \cdots, Y_{1}^{\prime}\right\}$ for $M=\mathbb{D}\left(X_{0}\left[p^{\infty}\right]\right)$ such that $Y_{i}^{\prime} \in$ $\mathcal{F}(M)$. Note that $\varphi(b-1)=\varphi(b)<\psi(b+1)$; hence $Z_{b}$ is one of the base vectors $Y_{?}$, in fact $Z_{b}=Y_{y}$ with $y=g-b+\varphi(b)$; moreover $Z_{b+1}$ is one of the base vectors $X_{?}$, in fact $Z_{b+1}=X_{x}$ with $x=\varphi(b+1)$. On the module $A$ we have $\mathcal{F}\left(X_{b}\right)=Y_{y}$. We define $u=g+1-\varphi(b+1)$, and $v=b+1-\varphi(b)$; we write $t_{u, v}=q$, a variable over $k$, and $t_{v, u}=q$, and we put all other $t_{i, j}=0$; the Teichmüller lift we denote by $Q \in W_{\infty}(k[[q]])$ (and all others equal to 0$)$, thus obtaining a matrix $T$. From this we obtain a deformation $\left(X^{(T)}, \lambda\right) \rightarrow \operatorname{Spec}(k[[q]])$.

Claim. For the generic fiber, we have $\operatorname{ES}\left(X_{\eta}^{(T)}, \lambda\right)=\varphi^{\prime}$.

Observe that $Y_{y} \in \mathcal{F}(A)$, hence $y \geq g-a+1$, hence $v \leq a$. In the deformation we see that $T_{v, u}$ is multiplied by a factor $p$. We conclude that:

$\mathcal{F}^{(T)}\left(X_{b}\right)=Y_{y}+T \cdot X_{x}$, and

$\mathcal{F}^{(T)}\left(Z_{i}\right)=\mathcal{F}\left(Z_{i}\right)$ for all $i \neq m_{b}$, i.e. $Z_{i} \neq X_{b}$.

Let $K$ be a perfect field containing $k[[q]]$, write $(X, \lambda)=\left(X^{(T)}, \lambda\right)$, and let us show that $\operatorname{ES}(X, \lambda)=\varphi^{\prime}$.

In case $1 \leq b<g$ the old standard base is

$$
\left\{Z_{1}, \cdots, Z_{b-1}, Y_{y}, X_{x}, Z_{b+2}, \cdots, Z_{g-b-2}, X_{y}, Y_{x}, Z_{g-b+1}, \cdots, Z_{2 g}\right\} .
$$

We choose a new base for the module on which the display is defined by choosing $\left\{Z_{1}, \cdots, Z_{b-1}, Y_{y}+T \cdot X_{x}, Y_{y}, Z_{b+2}, \cdots, Z_{g-b-2}, T X_{y}+Y_{x}, Y_{x}, Z_{g-b+1}, \cdots, Z_{2 g}\right\}$. This ordered set defines a filtration; with the $\sigma$-linear map given by $\mathcal{F}^{(T)}\left(X_{b}\right)=$ $Y_{y}+T \cdot X_{x}$, etc. as above, we obtain a final filtration with elementary sequence $\varphi^{\prime}$.

In case $b=g$, we have $x=g-a+1=y$; the old standard base is $\left\{Z_{1}, \cdots, Z_{b-1}, Y_{y}, X_{x}, Z_{b+2}, \cdots, Z_{2 g}\right\}$, and on the new base given by the ordered set $\left\{Z_{1}, \cdots, Z_{b-1}, Y_{y}+T \cdot X_{x}, Y_{y}, Z_{b+2}, \cdots, Z_{2 g}\right\}$ we obtain a final filtration with elementary sequence $\varphi^{\prime}$. This proves the claim, and it finishes the proof of the proposition. 
(11.2) Corollary. For every $\varphi \in \Phi$ we have:

$$
\operatorname{dim}\left(S_{\varphi}\right)=|\varphi| .
$$

In fact, if $(g(g+1) / 2)-|\varphi|=c$, there is a sequence

$$
\varphi=\varphi_{0} \supsetneqq \varphi_{1} \supsetneqq \cdots \supsetneqq \varphi_{c}=\{1, \cdots, g\} .
$$

By the previous corollary this shows that every component of $S_{\varphi}$ has dimension at most $|\varphi|$. By the main result of the proceeding section the opposite inequality holds, and we are done.

Remark. It seems strange that we obtain a regular deformation space starting at a point in the boundary of $S_{\varphi^{\prime}}$, although we know that in general a stratum is "very singular" at its boundary. However note that we consider deformations respecting a final filtration. For example, if we start in a point of $\Sigma$, and and we want to move into the interior of $L$, there are many ways of doing that. But what we proved, implies that giving a final filtration for the closed fiber, this singles out a "direction of deformation" into $L$.

\section{Transport of structure along the boundary of a stratum}

In this section we prove that the boundary of a stratum $S_{\varphi}$ is a union of (lower dimensional) strata. We use Lemma (12.4), and give the proof in Proposition (12.5). In (12.1) we explain some notation. The results (12.2) and (12.3) will not be used in the sequel; these are included in order to explain the complexity of the situation; we expect that (12.3) can be of independent interest. The main result of this section is not difficult if we work over fields of characteristic $p>2$; in such a case an anti-symmetric pairing on $N$ gives an alternating pairing on $A:=\mathbb{D}(N)$; in that case a proof of Proposition (12.5) is not so difficult. We have chosen to include also the case $p=2$ in the general proof, leaving simplifications in other cases to the reader, but indicating how biextensions can be brought in.

\section{(12.1) Polarizations and biextensions.}

(AV) Let $(\mathcal{X}, \lambda) \rightarrow S$ be a polarized abelian scheme. By [54], 1.4 on page 224, we know:

$$
\operatorname{Biext}^{1}\left(X_{1}, X_{2} ; \mathbb{S}_{m}\right) \stackrel{\sim}{\longrightarrow} \operatorname{Hom}\left(X_{1}, X_{2}\right)
$$

(we will write $X_{1}=X=X_{2}$, numbering the copies of $X$ involved in order to keep track what is what). The polarization is an isogeny $\lambda: X \rightarrow X^{t}$. We know that $\lambda$ is symmetric in the sense that

$$
\left(\lambda: X_{1} \rightarrow X_{2}^{t}\right)=\left(\lambda^{t}:\left(X_{2}\right)^{t t}=X_{2} \rightarrow X_{1}^{t}\right) ;
$$


here we write $X^{t t}=X$ meaning we have a canonical, functorial isomorphism, which is denoted by

$$
\kappa_{X}: X \stackrel{\sim}{\longrightarrow} X^{t t}
$$

for abelian schemes, and for $p$-divisible groups.

(Biext) Let $N \rightarrow S$ be a finite flat group scheme. By [54], 1.1.4 and 3.3.2, see page 258 , we know that

$$
\operatorname{Ext}\left(N_{1}, N_{2}^{D}\right) \stackrel{\sim}{\longrightarrow} \operatorname{Biext}^{1}\left(N_{1}, N_{2} ; \mathbb{G}_{m}\right) .
$$

We write $\epsilon \mapsto E$ for the extension and the biextension corresponding under this canonical identification. If $(X, \lambda)$ is as above, and $q$ is a positive integer, $N:=X[q]$, we write $\left.(X, \lambda)\right|_{N}$ for the restriction of the biextension defined by $(X, \lambda)$

$$
\left.\right|_{N}: \operatorname{Biext}^{1}\left(X_{1}, X_{2} ; \mathbb{G}_{m}\right) \longrightarrow \operatorname{Biext}^{1}\left(N_{1}, N_{2} ; \mathbb{G}_{m}\right) .
$$

Suppose $P$ and $Q$ are $\mathrm{BT}_{1}$. We say that

$$
\text { ( } \epsilon) \quad 0 \rightarrow Q \rightarrow T \rightarrow P \rightarrow 0
$$

is a 2-extension if $Q=T[p]$ and $[p]: T \rightarrow \operatorname{Im}([p]: T \rightarrow T)=T / T[p]=P$ under the natural identifications. We say that $\epsilon$ is anti-symmetric if $\epsilon^{D D}=-\epsilon$; we intend to say: the exact sequence $\epsilon$ defines

$$
\left(\epsilon^{D}\right) \quad 0 \rightarrow P^{D} \rightarrow T^{D} \rightarrow Q^{D} \rightarrow 0 ;
$$

dualizing again we obtain $\epsilon^{D D}$; for finite flat group schemes we have a duality isomorphism

$$
\iota_{P}: P \stackrel{\sim}{\longrightarrow} P^{D D}
$$

(denoted by $\kappa$ in [42], I.2-2, but we use a different symbol because we have used already $\kappa$ for abelian schemes); we intend to say:

$$
\left(\iota_{P}\right)^{*}\left(\epsilon^{D D}\right)=-\left(\iota_{Q}\right) *(\epsilon) .
$$

We write $\operatorname{Ext}\left(N_{1}, N_{2}^{D} ; \mathbb{G}_{m}\right)^{(2, a)}$ for the set of 2-extension which are antisymmetric.

(Pairings) Let $K \supset \mathbb{F}_{p}$ be a perfect field, and let $N$ be a finite group scheme over $K$. We consider $\langle,>: A \times A \rightarrow W$, a pairing on the Dieudonné module $A:=\mathbb{D}(N)$. If $(X, \lambda)$ is as above, and $N:=X[p]$, we write $(X, \lambda)[p]=(N,<,>)$ for the pairing induced by $\lambda$ on $A=\mathbb{D}(A)$.

Note the different notations. If $(X, \lambda)$ is a polarized abelian scheme, and $N:=$ $X[p]$, we write $(N, E)=\left.(X, \lambda)\right|_{N}$ for the pair obtained by the restriction of 
the biextension, and we write $(X, \lambda)[p]=(N,<,>)$ for the pair obtained by restricting the polarization morphism to $N$.

Between these concepts there are some relations. Most of these are well-known, and we list them for convenience.

(12.2) Lemma (1) Let $(X, \lambda)$ be a polarized abelian scheme. Let $q$ be a positive integer, and $N:=\mathcal{X}[q]$. The restriction of $\lambda \in \operatorname{Biext}^{1}\left(X_{1}, X_{2} ; \mathbb{G}_{m}\right)$ gives a symmetric biextension $E \in \operatorname{Biext}\left(N_{1}, N_{2} ; \mathbb{G}_{m}\right)$, corresponding with an antisymmetric extension $\epsilon \in \operatorname{Ext}^{1}\left(N_{1}, N_{2}^{D}\right)$.

(2) If moreover $q=p$, and the polarization $\lambda$ is principal, the related extension $\epsilon$ is an anti-symmetric 2-extension:

$$
\left.\lambda\right|_{N}=\epsilon \in \operatorname{Ext}\left(N_{1}, N_{2}^{D} ; \mathbb{G}_{m}\right)^{(2, a)} .
$$

(3) Let $K \supset \mathbb{F}_{p}$ be a perfect field, and $N:=X[p]$. A principal polarization restricts to a non-degenerate pairing $(X, \lambda)[p]=(N,<,>)$ on $A:=\mathbb{D}(N)$ which is alternating.

(4) An anti-symmetric 2-extension on a $B T_{1}$ over a perfect field restricts to a non-degenerate, alternating pairing $(N,<,>)$ on $A:=\mathbb{D}(N)$.

(5) Let $k \supset \mathbb{F}_{p}$ be an algebraically closed field. The restriction map from $\operatorname{Ext}\left(N_{1}, N_{2}^{D} ; \mathbb{G}_{m}\right)^{(2, a)}$ to the set of pairs $(N,<,>)$ of $B T_{1}$ group schemes with a non-degenerate alternating pairing on its Dieudonné module is surjective. [Remark: in general this surjection is far from being injective.]

Proof. (1). This follows basically from [40], Coroll. (1.3.), pp. 69/70. In fact, if $\psi: X \rightarrow Y$ is an isogeny of abelian schemes, the duality theorem gives a canonical identification

$$
\nu: \operatorname{Ker}\left(\psi^{t}\right) \stackrel{\sim}{\longrightarrow}(\operatorname{Ker}(\psi))^{D} .
$$

The last diagram in the corollary cited reads: $-\nu \cdot \iota_{N}=\nu \cdot\left(\left.\kappa_{X}\right|_{N}\right)$. This proves (1).

(2). The extension is obtained as $\epsilon=\left(N_{1} \rightarrow N_{2}^{D}\right)^{*}\left(\left(\mathcal{X}^{t}\left[p^{2}\right] / \mathcal{X}^{t}[p]\right) \cong \mathcal{X}^{t}[p]\right)$. Hence it is a 2 -extension. This proves (2).

(3) and (4). The pairing on $A:=\mathbb{D}(N)$, where $N=X[p]$ in (3) can be defined as follows: consider a 2-extension

$$
0 \rightarrow N_{2}^{D} \longrightarrow T \longrightarrow N_{1} \rightarrow 0
$$

Then multiplication by $p$ on each member of this exact sequence, and applying the snake lemma we obtain an isomorphism $N_{1} \rightarrow N_{2}^{D}$; this is the pairing we are looking for. Let us make it explicit in terms of Dieudonné modules. Let $B:=\mathbb{D}(T)$. This is a free module over $W_{2}=W_{\infty}(k) /\left(p^{2}\right)$. The isomorphism $T \rightarrow T^{D}$, anti-symmetric by $(2)$, is written out by a non-degenerate, antisymmetric pairing [,] $B \times B \rightarrow W_{2}$, and the pairing $<,>$ on $A$ obtained form 
the extension can be described as: for $x \in A_{1}, y \in A_{2}$, we choose $x^{\prime} \in B$ such that $x^{\prime} \bmod A_{2}^{D}=x$; note that $A_{2}^{D} \subset B$; with these notations:

$$
<x, y>=\left(p x^{\prime}\right)(y),
$$

and using $B \cong B^{D}$ by [,] and $\left.\left(A_{2}^{D} \rightarrow B\right)^{D}=B^{B} \rightarrow A_{2}\right)$ we can rewrite this as

$$
<x, y>=p \cdot\left[x^{\prime}, y^{\prime}\right]
$$

We remark that $-1 \neq+1 \in W_{2}$. As the pairing [,] is anti-symmetric this implies that for every $z \in B$, with $z \notin p B$ we have $[z, z]=-[z, z]$, hence $p \cdot[z, z]=0$. We obtain $\langle x, x\rangle=p \cdot\left[x^{\prime}, x^{\prime}\right]=0$ for every $x \in A$. This proves $(3)$ and (4).

Remark. We do not have (in case the characteristic of the base field equals two) a characterization of those pairings on $N=\mathcal{X}[p]$ which come from a principal polarization on $\mathcal{X}$ only in terms of group schemes, not using $\mathbb{D}(N)$.

(12.3) Construction. Given a pair $(N,<,>)$ of a $B T_{1}$ with a nondegenerate alternating pairing on $A=\mathbb{D}(N)$ over $k$, we construct a $p$-divisible group $G$, and a principal quasi-polarization $\lambda$ on $G$ such that the restriction $\left(G, \lambda: G \rightarrow G^{t}\right)[p]$ gives $\left(N, \zeta: N=G[p] \rightarrow N^{D}=G^{t}[p]\right)$. - From this it clearly follows that the restriction map in (5) is surjective. One could indicate the polarized $p$-divisible group obtained below by something like Can $(\varphi)$. Note that in general $\operatorname{Can}(\operatorname{ES}(G, \lambda)) \approx(G, \lambda)$.

Indeed, we use the structure theorem of standard types as in Section 9. Hence we can choose a basis $\left\{X_{1}, \cdots, X_{g}, Y_{1}, \cdots, Y_{g}\right\}$ with the properties specified above. We choose $M:=\bigoplus W \cdot X_{i}^{\prime} \oplus \bigoplus W \cdot Y_{j}^{\prime}$, and we construct the structure of a Dieudonné module on this. We analyze the construction (9.1). We denote the base vectors in the standard type describing $A=\mathbb{D}(N)$ by $\left\{Z_{1}, \cdots, Z_{2 g}\right\}=\left\{X_{1}, \cdots, Y_{g}\right\}$. We have seen that $\left\{Z_{1}, \cdots, Z_{g}\right\}=$ $\left\{X_{1}, \cdots, X_{g-a}, Y_{g}, \cdots, Y_{g-a+1}\right\}$ is a $k$-base for $\mathcal{F}(A)=A[\mathcal{V}]$, and every of these base vectors is an image under $\mathcal{F}$ of one of the base vectors, in fact $\mathcal{F}\left(X_{i}\right)=Z_{i}$. We have also seen that each of the elements of $\left\{Z_{g}, \cdots, Z_{2 g}\right\}=$ $\left\{Y_{g-a}, \cdots, Y_{1}, X_{g-a+1}, \cdots, X_{g}\right\}$ is mapped under $\mathcal{V}$ onto either $-Y_{i}$ or onto $+Y_{i}$. For every $1 \leq i \leq g$ the image $\mathcal{F}\left(X_{i}\right)=Z_{j} \in A=M / p M$ is defined, and we require $\mathcal{F}\left(X_{i}^{\prime}\right)=\bar{Z}_{j}^{\prime} \in M$. For every $g<i \leq 2 g$ the image $\mathcal{V}\left(Z_{i}\right)=Z_{j}$ is defined, and we require $\mathcal{V}\left(Z_{i}^{\prime}\right)=Z_{j}^{\prime}$.

If $1 \leq i \leq a$ and $X_{i}=\mathcal{F}\left(X_{j}\right)$, we require $\mathcal{V}\left(X_{i}^{\prime}\right)=p \cdot X_{j}^{\prime}$.

If $g \geq i>g-a$ and $Y_{i}=\mathcal{F}\left(X_{j}\right)$ we require $\mathcal{V}\left(Y_{i}^{\prime}\right)=p \cdot X_{j}^{\prime}$.

In this way we have defined $\mathcal{V}$ for all base vectors $Z_{i}^{\prime}$.

If $a \geq j \geq 1$ and $Y_{i}=\mathcal{V}\left(Y_{j}\right)$ we require $\mathcal{F}\left(Y_{i}^{\prime}\right)=p \cdot Y_{j}^{\prime}$.

If $a \geq j \geq 1$ and $-Y_{i}=\mathcal{V}\left(X_{j}\right)$ we require $\mathcal{F}\left(Y_{i}^{\prime}\right)=-p \cdot X_{j}^{\prime}$.

In this way we have defined the image under $\mathcal{F}$ and under $\mathcal{V}$ of every base 
vector (and every image is either \pm a base vector, or $p$ times \pm a base vector). We extend the map $\mathcal{F}$ in a $\sigma$-linear way and $\mathcal{V}$ in a $\tau$-linear way on $\mathrm{M}$, where $\tau=\sigma^{-1}$. Direct verification shows that this defines the structure of a Dieudonné module of a $p$-divisible group with a symplectic $W$-base $\left\{Z_{i}^{\prime} \mid 1 \leq i \leq 2 g\right\}$. By construction, modulo $p$, this gives back the standard type which is $k$-isomorphic with $A$ with its symplectic form; this ends the construction.

Hence the proof of the lemma is complete.

(12.4) Lemma. Let $K$ be a perfect field. Let $\left(N_{1}, \zeta_{1}\right)$ and $\left(N_{2}, \zeta_{2}\right)$ be finite group schemes with an anti-symmetric symmetry over $K$. Let $A_{i}:=\mathbb{D}\left(N_{i}\right)$ be their Dieudonné modules. Let $f: A_{1} \rightarrow A_{2}$ be a $W$-linear, bijective map, where $W=W_{\infty}(K)$. Suppose that $f$ commutes with Frobenius and with the symmetries, i.e. the diagrams

$$
\begin{array}{lcccccc}
A_{1}^{(p)} & \stackrel{f(p)}{\rightarrow} & A_{2}^{(p)} & & A_{1} & \stackrel{\zeta_{1}}{\rightarrow} & A_{1}^{D} \\
\mathcal{F} \downarrow & & \downarrow \mathcal{F} & \text { and } & f \downarrow & & \uparrow f^{D} \\
& & & & & & \\
A_{1} & \stackrel{f}{\rightarrow} & A_{2} & & A_{2} & \stackrel{\zeta_{2}}{\longrightarrow} & A_{2}^{D}
\end{array}
$$

are commutative. Then $f$ is an isomorphism of Dieudonné modules, hence

$$
\left(N_{1}, \zeta_{1}\right) \stackrel{\sim}{\longrightarrow}\left(N_{2}, \zeta_{2}\right)
$$

Proof. On $A_{1}$ and on $A_{2}$ we have the Verschiebung, $\mathcal{V}: A_{i} \rightarrow A_{i}^{(p)}$. We have to show that $f$ also commutes with these maps in order to conclude that $f$ is an an isomorphism of Dieudonné modules. We show this by pushing $\mathcal{F}$ with the help of the $\zeta_{i}$ onto the duals, and then by duality transforming $\mathcal{F}$ on the duals into $\mathcal{V}$ on the $A_{i}$.

At first we show:

$$
\left(A_{2}^{(p) D} \rightarrow A_{2}^{D} \rightarrow A_{1}^{D}\right)=f^{D} \cdot \mathcal{F} \stackrel{?}{=} \mathcal{F} \cdot f^{(p) D}=\left(A_{2}^{(p) D} \rightarrow A_{1}^{(p) D} \rightarrow A_{1}^{D}\right)
$$

We should draw a large diagram, and chasing leads to:

$$
\begin{aligned}
&\left(A_{1}^{(p)} \rightarrow\right.\left.A_{2} \rightarrow A_{2}^{(p) D} \rightarrow A_{2}^{D} \rightarrow A_{1}^{D}\right)=\left(f^{D} \cdot \mathcal{F} \cdot\left(\zeta_{2}^{(p)} \cdot f^{(p)}\right)\right)= \\
&=-f^{D} \cdot \mathcal{F} \cdot \zeta_{2}^{(p) D D} \cdot \kappa \cdot f^{(p)}=f^{D} \cdot \zeta_{2} \cdot(f \cdot \mathcal{F})= \\
& f^{D} \cdot \zeta_{2} \cdot(f \cdot \mathcal{F})=\zeta_{1} \cdot \mathcal{F}=\mathcal{F} \cdot f^{D D} \cdot\left(\zeta_{2}^{(p)} \cdot f^{(p)}\right) ;
\end{aligned}
$$

hence

$$
\left(f^{D} \cdot \mathcal{F}\right)=\left(\mathcal{F} \cdot f^{(p) D}\right): A_{2}^{(p) D} \rightarrow A_{1}^{D}
$$

We use:

$$
\left(A_{i}^{(p) D} \stackrel{\mathcal{F}}{\longrightarrow} A_{i}^{D}\right)^{D}=\left(A_{i}^{(p) D}=A_{i}^{(p)} \stackrel{\mathcal{V}}{\longleftarrow} A_{i}=A_{i}^{D D}\right)
$$


under the identification $\iota: A_{i} \rightarrow A_{i}^{D D}$. This proves that $f$ "commutes" with $\mathcal{V}$, and the lemma is proved.

We show that the boundary of a stratum is a union of (lower dimensional) strata:

(12.5) Proposition. Let $\varphi \in \Phi$ be an elementary sequence, let $\left[\left(X_{0}, \lambda_{0}\right)\right]=$ $0 \in\left(S_{\varphi}\right)^{c}$, and let $\left[\left(Y_{s}, \mu_{s}\right)\right]=s \in \mathcal{A}$ such that $\operatorname{ES}\left(X_{0}, \lambda_{0}\right)=\operatorname{ES}\left(Y_{s}, \mu_{s}\right)$. Then $s \in\left(S_{\varphi}\right)^{c}$. This implies:

$$
S_{\varphi^{\prime}} \cap\left(\left(S_{\varphi}\right)^{c}\right) \neq \emptyset \quad \Longrightarrow \quad S_{\varphi^{\prime}} \subset\left(S_{\varphi}\right)^{c} .
$$

Proof. It suffices to show this under the extra condition that $X_{0}[p]$ is a locallocal group scheme, i.e. $\varphi^{\prime}(1)=0$. We suppose $k$ is an algebraically closed field, with $\left(X_{0}, \lambda_{0}\right) \in \mathcal{A}(k)$, over which $\left(Y_{s}, \mu_{s}\right)$ is also defined. As $0 \in\left(S_{\varphi}\right)^{c}$ we can choose a complete local domain $R$ of characteristic $p$, with residue class field $k$, and an abelian scheme $(X, \lambda) \rightarrow \Delta=\operatorname{Spec}(R)$ such that $(X, \lambda) \otimes_{R} k=\left(X_{0}, \lambda_{0}\right)$ and for the generic point $\eta \in \Delta$ we have $\operatorname{ES}\left(X_{\eta}, \lambda_{\eta}\right)=\varphi$. As $\operatorname{ES}\left(X_{0}, \lambda_{0}\right)=$ $\varphi^{\prime}=\operatorname{ES}\left(Y_{s}, \mu_{s}\right)$, using (9.4), (!), we can choose an isomorphism (which we will write as an identification):

$$
\left(X_{0}, \lambda_{0}\right)[p]=\left(N_{0}, \zeta_{0}\right)=\left(Y_{s}, \mu_{s}\right)[p] .
$$

Let $\left\{Z_{i}\right\}$ be a symplectic $W$-base for $M_{0}:=\mathbb{D}\left(X_{0}\left[p^{\infty}\right]\right)$ such that $Z_{g+1}, \cdots, Z_{2 g} \in \mathcal{V} M$. On this basis the matrix of $\mathcal{F}$ on $M_{0}$ is given by the display

$$
\left(\begin{array}{cc}
A & B \\
C & D
\end{array}\right) \text { and }\left(\begin{array}{cc}
A+T C & B+T D \\
C & D
\end{array}\right)
$$

is the display of the deformation $\left(X_{0}, \lambda_{0}\right) \subset(X, \lambda)$, where $T \in \operatorname{Mat}(g \times$ $\left.g, W_{\infty}(R)\right)$ is a symmetric matrix. We choose a symplectic basis for $\mathbb{D}\left(Y_{s}\left[p^{\infty}\right]\right)$ such that this modulo $p$ coincides with $\left\{Z_{i} \bmod p\right\}$ on $\mathbb{D}\left(X_{0}[p]\right)=\mathbb{D}\left(N_{0}\right)=$ $\mathbb{D}\left(Y_{s}[p]\right)$. We write

$$
\left(\begin{array}{ll}
a & b \\
c & d
\end{array}\right)
$$

for the display on this basis on $\mathbb{D}\left(Y_{s}\left[p^{\infty}\right]\right)$. Note that

$$
\left(\begin{array}{ll}
A \bmod p & 0 \\
C \bmod p & 0
\end{array}\right)=\left(\begin{array}{cc}
a \bmod p & 0 \\
c \bmod p & 0
\end{array}\right) .
$$

We define a deformation of $\left(Y_{s}\left[p^{\infty}\right], \mu_{s}\right)$, and hence by the theorem of Serre and Tate, a deformation $(Y, \mu)$ of $\left(Y_{s}, \mu_{s}\right)$, by the display

$$
\left(\begin{array}{cc}
a+T c & b+T d \\
c & d
\end{array}\right)
$$


We claim: $\operatorname{ES}\left(Y_{\eta}, \mu_{\eta}\right)=\varphi$. Indeed, let $K$ be a perfect field containing $Q(R)$. We see that $\mathcal{F}$ on $\mathbb{D}\left(X_{\eta}\right)[p] \otimes K$ is given by the same display

$$
\left(\begin{array}{cc}
(A+T C) \bmod p & 0 \\
C \bmod p & 0
\end{array}\right)=\left(\begin{array}{cc}
(a+T c) \bmod p & 0 \\
c \bmod p & 0
\end{array}\right)
$$

as the one giving $\mathcal{F}$ on $\mathbb{D}\left(Y_{\eta}\right)[p] \otimes K$, and the pairings on these finite group schemes coincide under this identification. Hence the conditions of the previous lemma are fulfilled, and we conclude that $\left(X_{\eta}, \lambda\right)[p] \otimes K \cong\left(Y_{\eta}, \mu\right)[p] \otimes K$. Hence $\operatorname{ES}\left(X_{\eta}, \lambda_{\eta}\right)=\operatorname{ES}\left(Y_{\eta}, \mu_{\eta}\right)=\varphi$, and the proof of the proposition is concluded.

We expect that a proof of the previous proposition can be given by local deformation theory, by using [17], and giving an interpretation of the image of biextension on finite group schemes to a certain set of anti-symmetric pairings over arbitrary base schemes. This was our original approach, which works in characteristic $p>2$, also see [61]. We have not carried out this programme for $p=2$.

(12.6) Remark. It seems plausible (using the notations in the previous proof) that $(X, \lambda)[p] \cong(Y, \mu)[p] \rightarrow \Delta$. However, in general there is no $(Y, \mu) \rightarrow \Delta$ extending $\left(Y_{s}, \mu_{s}\right)$ such that $\left.(X, \lambda)\right|_{N}$ and $\left.(Y, \mu)\right|_{N}$ are isomorphic over $\Delta$, where $X[p]=N=Y[p]$; in other terms: the finite group scheme with the symmetry $(N, \zeta)$ can be included in a new $(Y, \mu) \supset\left(Y_{s}, \mu_{s}\right)$, but in general the finite group scheme with biextension $(N, E)$ does not admit such a construction.

\section{Proof of the results}

The set of superspecial points $\Sigma \subset \mathcal{A}$ is a finite set of points. It is clear that

$$
\mathrm{ES}(X)=g \quad \Longleftrightarrow \quad a(X)=g \quad \Longleftrightarrow \quad X \quad \text { is superspecial, }
$$

i.e. there is a supersingular elliptic curve $E$ and an isomorphism $X \otimes k \cong E^{g}$.

(13.1) In Section 7 we have seen the construction of $L$, in (7.3) we have proved that $L$ is connected. In Section 8 we have proved that $L=\left(S_{\{0, \cdots, 0,1\}}\right)^{c}$. $\square$ Theorem (1.1)

(13.2) In Section 11 we have seen how to produce new abelian varieties with an elementary sequence $\varphi^{\prime}$ (see the notation in that section). Hence it follows by induction from the superspecial locus that every elementary sequence does appear, i.e. for every $\varphi \in \Phi$ the locus $S_{\varphi}$ is non-empty. Using Section 10 and Section 11, in particular (11.2) it follows that $\operatorname{dim}\left(S_{\varphi}\right)=|\varphi|$.

$\square$ Theorem (1.2). 
(13.3) In (12.5) we have proved that a locus $S_{\varphi^{\prime}}$ either does not meet $\left(S_{\varphi}\right)^{c}$ or is contained in $\left(S_{\varphi}\right)^{c}$ : this is the first claim in Theorem (1.3).

Let $\varphi \in \Phi$, with $\varphi \neq\{0, \cdots, 0\}$, i.e. $S_{\varphi} \neq \Sigma$. By (6.5) we know that every irreducible component $T \subset T_{\varphi} \subset \mathcal{A}^{*}$ is quasi-affine; because its dimension is positive, this implies that its boundary $\partial_{\mathcal{A}^{*}}(T) \neq \emptyset$. By $(6.3)$ we conclude that $\partial_{\mathcal{A} *}(T) \cap \mathcal{A}$ is non-empty; hence we see that every irreducible component $S \subset S_{\varphi}$ is not complete. Moreover using (12.5) this proves that every irreducible component $S \subset S_{\varphi}$ has a non-empty boundary in $\mathcal{A}$, which is of codimension one by (4.1), and we see it is a union of components of lower dimensional strata. Hence by induction we see that every component of $S_{\varphi}$ contains in its boundary a component of the (unique) zero-dimensional stratum $S_{\{0, \cdots, 0,1\}}$; hence by $(8.4)$ we know that $\partial(S)$ contains an irreducible component of $L$. Hence if $\varphi \neq\{0, \cdots, 0\}$, i.e. $S_{\varphi} \neq \Sigma$, then $\left(S_{\varphi}\right)^{c}$ is connected.

$\square$ Theorem (1.3)

Remark. Instead of using (6.5) in the proof of (1.3), we can use (6.3), induction on $g$, and (12.5).

As $\mathcal{A}=\left(S_{\{1,2, \cdots, g\}}\right)^{c}$, we know by Theorem (1.3) that it is connected. Moreover for $n \geq 3$ it is non-singular. Hence $\mathcal{A}_{g, 1, n} \otimes \mathbb{F}_{p, n}$ is geometrically irreducible for every $g$ and every $n$ (not divisible by $p$ ).

$\square$ Corollary (1.4)

(13.4) It suffices to show (1.5) in case we moreover assume $n>3$. We note that the locus $V_{g-1} \subset \mathcal{A}$ of non-ordinary polarized abelian varieties equals $V_{g-1}=\left(S_{\{0,1,2, \cdots, g-1\}}\right)^{c}$. Let us work over an algebraically closed field $k$. Every component of this closed locus contains at least one irreducible component of $L$, and it contains at least one point of $\Sigma$.

Let $\left[\left(X_{0}=E^{g}, \lambda\right)\right]=x \in \Sigma \subset V_{g-1}$. By [29], 6.1 we can put a principal polarization on $E^{g}$ in diagonal form. We write out the display of the universal deformation of $\left(X_{0}=E^{g}, \lambda\right)$ on this basis. The Hasse-Witt matrix of $X_{0}$ is zero, and we see that a formal neighborhood of $x$ in $\mathcal{A}$ is given by $\operatorname{Spf}\left(k\left[\left[T_{i, j} \mid 1 \leq i, j \leq g\right]\right] /\left(T_{i, j}-T_{j, i}\right)\right)$; moreover

$$
V_{g-1} \otimes k \text { at } x \text { is given by } \operatorname{Det}\left(T_{i, j}\right)=0 .
$$

Claim. This equation is given by a non-zero, irreducible polynomial. This we prove by induction on $g$. For $g=1$ this is clear. Let $T=T_{g, g}$, and write

$$
\operatorname{Det}\left(T_{i, j} \mid 1 \leq i, j \leq g\right)=: D_{g}=A \cdot T+B,
$$

where $A=D_{g-1}$ and $B$ are polynomials in the other variables. The polynomial $A$ is non-zero, and for $g>1$ it is irreducible by the induction assumption. For $g>1$ we substitute $T_{j, j}=1$ for all $j<g-1$, and $T_{g-1, g}=T_{g, g-1}=1$, and all other variables $=0$; this gives $B \mapsto 1$ and $A \mapsto 0$; because $A$ is irreducible we see that $A$ and $B$ are coprime. Hence $A T+B$ is irreducible. 
Thus we see that $V_{g-1} \otimes k$ is locally irreducible at all $x \in \Sigma$. Hence for every irreducible component $L^{\prime} \subset L_{k}$ there is exactly one component of $V_{g-1} \otimes k$ containing $L^{\prime}$. Hence there is precisely one component of $V_{g-1} \otimes k$ having nonempty intersection with $L_{k}$. By what has been said before this implies that $V_{g-1}$ is geometrically irreducible.

$\square$ Corollary (1.5)

\section{Some questions}

(14.1) We have seen that some strata are reducible (like $\Sigma$, like $L$, say for large $p$ ). Some strata are irreducible (like $\mathcal{A}$, like $V_{1}$ for $g>1$ ). What can be said in general? We expect:

Conjecture. Let $\varphi \in \Phi$ such that $S_{\varphi}$ is not contained in the supersingular locus. Then we expect that $\left(S_{\varphi}\right)^{c}$ is geometrically irreducible.

This is in agreement with the examples just mentioned; it was proved in certain cases by Van der Geer, see [11], Theorem (9.9): take $\varphi=\{1,2, \cdots, g-$ $a, \cdots, g-a\}$, with $a<g$.

(14.2) In (4.1) we have seen that $T_{\varphi} \subset \mathcal{A}^{*}$ is a quasi-affine set, and the same for $S_{\varphi}$. We expect that $T_{\varphi}$ is affine subscheme of $\mathcal{A}^{*}$ for every $\varphi$. We expect that there exists $\varphi$ such that $S_{\varphi} \subset \mathcal{A}$ is not affine.

(14.3) Given two elementary sequences $\varphi, \varphi^{\prime} \in \Phi$ we have defined the relation $\varphi^{\prime} \prec \varphi$ by $\varphi^{\prime}(i) \leq \varphi(i)$ for all $i$. We define

$$
\varphi^{\prime} \subset \varphi \stackrel{\text { def }}{\Longleftrightarrow} S_{\varphi^{\prime}} \subset\left(S_{\varphi}\right)^{c}
$$

It follows from (11.1) that $\varphi^{\prime} \prec \varphi \Rightarrow \varphi^{\prime} \subset \varphi$. It is not so difficult to give examples that the converse does not hold:

Example. We choose $\varphi^{\prime}, \varphi \in \Phi_{7}$, and deformation which shows that

$$
\varphi^{\prime} \subset \varphi, \text { while } \varphi^{\prime} \nprec \varphi \text {. }
$$

We choose $\varphi^{\prime}=\{0,0,1,1,2,2,2\}$, hence $\psi^{\prime}=\{0,0,1,1,2,2,2 ; 3,4,4,5,5,6,7\}$; we choose $\varphi=\{0,0,0,1,2,3,3\}$, hence $\psi=\{0,0,0,1,2,3,3 ; 4,4,4,4,5,6,7\}$; clearly $\varphi^{\prime} \nprec \varphi$; we prove that $\varphi^{\prime} \subset \varphi$. The standard type for $\varphi^{\prime}$ has a Dieudonné module with basis:

$$
\left\{Y_{7}, X_{1}, Y_{6}, X_{2}, Y_{5}, Y_{4} ; X_{3}, X_{4}, Y_{2}, X_{5}, Y_{1}, X_{6}, X_{7}\right\}
$$

We write out a display, where $T_{4,5}=T=T_{5,4}$, and all other other variables are put to zero. Explicitly, on the basis given above, we have $\mathcal{F}: X_{6} \mapsto Y_{4}+T X_{4}=$ : $Z$, and all other images under $\mathcal{F}$ of base vectors are the same as before: under $\mathcal{F}$, all $Y_{i}$ are mapped to zero, and we have $X_{7} \mapsto Y_{3}$ and $X_{6} \mapsto Z \mapsto T^{\sigma} \cdot Y_{5}$, and 
$X_{5} \mapsto X_{2} \mapsto Y_{6}$ and $X_{4} \mapsto Y_{5}$ and $X_{3} \mapsto X_{1} \mapsto Y_{7}$. Over the field of fractions $k((T))$ we choose a basis by:

$\left\{Y_{7}, Y_{6}, Y_{5}, X_{1}, X_{2}, \xi_{3}=Z=Y_{4}+T X_{4}, \eta_{4}=Y_{3} ; \xi_{4}=X_{3}, \eta_{3}=(1 / T) Y_{4}, Y_{2}, Y_{1}, X_{5}, X_{6}, X_{7}\right\}$;

this puts the generic fiber of the deformed module in standard form, with elementary sequence $\varphi$ ).

We note that $\varphi^{\prime}$ is canonical, and it belongs to the cycles $\{\mathcal{F V} \mathcal{V} ; \mathcal{V} \mathcal{F} \mathcal{F} ; \mathcal{F} \mathcal{V} \mathcal{F} \mathcal{V} \mathcal{F} \mathcal{V} \mathcal{V}\}$; the final sequence belonging to $\varphi$ is not canonical; it belongs to the cycles $\left\{\mathcal{F V V} ; \mathcal{V} \mathcal{F} \mathcal{F} ;(\mathcal{F F V V})^{2}\right\}$; we have seen that $\varphi^{\prime} \subset \varphi$. However, consider the cycles $\{\mathcal{F} \mathcal{V} \mathcal{F} \mathcal{F} \mathcal{F} \mathcal{V} \mathcal{V}\}$ and $\left\{(\mathcal{F F} \mathcal{V} \mathcal{V})^{2}\right\}$; these define $\beta^{\prime}, \beta \in \Phi_{4}$, and they correspond with elementary sequences $\{0,1,1,1\}$ and $\{0,0,1,2\}$; hence, although $\varphi^{\prime} \subset \varphi$, "subtracting equal parts", gives $\beta^{\prime} \subsetneq \beta$ and $\beta^{\prime} \supsetneq \beta ! ?$

We see: in general

$$
\varphi \subset \varphi^{\prime} \nRightarrow \varphi \prec \varphi^{\prime}
$$

Question. Is there an easy algorithm, using the combinatorics of sequences, describing the relation $\varphi \subset \varphi^{\prime}$ ?

(14.4) On $\mathcal{A}$ we have defined a stratification $\mathcal{A}=\sqcup_{\varphi \in \Phi} S_{\varphi}$. For every symmetric Newton polygon $\beta$ there is a stratum $W_{\beta} \subset \mathcal{A}$; this is the (closed) set of points where the Newton polygon is either equal or above $\beta$; we write $W_{\beta}^{0}$ for the locally closed set of points where the Newton polygon is equal to $\beta$; we have $\mathcal{A}=\sqcup_{\beta} W_{\beta}^{0}$; for this structure, see [47]. Both stratifications are reasonably well understood by now. However intersections are not so easy to understand. For example, I have some information, but no proven complete results, which tell us exactly which elementary sequences appear on the set $W_{\beta}^{0}$.

(14.5) Even more difficult is the question to describe intersections of all NPstrata, and of all EO-strata with the Torelli locus $j\left(\mathcal{M}_{g}\right)=\mathcal{T}_{g}^{0} \subset\left(\mathcal{T}_{g}^{0}\right)^{c}=: \mathcal{T}_{g} \subset$ $\mathcal{A}$; in these case I have no reasonable conjecture to offer. Here is an example: Question. Does every symmetric Newton polygon appear on $\mathcal{T}_{g}$ ?

It is known that even for large values of $g$ there are supersingular curves of genus $g$, contrary what might be thought after dimension considerations, see [12]. I expect the answer to this question to be negative for large $g$, but I have no reasonable theory, no method, no examples to support this expectation.

I do not know which elementary sequences appear on $\mathcal{T}_{g}$.

Also I have no idea to describe the intersection of $\mathcal{T}_{g}$ with either of the two foliations of $W_{\beta}^{0}$ which will be described in [48]. 
(14.6) Remark. Suppose that the answer to the question in (14.5) is negative, i.e. suppose there exists a symmetric Newton polygon which does not appear on the corresponding $\mathcal{T}_{g}$. Then it follows that there exists an abelian variety over $\overline{\mathbb{Q}}$ which is not isogenous with a Jacobian; this would answer a question asked by N. Katz (we expect a negative answer):

Question. Is every abelian variety over $\overline{\mathbb{Q}}$, or over $\overline{\mathbb{F} p}$ isogenous with a Jacobian? Note that over $\mathbb{C}$ there exists for every $g \geq 4$ an abelian variety not isogenous with a Jacobian.

(14.7) In general a p-divisible group $G$ is not determined by the structure of its p-kernel $G[p]$. For example, Choose $g=3$, and consider all principally polarized abelian varieties $(X, \lambda)$ with $f(X)=0$ and $a(X)=1$. We have seen that in such a case we have $(X[p],<,>) \cong N_{\{0,1,2\}}$, in the notation of Section 9 ; but we know that the formal isogeny type of $X\left[p^{\infty}\right]$ can be either $G_{2,1} \oplus G_{1,2}$ or $3 \cdot G_{1,1}$. Moreover, even if the formal isogeny type is given, then within one of these classes there are infinitely many mutually non-isomorphic possibilities. Many more examples along these line can be given.

However there are cases in which the $p$-kernel does determine the $p$ divisible group. For example, if $f(X)$ is maximal (the ordinary case), or if $a(X)$ is maximal (the superspecial case): over an algebraically closed field there is a unique isomorphism class for the $p$-divisible group having this $p$-kernel.

Also, in (8.3), case (I), we have seen other cases where the $p$-kernel determines the $p$-divisible group.

It would be nice to have a general criterion which tells us when it should be true that the $p$-kernel of a $p$-divisible group already determines the structure of the $p$-divisible group. To that end we formulate a conjecture, which includes the cases recorded above. We hope you can soon consult [48] for a more extensive discussion.

We work over an algebraically closed field $k$. Let $\beta=\sum\left(m_{i}, n_{i}\right)$ be a symmetric Newton polygon. We write $H(m, n)$ for the "minimal" $p$-divisible group in the isogeny class of $G_{m, n}$, see [19], (5.3), see (8.1) above. We write $H(\beta)=$ $\bigoplus H\left(M_{i}, n_{i}\right)$. There is an (obvious) principal quasi-polarization $\mu$ on $H(\beta)$ (note that $\beta$ is symmetric). For "minimal isogeny types" we could hope the following to be true:

(14.8) Conjecture. Suppose $(G, \lambda)$ is a p-divisible group with a principal quasi-polarization over an algebraically closed field $k$; suppose that there exists a symmetric Newton polygon $\beta$ and an isomorphism

$$
\left.\left.(G, \lambda)\right|_{G[p]} \cong(H(\beta), \mu)\right|_{H(\beta)[p]} ; \quad \text { then we expect: } \quad(G, \lambda) \cong(H(\beta), \mu)
$$


(14.9) Remark. We have seen that every elementary sequence appears in $\mathcal{A}_{g, 1} \otimes \mathbb{E}_{p}$. We have constructed for every symmetric canonical type $\tau$ a nonempty, locally closed subscheme $D_{\tau} \subset \mathcal{A}^{\prime}=\mathcal{A}_{g} \otimes \mathbb{F}_{p}$; however we do not know which finite group schemes $N=X[p]$ appear on a given irreducible component of $\mathcal{A}_{g} \otimes \mathbb{F}_{p}$, i.e. we do not know which strata $D_{\tau}$ for an arbitrary canonical type $\tau$ are non-empty, and how they appear in components of $\mathcal{A}^{\prime}$.

(14.10) The following is true: Let $S$ be a complete, irreducible scheme over $\mathbb{F}_{p}$. Let $G \rightarrow S$ be a p-divisible group, such that any two geometric fibers of $G[p] \rightarrow S$ are isomorphic; i.e. if $s, t \in S(k)$, with $k=\bar{k}$, then there exists an isomorphism $G[p]_{s} \cong G[p]_{t}$; equivalently: over some finite covering $T \rightarrow S$ the group scheme $G[p]_{T}$ is constant. Then it follows that any two geometric fibers of $G \rightarrow S$ are isomorphic.

We hope to publish a proof soon, and we expect to come back to this and related questions. Note that in general $G$ does not become constant over any finite cover. Note that in general the conclusion does not hold if we delete the condition "S is complete".

\section{Appendix. Some notations}

(15.1) Throughout this paper all base schemes, and all base fields, will be in characteristic $p$. Let $X$ be an abelian variety over a field $K$. The $p$-rank of $X$, denoted by $f=f(X)$ is defined by:

$$
X[p](k) \cong(\mathbb{Z} / p)^{f},
$$

where $k$ denotes the algebraic closed field containing $K$, and $X[p]$ is the group scheme $\operatorname{Ker}(\times p: X \rightarrow X)$. An abelian variety $X$ of dimension $g$ such that $f(X)=g$ (i.e. the case where the $p$-rank is maximal) is called ordinary.

We fix a positive integer $g$, a prime number $p$ and a positive integer $n$ not divisible by $p$, and we denote by

$$
\mathcal{A}=\mathcal{A}_{g, 1, n} \otimes \mathbb{F}_{p, n},
$$

the moduli space of principally polarized abelian varieties with a symplectic level- $n$-structure in characteristic $p$.

Let $G$ be a group scheme over a field $K$. We write $a(G):=\operatorname{dim}_{L} \operatorname{Hom}\left(\alpha_{p}, G_{L}\right)$, where $L \supset K$ is a perfect field containing $K$. Here $\alpha_{p}=\mathbb{G}_{a}[F]$, the kernel of the Frobenius morphism on the additive linear group of dimension one; this group scheme is defined over $\mathbb{F}_{p}$, and we will consider this over any base without further indicating over which base it is considered. An abelian variety is ordinary iff $a(X)=0$. 
Let $E$ be an elliptic curve over a field $K$ (of characteristic $p$ ). Then either $E$ has a point of order exactly $p$ over an algebraically closed field $k \supset K$, and in this case the elliptic curve $E$ is ordinary, or

$$
f(E)=0, \quad \text { and the curve is called supersingular. }
$$

In short: an elliptic curve is supersingular iff its $p$-rank is zero, iff $a(E)=1$. We say that an abelian variety $X$ is supersingular if it isogenous to a product of supersingular elliptic curves over some extension field. It is superspecial if it isomorphic to a product of supersingular elliptic curves over some extension field.

For a commutative group scheme $G$ and $q \in \mathbb{Z}_{>0}$ we denote by $G[q]$ the kernel of multiplication by $q$ on $G$. In this paper the $p$-kernel $X[p]$ of abelian varieties in consideration will play a central role. We write $X\left[p^{\infty}\right]$ for the union of the finite group schemes $X\left[p^{i}\right]$ for all $1 \leq i$; note that it contains the formal group $\hat{X}$ of $X$ and $\hat{X}=X\left[p^{\infty}\right]$ iff $f(X)=\overline{0}$.

(15.2) Here is a survey of some of the notions about supersingular and superspecial abelian varieties:

Theorem/Definition: Let $k$ be an algebraically closed field of characteristic $p$, and let $E$ be a supersingular elliptic curve over $k$. Let $X$ be an abelian variety of dimension $g$ over a field $K \subset k$. Let $g \geq 2$.

a)

$$
\hat{X}_{k} \sim \hat{E}^{g} \Longleftrightarrow X_{k} \sim E^{g} \stackrel{\text { def }}{\Longleftrightarrow} X \text { is supersingular. }
$$

b) If $E_{1}, \cdots, E_{2 g}$ are supersingular elliptic curves, then

$$
E_{1} \times \cdots \times E_{g} \cong E_{g+1} \times \cdots \times E_{2 g} .
$$

c) A supersingular elliptic curve is superspecial;

$$
\hat{X}_{k} \cong \hat{E}^{g} \Longleftrightarrow a(X)=\operatorname{dim} X \Longleftrightarrow X \cong E^{g} \stackrel{\text { def }}{\Longleftrightarrow} X \quad \text { is superspecial. }
$$

The first statement was proved in [43], Th. 4.2. Statement (b) is a theorem by Deligne [58], Th. 3.5 on page 580 , which uses a class number computation by Eichler, cf. [7]; also see [41]. For proving (c), note that if $\hat{X} \cong \hat{E}^{g}$, then $a(X)=\operatorname{dim} X$, and by [44] it follows that $X$ is isomorphic with a product of supersingular elliptic curves, $X \cong E_{1} \times \cdots \times E_{g}$; such a product is isomorphic with $E^{g}$ by (b). For further information, see [29].

(15.3) Covariant Dieudonné modules (with apologies to the Contravarianists.) Over a perfect field (of positive characteristic) finite group schemes 
and $p$-divisible groups can be classified by the theory of Dieudonné modules. This can be done in a contravariant way as in [40], [31]. One can also use the covariant theory. And it is not important which one we choose as long as we work over a perfect field: these two theories are anti-equivalent.

However as soon as we are working over a more general base ring (e.g. when studying deformation theory) things are different. For finite group schemes the theory is much more difficult, but see [17], [18]. For formal groups Cartier developed a theory of covariant Dieudonné modules which works well. Later this was developed and used in the disguise of displays, invented by Mumford, see [36], and see [38], [39], [63], [64]. Therefore, in all cases we shall work with the covariant theory.

For a domain $R$ of positive characteristic $p$ we write $W=W(R)=W_{\infty}(R)$ for the ring of infinite Witt vectors. The "Frobenius" $x \mapsto x^{p}$ on $R$ lifts to a ring homomorphism $\sigma: W \rightarrow W$. From now on we use a perfect field $K$. In that case $\sigma: W(K) \rightarrow W(K)$ is an isomorphism. We write $E$ for the ring "power series" in the variables $F$ and $V$ with coefficients in $W$ with the relations $F V=p=V F$ and $F a=a^{\sigma} F$ and $a V=V a^{\sigma}$ for $a \in W$. Note that $E$ is commutative iff $K=\mathbb{F}_{p}$.

Finite group schemes over a perfect field. Denote by $\mathcal{N}=\mathcal{N}_{\text {loc,loc, } K}$ the category of finite group schemes over the perfect field $K$ which are of local-local type. Dieudonné module theory tells us:

There is a covariant equivalence: $\mathbb{D}$ between $\mathcal{N}$ and the category of E-modules of finite length on which $F$ and $V$ act nilpotently. This equivalence has the following properties:

$$
\operatorname{rk}(N)=p^{t} \Longleftrightarrow \ell(\mathbb{D}(N))=t .
$$

Moreover (with apologies...):

$$
\begin{aligned}
& \mathbb{D}\left(F: N \rightarrow N^{(p)}\right)=\left(\mathcal{V}: A \rightarrow A^{(p)}\right), \\
& \mathbb{D}\left(V: N^{(p)} \rightarrow N\right)=\left(\mathcal{F}: A^{(p)} \rightarrow A\right) ;
\end{aligned}
$$

notation: the module $A^{(p)}$ is the one obtained from $A$ obtained by the base change $\sigma$. We have distinguished the action of Frobenius on group schemes, denoted by $F$, and the action on Dieudonné modules given by Frobenius, which we indicate by $\mathcal{F}$. In the central part of this paper, we consider an abelian variety $X$, we write $N:=X[p]$, we write $A:=\mathbb{D}(N)$ for the Dieudonné module of this $p$-kernel. We use the operation $V$ on $N$, which corresponds with $\mathcal{F}$ on A.

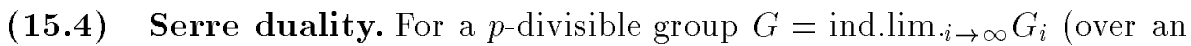
arbitrary base) we follow Serre in defining the dual $p$-divisible group:

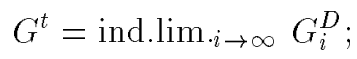


here $-^{D}$ is the Cartier dual, and $\times p: G_{i+1} \rightarrow G_{i}$ dualizes to inclusions $G_{i}^{D} \subset$ $G_{i+1}^{D}$ which we use to define $G^{t}$.

Note that the duality theorem on abelian schemes, see [42], Th. 19.1, implies that for an abelian scheme $X \rightarrow S$ we have:

$$
X\left[p^{\infty}\right]^{t}=X^{t}\left[p^{\infty}\right] .
$$

(15.5) Classification by Dieudonné and Manin, notation. In [31] we find a classification for isogeny classes of $p$-divisible groups over an algebraically closed field $k \supset \mathbb{E}_{p}$. For a pair of non-negative integers $m, n \in \mathbb{Z}_{>0}$ a $p$-divisible group $G_{n, m}$ is defined. We write $G_{1,0}=\mathbb{G}_{m}^{\wedge}=\mathbb{G}_{m}\left[p^{\infty}\right]$. We write $G_{0,1}$ for its dual, which in fact is the "constant group scheme" $\mathbb{Q}_{p} / \mathbb{Z}_{p}$.

For $m>0$ and $n>0$ which are relatively prime positive integers we write $G_{m, n}$ for a formal group of dimension $m$, whose Serre dual has dimension $n$, and which is "isosimple". It is defined by:

$$
\mathbb{D}\left(G_{m, n}\right)=W[[F, V]] / W[[F, V]] \cdot\left(F^{m}-V^{n}\right) .
$$

Over $\mathbb{F}_{p}$ already we have the isomorphism:

$$
\left(G_{m, n}\right)^{t}=G_{n, m} .
$$

The Dieudonné - Manin classification theorem reads:

Let $k \supset \mathbb{F}_{p}$ be an algebraically closed field. For a p-divisible group $G$ over $k$, of dimension $d$ and height $h$, there exist pairs $\left\{\left(m_{i}, n_{i}\right) \mid 1 \leq i \leq s\right\}$ and an isogeny:

$$
G \sim \sum_{i} G_{\left(m_{i}, n_{i}\right)}, \quad \sum m_{i}=d, \quad \sum\left(m_{i}+n_{i}\right)=h .
$$

The set of these pairs is called the formal isogeny type of $G$. In case $G \cong$ $X\left[p^{\infty}\right]$, the $p$-divisible group of an abelian variety the formal isogeny type is "symmetric" and we can change the notation above into:

$$
X\left[p^{\infty}\right] \sim f \cdot\left(G_{1,0} \oplus G_{0,1}\right) \bigoplus s \cdot G_{1,1} \bigoplus \sum_{i}^{<\infty}\left(G_{m_{i}, n_{i}} \oplus G_{n_{i}, m_{i}}\right),
$$

where $f \in \mathbb{Z}_{\geq 0}$, and $s \in \mathbb{Z}_{\geq 0}$, and the integers $m_{i}$, and $n_{i}$ are pairwise coprime with $m_{i}>n_{i}>0$.

A formal isogeny type can be encoded via the notion of a Newton polygon abbreviated NP: for a $p$-divisible group $G$ of height $h$ and dimension $d$ a Newton polygon is a polygon in $\mathbb{R}^{2}$ (or in $\mathbb{Q}^{2}$, if you prefer that), starting at $(0,0)$, ending at $(h, h-d)$, which is lower convex and which has break points in $\mathbb{Z}^{2}$. The $p$-divisible group $G_{m, n}$ gives the slope $n /(n+m)$ with multiplicity $n+m$. A direct sum as above gives a Newton polygon by ordering the slopes in increasing order. Note that the local-étale part gives all slopes equal to 0 , the local-local summands gives slopes strictly between 0 and 1 , and the étale-local part gives all slopes 1 . 
(15.6) Displays. This tool invented by Mumford, see [36], developed in [38], [39], and by Zink in various manuscripts, see [63], [64], [65]; this describes deformation theory by giving the Frobenius mapping on what we would like to consider as the "Dieudonné module of the deformed $p$-divisible group". In this paper we do not use the full strength of that method. What we need is the following (for more details on the notations, see [46], 1.9-1.12). Suppose $G_{0}$ is a $p$-divisible group over a perfect field $K \supset \mathbb{F}_{p}$, and let $M_{0}=\mathbb{D}\left(G_{0}\right)$ be its covariant Dieudonné module. Let $\left\{X_{1}^{\prime}, \cdots, X_{d}^{\prime}, Y_{1}^{\prime}, \cdots Y_{c}^{\prime}\right\}$ be a $W$-basis for $M_{0}$, such that $Y_{1}^{\prime}, \cdots Y_{c}^{\prime} \in \mathcal{V}(M)$; here we write $X_{i}^{\prime}$ etc. because in Section 9 the notation $X_{i}$, and $Y_{j}$ has been used for elements of a standard base for $\mathbb{D}\left(G_{0}[p]\right)$.

Displays of a $p$-divisible group over a field. In this situation we define the display of this $p$-divisible group over a field. The $\sigma$-linear map $F: M_{0} \rightarrow M_{0}$ can be written out on the $W$-basis $\left\{e_{1}=X_{1}^{\prime}, \cdots, e_{d}=X_{d}^{\prime}, e_{d+1}=Y_{1}^{\prime}, \cdots, e_{d+c}=\right.$ $\left.Y_{c}^{\prime}\right\}$ as:

$$
\begin{aligned}
F e_{j} & =\sum_{i=1}^{h} a_{i j} e_{i} & & 1 \leq j \leq d, \\
e_{j} & =V\left(\sum_{i=1}^{h} a_{i j} e_{i}\right) & & d<j \leq h=c+d ;
\end{aligned}
$$

we say that we have written the module in displayed form. We shall write

$$
\left(a_{i, j} \mid 1 \leq i, j \leq h\right)=\left(\begin{array}{cc}
A & B \\
C & D
\end{array}\right)
$$

This matrix, denoted by $(a)$, will be called the matrix of the display. Note that in this case the $\sigma$-linear map $F$ is given on this base by the matrix

$$
\left(\begin{array}{ll}
A & p B \\
C & p D
\end{array}\right),
$$

where

$$
\begin{gathered}
A=\left(a_{i j} \mid 1 \leq i, j \leq d\right), \quad B=\left(a_{i j} \mid 1 \leq i \leq d<j \leq h\right), \\
C=\left(a_{i j} \mid 1 \leq j \leq d<i \leq h,\right), \quad D=\left(a_{i j} \mid d<i, j \leq h\right) .
\end{gathered}
$$

Where the display-matrix is symbolically denoted by $(a)$, we write $(p a)$ symbolically for the associated $F$-matrix (it is clear what is meant as soon as $d$ is given).

Suppose $R$ is a complete Noetherian local ring with perfect residue class field $K$. We assume $p \cdot 1=0 \in R$. Let $t_{r, s}$ be elements in the maximal ideal of $R$, with $1 \leq r \leq d<s \leq h$, and let

$$
T_{r, s}=\left(t_{r, s}, 0, \cdots\right) \in W(R)
$$


be their Teichmüller lifts. Write

$$
T=\left(\begin{array}{ccc}
T_{1, d+1} & \cdots & T_{1, h} \\
\vdots & & \vdots \\
T_{d, d+1} & \cdots & T_{d, h}
\end{array}\right), \quad\left(\begin{array}{cc}
A+T C & p B+p T D \\
C & p D
\end{array}\right)
$$

We can ask whether this is the matrix of a display connected with a deformation of $G_{0}$. In fact:

Let $K$ be a perfect field, let $R$ be a complete Noetherian local ring with residue class field $K$, and let $G_{0}$ be a formal p-divisible group over $K$. The formulas above define a display over the ring $W$ of Witt vectors over $R$ (in the sense of [64]). Hence these formulas define a deformation $G \rightarrow \operatorname{Spec}(R)$ of $G_{0}$.

Remark. One can show that the deformation just given is the universal deformation of $G_{0}$ in equal characteristic $p$, by taking the elements $t_{r, s}$ as parameters,

$$
R:=K\left[\left[t_{r, s} \mid 1 \leq r \leq d<s \leq h\right]\right]
$$

Suppose moreover the $p$-divisible group $G_{0}$ has a principal quasi-polarization $\lambda_{0}$ and let the base $\left\{X_{1}^{\prime}, \cdots, Y_{d}^{\prime}\right\}$ be symplectic (in this case $c=h-d=d$ ).

Then assume moreover that

$$
t_{r, s}=t_{s-d, r+d} \in R
$$

the displayed form above defines a deformation $(G, \lambda)$ as quasi-polarized formal p-divisible group of $\left(G_{0}, \lambda_{0}\right)$.

In this case we renumber the elements as $x_{i, j}=t_{i, j+d}$, with $d=g=c=$ $h / 2 x_{i, j}=t_{i, j+d}$, with $d=g=c=h / 2$. In this case the formal power series ring

$$
R:=K\left[\left[x_{i, j} \mid 1 \leq i, j \leq g\right]\right] /\left(x_{i, j}-x_{j, i} \mid 1 \leq i, j \leq g\right)
$$

defines the universal deformation space of $\left(G_{0}, \lambda_{0}\right)$.

This gives an explicit description of the deformation theory of local-local $p$ divisible groups over complete local domains in characteristic $p$; also the deformation theory of such groups with a principal quasi-polarization, and hence, by the theorem of Serre and Tate, of principally polarized abelian varieties (the theory can be applied to much more general situations, but we will not need that here). 


\section{References}

[1] C.-L. Chai - Compactification of Siegel moduli schemes. London Math. Soc. Lect. Notes Ser. 107, Cambridge Univ. Press, 1985.

[2] C.-L. Chai - Every ordinary symplectic isogeny class in positive characteristic is dense in the moduli. Invent. Math. 121 (1995), 439 - 479.

[3] C.-L. Chai \& F. Oort - Density of Hecke orbits. [In preparation.]

[4] P. Deligne \& M. Rapoport - Les schémas de modules de courbes elliptiques. In: Modular functions of one variable, II (Ed. P. Deligne, W. Kuyk), Lect. Notes Math. 349, Springer - Verlag 1973; pp. 143 - 316 (pp. DeRa 1 - 174).

[5] M. Demazure - Lectures on p-divisible groups. Lect. Notes Math. 302, Springer-Verlag, 1972.

[6] J. Dieudonné - Lie groups and Lie hyperalgebras over a field of characteristic p. II. Amer. Journ. Math. 77 (1955), 218 - 244.

[7] M. Eichler - Zur Zahlentheorie der Quaternionen-Algebren. J. Reine Angew. Math. 195 (1955), 127 - 151.

[8] T. Ekedahl - On supersingular curves and abelian varieties. Math. Scand. 60 (1987), $151-178$.

[9] G. Faltings - Arithmetische Kompaktifizierung des Modulraums der abelschen Varietäten. Arbeitstagung Bonn 1984 (Ed. F. Hirzebruch et al.), Lect. Notes Math. 1111, Springer-Verlag, 1985.

[10] G. Faltings \& C.-L. Chai - Degeneration of abelian varieties. Ergebn. Math. 3. Folge, Bd. 22, Springer - Verlag, 1990.

[11] G. van der Geer - Cycles on the moduli space of abelian varieties. In: Moduli of curves and abelian varieties (the Dutch intercity seminar on moduli) (Eds C. Faber, E. Looijenga). Aspects of Math. E 33, Vieweg 1999 ; pp. $65-89$.

[12] G. van der Geer \& M. van der Vlugt - On the existence of supersingular curves of given genus. Journ. reine angew. Math. 45 (1995), 53 - 61.

[13] E. Z. Goren \& F. Oort - Stratification of Hilbert modular varieties I. Journ. Algebraic Geom. 9 (2000), 111 - 154.

[14] A. Grothendieck - Fondements de la géométrie algébrique. [Extraits du Séminaire Bourbaki 1957 - 1962.] Exp. 212, Sém. Bourbaki 13 (1960/1961): Préschémas quotients. 
[15] A. Grothendieck \& J. Dieudonné - Éléments de géométrie algébrique. Vol. II: Étude globale élémentaire de quelques classes de morphisms. Vol. III ${ }^{1}$ : Étude cohomologique des faisceaux cohérents (Première Partie). Publ. Math. 8, 11, Inst. Hautes Ét. Sc. 1961, 1961 [Cited as EGA II, EGA III]

[16] H. Hasse \& E. Witt - Zyklische unverzweigte Erweiterungskörper vom Primzahlgrade $p$ über einem algebraischen Funktionenkörper der Charakteristik p. Monatsh. Math. Phys. 43 (1963), 477 - 492.

[17] L. Illusie - Déformations de groupes de Barsotti-Tate. Exp.VI in: Séminaire sur les pinceaux arithmétiques: la conjecture de Mordell (Ed. L. Szpiro), Astérisque 127, Soc. Math. France, 1985.

[18] A. J. de Jong - Finite locally free group schemes in characteristic $p$ and Dieudonné modules. Invent. Math. 114 (1993), 89 - 137.

[19] A. J. de Jong \& F. Oort - Purity of the stratification by Newton polygons. Journ. A.M.S. 13 (2000), 209 - 241. See: http://www.ams.org/jams

[20] T. Katsura \& F. Oort - Families of supersingular abelian surfaces. Compos. Mat. 62 (1987), $107-167$.

[21] T. Katsura \& F. Oort - Supersingular abelian varieties of dimension two or three and class numbers. Algebraic Geometry, Sendai 1985 (ed. T. Oda); Adv. St. Pure Math. 10 (1987), Kinokuniya \& North-Holland, 1987.

[22] N. Katz - Serre-Tate local moduli. Exp. V $V^{\text {bis }}$ in: Surfaces algébriques, Sém. Géom. Alg. Orsay 1976-78 (Ed. J. Giraud, L. Illusie and M. Raynaud), Lect. Notes Math. 868, Springer - Verlag 1981, pp. 138 - 202.

[23] N. M. Katz - Slope filtrations of F-crystals. Journ. Géom. Algébr. de Rennes, Vol. I; Astérisque 63, Soc. Math. France, 1979; pp. 113 - 163.

[24] M. Kneser - Strong approximation. Proceed. Sympos. Pure Math., Vol. 9: Algebraic groups and discontinuous subgroups. A.M.S. 1966.

[25] M.-A. Knus - Quadratic and hermitian forms over rings. Grundl. math. Wissensch. 294, Springer - Verlag 1991.

[26] M.-A. Knus, A. Merkurjev, M. Rost \& J.-P. Tignol - The book of involutions. AMS Colloq. Publ. 44, Amer. Math. Soc. 1998.

[27] H.-P. Kraft - Kommutative algebraische p-Gruppen (mit anwendungen auf p-divisible Gruppen und abelsche Varietäten). Sonderforsch. Bereich Bonn, September 1975. Ms. 86 pp.

[28] H.-P. Kraft \& F. Oort - Finite group schemes annihilated by p. [In preparation.] 
[29] K.-Z. Li \& F. Oort - Moduli of supersingular abelian varieties. Lecture Notes Math. 1680, Springer - Verlag 1998.

[30] J. Lubin, J-P. Serre \& J. Tate - Elliptic curves and formal groups. In: Lect. Notes, Summer Inst. Algebraic Geometry, Woods Hole, July 1964; 9 pp.

[31] Yu. I. Manin - The theory of commutative formal groups over fields of finite characteristic. Usp. Math. 18 (1963), 3-90; Russ. Math. Surveys 18 (1963), $1-80$.

[32] W. Messing - The crystals associated to Barsotti-Tate groups: with applications to abelian schemes. Lect. Notes Math. 264, Springer-Verlag 1972.

[33] B. Moonen - Group schemes with additional structure and Weyl group cosets. Dept. Math. Utrecht Preprint 1134, February 2000; 35 pp. [This volume.]

[34] L. Moret-Bailly - Familles de courbes et de variétés abéliennes sur $\mathbb{P}^{1}$. Familles de courbes et de variétés abéliennes. Sém. sur les pinceaux de courbes de genre aux moins deux. Ed. L. Szpiro. Astérisque 86, Soc. Math. France, 1981; pp. 109 - 140.

[35] L. Moret-Bailly - Pinceaux de variétés abéliennnes. Astérisque 129, Soc. Math. France, 1985.

[36] D. Mumford - Constructions of deformation of Dieudonné modules. Private communication, 1968 .

[37] D. Mumford - Abelian varieties. Tata Inst. Fund. Res. \& Oxford Univ. Press, 1970 (2nd print. 1974).

[38] P. Norman - An algorithm for computing local moduli of abelian varieties. Ann. Math. 101 (1975), 499-509.

[39] P. Norman \& F. Oort - Moduli of abelian varieties. Ann. Math. 112 (1980), $413-439$.

[40] T. Oda - The first de Rham cohomology group and Dieudonné modules. Ann. Sc. Ecole Norm. Sup. 2 (1969), 63 - 135.

[41] A. Ogus - Supersingular K3 crystals. In: Journ. Géom. Algébr. de Rennes, Vol II; Astérisque 64, Soc. Math. France, 1979; pp. 3 - 86.

[42] F. Oort - Commutative group schemes. Lect. Notes Math. 15, SpringerVerlag 1966.

[43] F. Oort - Subvarieties of moduli spaces. Invent. Math. 24 (1974), 95 - 119. 
[44] F. Oort - Which abelian surfaces are products of elliptic curves? Math. Ann. 214 (1975), $35-47$.

[45] F. Oort - A stratification of moduli spaces of polarized abelian varieties in positive characteristic. In: Moduli of curves and abelian varieties (the Dutch intercity seminar on moduli) (Eds C. Faber, E. Looijenga). Aspects of Math. E 33, Vieweg 1999; pp. $47-64$.

[46] F. Oort - Newton polygons and formal groups: conjectures by Manin and Grothendieck. [To appear: Ann. Math. 152 (2000).]

[47] F. Oort -Newton polygon strata in the moduli space of abelian varieties. [This volume.]

[48] F. Oort - Foliations of moduli spaces. [In preparation.]

[49] V. Platonov \& A. Rapinchuk - Algebraic groups and number theory. Pure and appl. math. Vol. 139, Academic Press 1994

[50] M. Poletti - Differenziali esatti di prima specie su varietà abeliane. Ann. Scuola Norm. Sup. Pisa 21 (1967), 107 - 110.

[51] G. Prasad - Strong approximation for semi-simple groups over function fields. Ann. Math. 105 (1977), 553 - 572.

[52] I. Reiner - Maximal orders. Academis Press 1975.

[53] W. Scharlau - Quadratic and hermitian forms. Grundl. math. Wissensch. 270, Springer - Verlag 1985.

[54] Séminaire de géométrie algébrique, 1967-1969, SGA 7 : Groupes de monodromie en géométrie algébrique. (A. Grothendieck, M. Raynaud, D. S. Rim.) Vol. I, Lect. Notes Math. 288, Springer - Verlag, 1972.

[55] J-P. Serre - Cohomologie galoisienne. Cinquième édition, révisé et complétée. Lect. Notes Math. 5, Springer - Verlag 1994.

[56] J-P. Serre - Sur la topologie des variétés algébriques en charactéristique p. Symp. Int. Top. Alg. Mexico (1985), pp. 24-53 (see Collected works, Vol. I, pp. 501-530).

[57] G. Shimura - Arithmetic of alternating forms and quaternion hermitian forms. Journ. Math. Soc. Japan 15 (1963), 33 - 65.

[58] T. Shioda - Supersingular K3 surfaces. In: Algebraic Geometry, Copenhagen 1978 (Ed. K. Lønsted). Lect. Notes Math. 732, Springer-Verlag, 1979 ; pp. 564-591. 
[59] T. A. Springer - Linear algebraic groups. Second edition. Progress Math. 9, Birkhäuser, 1998.

[60] L. Szpiro - Propriétés numériques du faisceau dualisant relatif. Sém. sur les pinceaux de courbes de genre aux moins deux. Ed. L. Szpiro. Astérisque 86, Soc. Math. France, 1981; pp. 44-78.

[61] T. Wedhorn - The dimension of Oort strata of Shimura varieties of PELtype. [This volume.]

[62] Yu. G. Zarhin - Endomorphisms of abelian varieties and points of finite order in characteristic $p$. Math. Notes Acad. Sci. USSR 21 (1977), 734 744 .

[63] T. Zink - Cartiertheorie kommutativer formaler Gruppen. Teubner-Texte Math. 68, Teubner, Leipzig, 1984.

[64] T. Zink - The display of a formal p-divisible group, University of Bielefeld, Preprint 98-017, February 1998.

[65] T. Zink - A Dieudonné theory for p-divisible groups. Manuscript, 23 pp., September 1998.

\author{
Frans Oort \\ Mathematisch Instituut \\ Budapestlaan 6 \\ NL - 3508 TA Utrecht \\ The Netherlands \\ email: oort@math.uu.nl
}

